University of Louisville

ThinkIR: The University of Louisville's Institutional Repository

Electronic Theses and Dissertations

$12-2014$

\title{
The role of stem cells in adipose tissue remodeling.
}

Candice ReShay Holden 1988-

University of Louisville

Follow this and additional works at: https://ir.library.louisville.edu/etd

Part of the Biophysics Commons, and the Cell Biology Commons

\section{Recommended Citation}

Holden, Candice ReShay 1988-, "The role of stem cells in adipose tissue remodeling." (2014). Electronic Theses and Dissertations. Paper 1739.

https://doi.org/10.18297/etd/1739

This Doctoral Dissertation is brought to you for free and open access by ThinkIR: The University of Louisville's Institutional Repository. It has been accepted for inclusion in Electronic Theses and Dissertations by an authorized administrator of ThinkIR: The University of Louisville's Institutional Repository. This title appears here courtesy of the author, who has retained all other copyrights. For more information, please contact thinkir@louisville.edu. 


\title{
THE ROLE OF STEM CELLS IN ADIPOSE TISSUE REMODELING
}

\author{
By \\ Candice ReShay Holden \\ B.S., University of Central Florida, 2010 \\ M.S., University of Louisville, 2013 \\ A Dissertation \\ Submitted to the Faculty of the \\ School of Medicine of the University of Louisville \\ in Partial Fulfillment of the Requirements \\ for the Degree of \\ Doctor of Philosophy \\ Department of Physiology and Biophysics \\ University of Louisville, \\ Louisville, Kentucky
}

December 2014 

THE ROLE OF STEM CELLS IN ADIPOSE TISSUE REMODELING

\author{
By \\ Candice ReShay Holden \\ B.S., University of Central Florida, 2010 \\ M.S., University of Louisville, 2013
}

A Dissertation Approved on

November 25, 2014

By the following Dissertation Committee:

Bradford G. Hill, Ph.D.

Aruni Bhatnagar, Ph.D.

Irving G. Joshua, Ph.D.

Dale A. Schuschke, Ph.D.

Cynthia J. Miller, Ph.D. 


\section{ACKNOWLEDGEMENTS}

I would like to thank my mentors, Drs. Bradford Hill and Aruni Bhatnagar, for guiding me through this process and teaching me how to approach scientific problems appropriately and methodically. I would also like to thank my committee members, Drs. Irving Joshua, Cynthia Miller, and Dale Schuschke, for their advice and support during my time at the University of Louisville. I would like to acknowledge Dr. James McCracken for his patience and time which he spent helping me to learn and appreciate flow cytometry, a technique that I used in nearly all of my studies.

To my parents, Carl and Twanda Holden, you both have always encouraged me to be the best version of myself and to work hard to complete any task that is placed in front of me, no matter how daunting it may appear. To my sister and best friend, Lisa Holden, your support is undeniable and I am lucky to have you in my life. To my husband-to-be, Zaunklay Finnila, I appreciate your continued patience and kind words of encouragement. Though all of my family members have been supportive throughout my life, these four individuals have been immensely supportive and loving throughout this entire process and to them I am eternally grateful. Accomplishing this goal would have been impossible without the love and support of each of you and for that I would like to dedicate this work to all of you. 


\section{ABSTRACT \\ THE ROLE OF STEM CELLS IN ADIPOSE TISSUE REMODELING Candice ReShay Holden}

November 25, 2014

The work presented in this dissertation outlines the role of stem cells in the remodeling of adipose tissue under conditions of nutrient excess. Obesityinduced variations in adipose tissue stem cell distribution were uncovered by analysis of the stromal vascular fraction isolated from mice fed a high fat diet for several weeks. Bone marrow cell transplantation was used to determine the derivation of progenitor cells found in adipose tissue; and supplementation of depleted progenitor cell populations achieved via direct cell transplantation, helped to determine the contribution of these progenitor cells to the remodeling process.

The dissertation is presented in five chapters outlining the adipose tissue remodeling process, metabolic changes which occur in adipose tissue in response to nutrient excess, diet-induced variations in adipose tissue stem cell distribution, and the impact of stem cell transplantation on remodeling of adipose tissue. Chapter one serves as a general introduction defining the development of obesity, health implications of obesity and methods by which adipose tissue can remodel in response to nutrient excess. Chapter two describes the metabolic changes that occur within visceral adipose tissue upon consumption of high fat 
diet. The third chapter details changes in adipose tissue stem cell distribution in response to nutrient excess. The studies presented in chapter three suggest that obesity is associated with an imbalance in adipocyte and endothelial progenitor cells. This imbalance was corrected to determine if doing so was sufficient to ameliorate obesity-associated complications and data from these studies are presented in chapter four. Finally, a comprehensive discussion of the implications of these studies is outlined in chapter five.

Chapters two and three focus on the impact of high fat diet on the metabolic profile of adipose tissue and distribution of adipose tissue stem cells. Chapter four centers on correcting the progenitor cell ratio, skewed by consumption of high fat diet. The studies presented in the fourth chapter suggest increasing the endothelial progenitor cell population promotes cell engraftment into vasculature of the adipose tissue. Increasing the capacity of these cells further supports vessel formation and engraftment in obese tissue and may therefore offer insight into potential therapeutic treatments. 


\section{TABLE OF CONTENTS}

Page

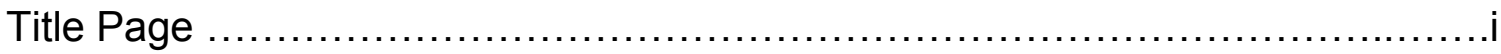

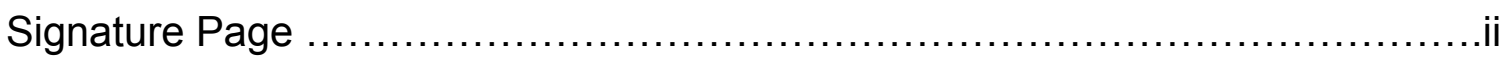

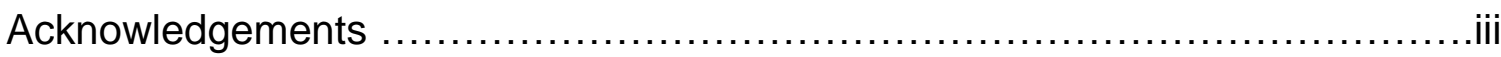

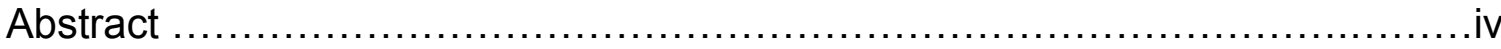

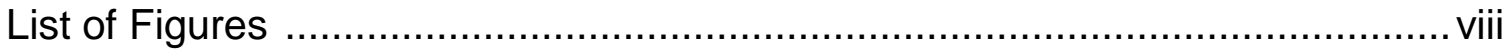

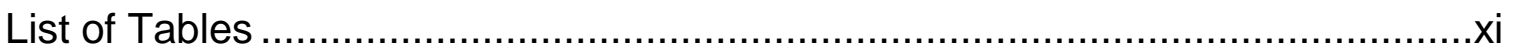

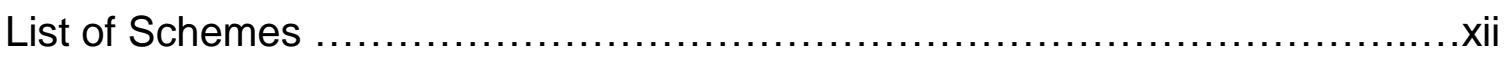

Chapter 1: General Introduction

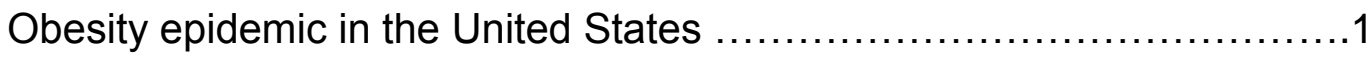

Health implications and consequences of obesity .......................11

Metabolically favorable adipose tissue remodeling .......................14

Adipose tissue stem cells ............................................ 15

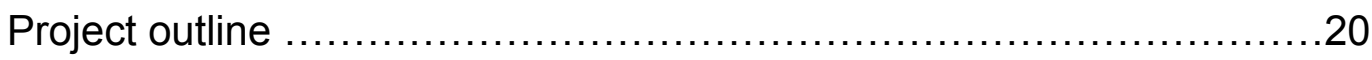

Chapter 2: Metabolic remodeling of white adipose tissue in obesity

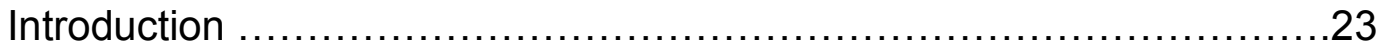

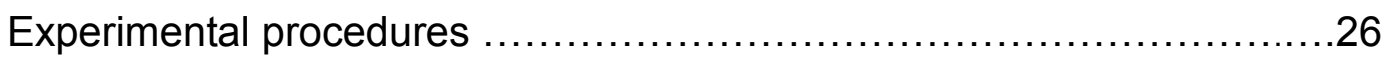

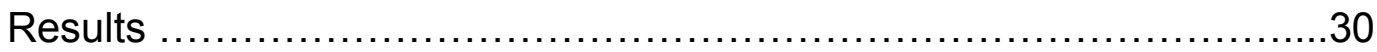

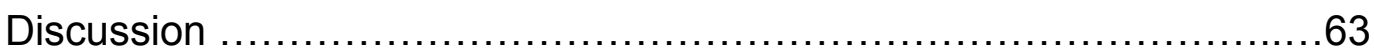

Chapter 3: Regulation of adipose tissue stem cell populations by obesity

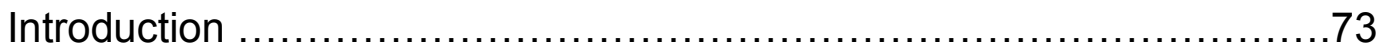

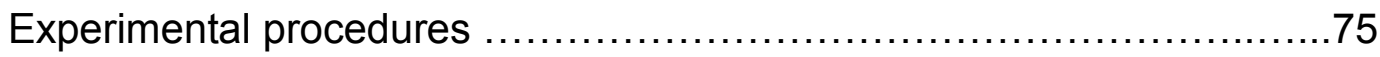

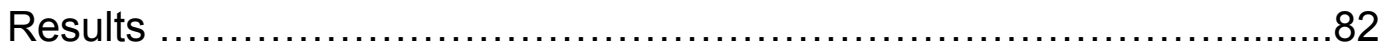

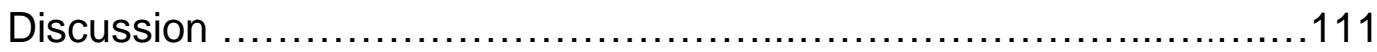


Chapter 4: Contribution of stem cells to adipose tissue remodeling

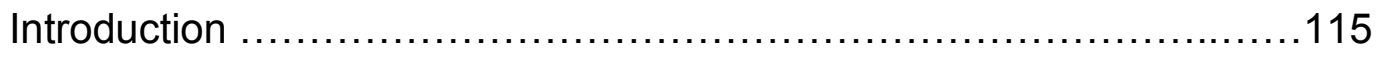

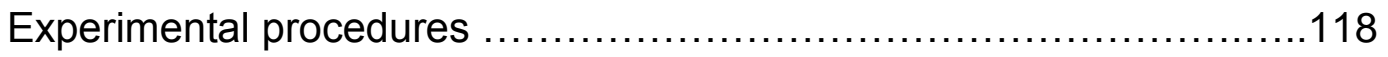

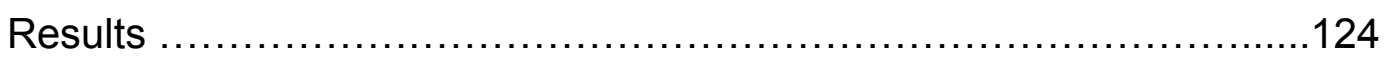

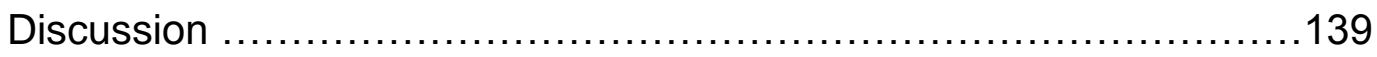

Chapter 5: Concluding Discussion .............................................

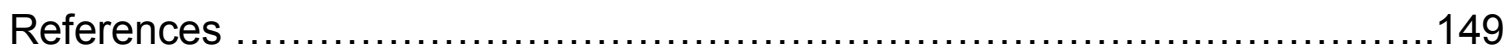

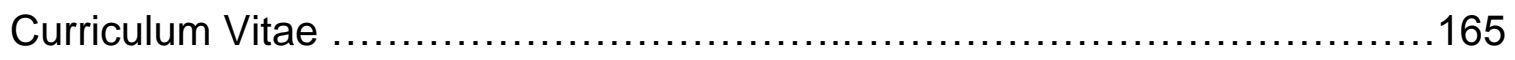




\section{LIST OF FIGURES}

Page

1: Effects of high fat diet on weight gain, adiposity and systemic metabolism ...32

2: Glucose and insulin tolerance in mice fed low fat or high fat diets .............34

3: Effect of HFD on adipose tissue expansion and inflammation ................37

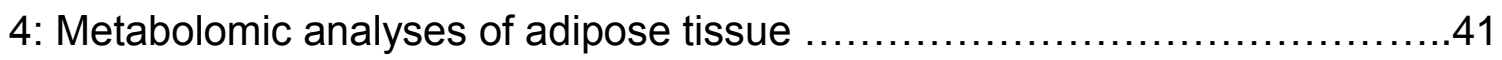

5: Z-score plot analysis of metabolite changes in adipose tissue from high fat

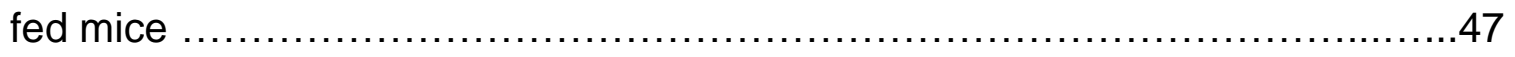

6: Illustration of metabolite changes in adipose tissues of obese mice ..........49

7: Obesity-related energetic changes in white adipose tissue ...................52

8: Obesity-related changes in mitochondrial protein abundance in white adipose

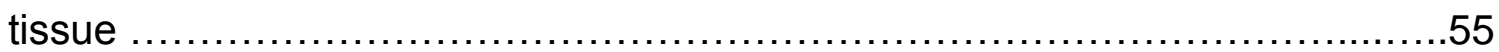

9: Ultrastructure of white adipose tissues from lean and obese mice ...........58

10: Evidence for activation of mitophagy in WAT of obese mice .................61

11: Adiposity increases significantly after consumption of a $60 \%$ fat diet for 12

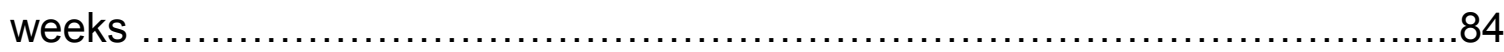

12: Consumption of high fat diet decreases physical activity $\ldots \ldots \ldots \ldots \ldots \ldots \ldots . . . . \ldots 6$

13: Measurements of insulin sensitivity in WT mice after 12 weeks of high

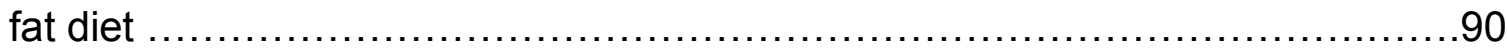

14: Wet tissue weight of organs harvested from animals fed LFD or HFD for

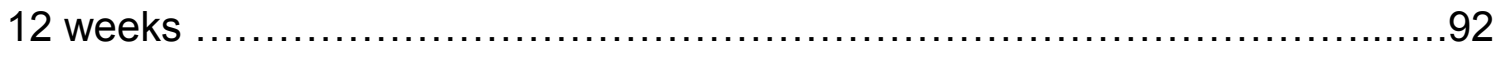


15: Quantification of F4/80+ and CD3+ cell populations in visceral and subcutaneous adipose tissue after consumption of $60 \%$ high fat diet for

12 weeks

16: Quantification of adipocyte progenitor cell, APC, and endothelial progenitor cell, EPC, populations of visceral adipose tissue after consumption of $60 \%$ high fat diet for 12 weeks 101

17: Quantification of adipocyte progenitor cell, APCs, and endothelial progenitor cell, EPC, populations of visceral adipose tissue after bone marrow repopulation and consumption of $60 \%$ high fat diet for 12 weeks 103 18: Quantification of BrdU in adipose tissue after 12 weeks if LFD or HFD .....105 19: Changes in adiposity, glucose tolerance, and insulin tolerance after reversion to $10 \%$ LFD 107

20: Quantification of adipocyte progenitor cell, APC, and endothelial progenitor cell, EPC, populations of visceral adipose tissue after consumption of $60 \%$ high fat diet for 12 weeks and reversion to $10 \%$ LFD for 4 weeks 109 21: Hematoxylin and eosin stained paraffin-embedded visceral and subcutaneous adipose tissue sections

22: Representative confocal images of isolectin stained adipose tissue sections

23: High fat diet increases adiposity in WT and eNOS-Tg bone marrow transplanted animals 130

24: Measurements of insulin sensitivity in bone marrow transplanted animals after 12 weeks of high fat diet 
25: Quantification of progenitor cell populations of the visceral adipose

tissue

26: Isolectin staining of visceral adipose tissue sections after 12 weeks of LFD or

HFD 


\section{LIST OF TABLES}

Page

1: List of adipose tissue metabolites that changed significantly in high fat-fed

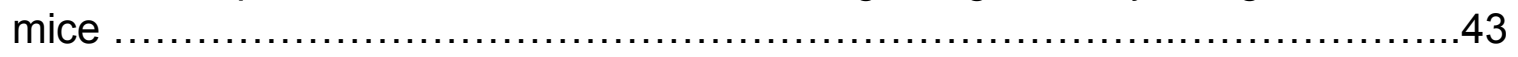




\section{LIST OF SCHEMES}

Page

1: Calculation of body mass index and body fat percentage using skin

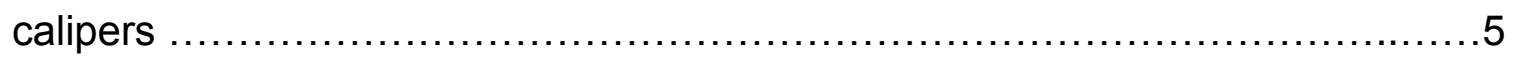

2: Bone marrow transplantation protocol ......................................... 80

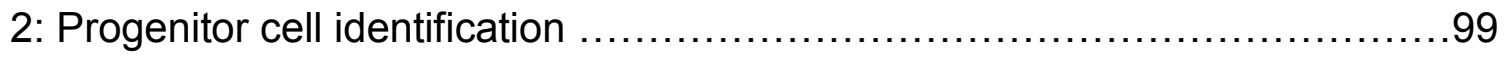




\section{CHAPTER 1}

\section{GENERAL INTRODUCTION}

\section{Obesity epidemic in the United States}

\section{Climbing rate of obesity}

The prevalence of obesity in the United States has increased remarkably over the past four decades $(1,2)$. The National Health and Nutrition Examination Survey reported the prevalence of obesity in the United States as 15\% from 1976 to 1980 (1). Between the years of 1988 and 1994, the Centers for Disease Control $(C D C)$ reported that the prevalence of obesity was approximately $22.9 \%$. By the year 2000 , the prevalence of obesity was reported to be $30.5 \%$ and, in 2010, that statistic increased again to $35.7 \%(3-6)$. It was recently reported that, in the United States, 34.9\% of individuals over the age of 20 are currently obese (7). In the state of Kentucky, $31.3 \%$ of the population is considered obese (4). The high prevalence of obesity is important because it increases the incidence of pre-diabetic and diabetic states and is associated with numerous co-morbidities. As such, a substantial financial burden, due to obesity, has been imparted on the economy of the United States. Most recent estimates suggest that $\$ 147$ billion US dollars are spent on the treatment of obesity with obese individuals paying an additional $\$ 1,429$ each year for medical treatment (8)! 
Potential causes for this relatively recent increase in obesity prevalence are low levels of physical activity and increased caloric intake. The CDC reports that only $48 \%$ of adults meet the guidelines for physical activity, i.e., 30 minutes of moderate activity or endurance training 5 days per week or 20 minutes of vigorous activity 3 days per week $(9,10)$. In Kentucky, where the obesity prevalence is above $30 \%$, only $17.7 \%$ of the state's surveyed population were in compliance with the CDC's physical activity recommendations in $2000(4,11)$. In 2003-2004, the CDC sampled 1,714 adults and reported that only $3 \%$ of nonsleeping time was spent exercising, $58 \%$ of the day was spent in sedentary behavior and 39\% was spent engaging in a light-intensity activity (12).

Hyperphagia and consumption of a diet rich in fat, are also known to contribute to the development of obesity (13-15), and, expectedly, physical inactivity combined with the consumption of excess nutrients exacerbates the obese condition (11). Excess lipids are deposited in peripheral organs and this ectopic deposition of fat has been implicated in the development of several chronic disease states (16). Ectopic fat deposition in organs such as the skeletal muscle, pancreas, and liver has been shown to result in glucose intolerance, hyperinsulinemia, and hyperlipidemia $(16,17)$. Though diet-induced obesity is the predominant focus of our work, there is also a wealth of evidence to suggest that genetic makeup could also play a role in the determination of body composition (13, 18-21). 
Definition and Diagnosis of obesity

Obesity develops as caloric intake consistently exceeds energy expenditure generating a surplus of nutrients which are not used for biological processes and are stored as esterified fat. This condition has been classically defined as having a body mass index $(\mathrm{BMI}) \geq 30$. With the rate of obesity steadily rising, in November of 2013, the American Medical Association suggested that obesity should be acknowledged as a disease and should be treated as such. This would include physicians advising patients to utilize weight loss programs as a means to avoid obesity-related complications and to improve and potentially alleviate any pre-existing conditions related to increased adiposity (22).

BMI is calculated using body weight and height (Scheme 1). It provides an estimate of the body fat content of an individual. Using a BMI measurement as the sole method for quantification of body fat content could lead to an inaccurate estimation for exceptionally tall individuals over 7 feet in height, those with decreased muscle mass such as the elderly, those with increased muscle mass such as athletes, and pregnant woman. To more accurately assess body fat, multiple measurements should be made.

There are several ways to measure body fat, including measurement of waist circumference, skin calipers, bioelectrical impedance, and Dual-energy Xray Absorptiometry or Dexascan. Measurements of waist circumference and bioelectrical impedance are relatively simple to obtain. According to the National Institutes of Health $(\mathrm{NIH})$, women with a waist circumference $\geq 35$ inches or men measuring $\geq 40$ inches show increased risk for obesity-related diseases. 
Bioelectrical impedance utilizes gentle electrical impulses and the difference in conductivity of fat and muscle to discriminate between lean mass and fat mass. Calipers can be used be used to measure body fat. For this, the skin is pinched in several locations and the thickness of the skin flap is measured. Calipers can be used on the arm, abdominal region, thighs, and several other locations. The measurements from each of these regions along with the individual's age can then be used in an algorithm to calculate body fat. This algorithm utilizes the Jackson-Pollack and Siri equations as described in Scheme 1 (23-25). Tools to complete all of these assessments are readily available.

Dexascan, the most accurate and costly of the techniques presented here, takes very little time to complete and is painless to the subject, however it cannot be measured at home. For this, the subject undergoes a fairly standard X-ray procedure and the bone, fat, and lean mass can be calculated based upon the absorption of $x$-rays in each tissue type (26). 


\section{Scheme 1}

A.

Calculation of body mass index:

$\mathrm{BMI}=\underline{\text { Weight }(\mathrm{lbs}) \times 703}$

(Height (in))

B.

Calculation of body density: Jackson-Pollock formula

Males:

Body Density $=[1.10938-0.0008267 \times($ chest + abdomen + thigh $)]+\left[0.0000016 \times(\text { chest }+ \text { abdomen }+ \text { thigh })^{2}\right]-(0.0002574 \times$ age $)$

Females:

Body Density $=[1.0994921-0.0009929 \times($ triceps + waist + thigh $)]+\left[0.0000023 \times(\text { triceps }+ \text { waist }+ \text { thigh })^{2}\right]-(0.0001392 \times$ age $)$

Calculation of body fat percentage: Siri equation

$\%$ Body Fat $=100 \times\left(\frac{4.95}{\text { body density }}-4.5\right)$

* Please note that all skin thickness measurements should converted to millimeters. 
Scheme 1. Calculation of body mass index and body fat percentage using skin calipers. The equation for the calculation requires a measurement of total body weight and height as outlined above, (A). Total body density is first calculated using the Jackson-Pollack equation which is gender specific. The Siri equation, which can be applied to both males and females, is then used to determine the body fat percentage, (B). 
Function and types of adipose tissue

It has been suggested that adipose tissue is a major regulator of metabolic homeostasis. This is partly via its chief function, which is to esterify free fatty acids (FFA) and store excess lipids as triglycerides to serve as a reserve energy source (16). The ability to compile a nutrient reserve from excess caloric intake is conserved across several species $(21,27,28)$. In humans, fat is stored in visceral and subcutaneous white adipose tissue depots which are located in several areas of the body. Visceral depots are most often found in the abdominal region, surrounding the intestines and kidneys. Subcutaneous depots are located just beneath the skin and pads in the abdominal area, buttocks, and thighs are most substantially expanded due to nutrient overload. Distribution of fat changes with age, typically increasing more in the visceral abdominal region (27). Insulin resistance occurs when fat can no longer be accommodated by these depots and begins to accumulate in non-adipose organs such as skeletal muscle, liver, kidneys, and the heart (16).

Though white adipose tissue is the most abundant fat source in adults, brown and beige adipose tissues also function to maintain metabolic stability. Brown fat is also believed to originate from the mesoderm and is a highly active tissue (27). The primary function of brown adipose tissue is thermogenesis. In newborns there is an increased amount of brown fat that is significantly decreased with age. This tissue helps to maintain basal body temperature and can be stimulated to increase energy expenditure and produce heat in times of need with the support of uncoupling protein-1 (UCP-1) located in the 
mitochondrial membrane (29). In conditions where brown adipose tissue is not present, diet-induced obesity, diabetes, and hyperlipidemia have been observed (30).

White adipose tissue has been shown to dedifferentiate and change to a more "brown-like" phenotype due to extended exposure to cold or increased sympathetic stimulation (27, 31-33). UCP-1 positive cells appear to be dispersed throughout white adipose depots. More recently, these cells have been called "beige" adipocytes. These cells have a lineage consistent with that of white adipocytes but express UCP-1 and generate heat upon stimulation $(17,23-26)$. UCP-1 expression has also been observed to increase with the use of thiazolidinediones (TZDs) (34).

\section{Adipose tissue composition and progenitor identification}

Adipose tissue is composed of a multitude of cells, the functional cell type being adipocytes. Mature or terminally differentiated adipocytes can easily be identified by immunohistological staining for the protein perilipin. Perilipin is found in the thin cell membrane of the adipocyte. Oil Red-O and Nile red dyes can be used to identify lipid droplets within the mature adipocytes. In white adipose tissue, these droplets are large and unilocular, unlike those of brown adipose tissue which are multilocular in nature. As previously described, brown adipocytes express UCP-1 whereas white adipocytes are leptin positive. Both white and brown mature adipocytes express Peroxisome Proliferator- Activated Receptor gamma 2 (PPARY2), Glucose Transporter Type 4 (GLUT4), fatty acid synthase, and are regulated by insulin $(27,35)$. 
When adipose tissue is digested using collagenase to isolate specific cell types of interest, lipid-loaded adipocytes float due to decreased density. The mixture of cells which can be pelleted is referred to as the stromal vascular fraction (SVF). The SVF contains mesenchymal stem cells including preadipocytes, vascular/endothelial progenitor cells, pericytes, dendritic cells, fibroblasts, mature endothelial cells, immune cells such as macrophages, T cells, B cells, and monocytes as well as a host of other cell types (36). Preadipocytes or adipocyte progenitor cells can be distinguished by the expression of CD29, CD24, CD34, and stem cell antigen-1 (Sca-1).

In 2008, Rodeheffer, et al. showed that progenitor cells were sorted from the SVF based upon their expression profile of cell surface antigens for CD24, CD29, CD34, CD31, Sca-1 and lineage marker (Lin). Four cell types were isolated and cultured for in vitro analysis. Cultures enriched for cells positive for CD29, CD34, and CD24 and negative for CD31 and the lineage marker (Lin) showed a significant increase in Oil Red-O staining compared to unfractionated SVF. However, when expression levels of adipsin and aP2 were quantified, cells which were positive for CD24 in addition to CD29, CD34, and Sca-1 and negative for CD31 and the lineage marker (Lin) had the highest levels of expression for both adipocyte markers. When CD24, CD29, CD34, Sca-1 positive and CD31 and Lin negative cells were transplanted into insulin resistant A-Zip mice lacking functional epididymal white adipose tissue, the cells were able to proliferate and form a functional fad pad similar in size to that of a normal, wild-type mouse just 12 weeks after injection of 50, 000 cells. Systemically, plasma insulin and 
glucose levels decreased to the level of the wild-type and adiponectin levels were increased. The cells were harvested from mice expressing green fluorescent protein (GFP) and after completion of the study, the fat pads harvested from the transplanted A-Zip mice were identified as GFP positive, unilocular, lipid-loaded cells (37).

Adipose tissue deficiency: Lipodystrophies

Although more than one-third of the US population struggles with obesity, a smaller amount of Americans, less than 200,000 people, have the opposite problem and are unable to store fat properly. This is a rare condition described by the NIH-Office of Rare Diseases as lipodystrophy. This disease can be attributed to a marked decrease in leptin, utilization of certain drug therapies such as antiretroviral drugs which are commonly used for the treatment of HIV, or genetic predisposition (38).

Several types of lipodystrophy can be attributed to genetics $(39,40)$. Mutations in genes encoding for PPARY, AGPAT2, AKT2, CAV1, PTRF, CIDEC, lamin A/C, ZMPSTE24, and BSCL2 are some of the causes for the development lipodystrophy. These mutations can cause problems in the development, differentiation, or lead to the premature death of adipocytes. Due to the inability to properly store fat, individuals with lipodystrophy are predisposed to diabetes, pancreatitis, end stage renal disease, diabetic retinopathy, CHD, renal failure, and even arrhythmias which could induce sudden death. Currently, the only approved treatments for individuals with this disease work to decrease excess visceral adiposity. There is not much that is currently offered to alter fat storage 
in lipodystrophy individuals, such a treatment might create a more favorable, less pathogenic distribution of fat (40).

\section{Health implications and consequences of obesity}

Insufficient amount of physical activity and increased in caloric intake are major contributors to the rise of obesity in American adults. The development of obesity is associated with several complications. Obesity- associated complications include, but are not limited to: insulin resistance, Type 2 Diabetes (T2D), predisposition for certain cancers, hypertension, dyslipidemia, metabolic syndrome, stroke, and coronary heart disease (10, 41-46).

Coronary heart disease $(\mathrm{CHD})$ is defined as the development of plaques in coronary arteries which may impede blood flow as they become larger over time. This decrease in blood supply could lead to angina, heart attack and arrhythmias. There is evidence that increasing aerobic activity beyond the amount specified in guidelines set by the American Heart Association (AHA) and American College of Sports Medicine has potential health benefits and can reduce the risk for CHD (10). The National Runners' Health Study provided information from a cohort of 8,283 runners in which the amount of self-reported weekly physical activity was strongly correlated with biomarkers of physical activity such as increased high-density lipoprotein (HDL), reduction of hypertension, lower blood pressure, decreased adiposity, lower triglyceride level, and decrease CHD risk (41). After 10 years, a follow-up survey was administered 
to the group inquiring about the need for or completion of coronary artery bypass surgery and percutaneous transluminal coronary angioplasty as well as information about the number of heart attack and angina diagnoses experienced by the study participants. This assessment revealed the correlation of running and the reduction of $\mathrm{CHD}$ risk could be mediated by the baseline BMI of the individual, suggesting that running may be of greater benefit to individuals with a higher BMI than those with a lower BMI at baseline. However, higher baseline BMI significantly increased the risk for the development of $\mathrm{CHD}$, hypertension, high cholesterol, and diabetes; even in those that engaged in vigorous physical actively on a regular basis (10).

There have been numerous studies implicating obesity as a confounding factor for the development of Type 2 Diabetes (T2D) and insulin resistance (4246). The underlying cause being adipocyte dysfunction and increased caloric intake for both T2D and insulin resistance offers support for the association between the conditions and the development of obesity. It has also been established that adipose tissue is a major regulator of systemic glucose and lipid homeostasis.

Obesity can induce both changes in metabolism within adipocytes themselves and more broad changes in the adipose tissue as whole. Diet induced-obesity has been shown to cause a decline in mitochondrial abundance in white adipocytes over time (47-50). This phenomenon has been described as adipose tissue "whitening" $(15,51,52)$. The dynamic nature of adipose tissue allows adipocytes to change phenotype along a continuum with highly 
metabolically active brown adipocytes at one end and white adipocytes with decreased metabolic activity at the other $(15,53)$. Metabolic changes that occur during the whitening process have not been completely described; however, our metabolomic analyses have revealed that diet-induced metabolic changes precede adipose tissue inflammation and loss of mitochondria in visceral adipose tissue. These changes appear to coincide with perturbations of lipid, amino acid, glucose, and energy metabolism, posing a threat to systemic metabolic homeostasis (15).

As previous stated, consumption of HF diet can also cause dysfunction in adipose tissue as a whole. Under conditions of nutrient excess, some adipocytes expand to sizes as large as $10,000 \mu m^{2}(54)$. Such expansion requires extreme remodeling of the adipose tissue microenvironment. Adipocyte expansion at that level can lead to hypoxia due to the inability of oxygen to diffuse such a great distance from the capillary and cell necrosis once the adipocytes hypertrophy pass the point of sustainability (55-58). Hypertrophied adipocytes can secrete a host of pro-inflammatory cytokines and chemokines including macrophage chemoattractant protein (MCP-1). This results in the recruitment of inflammatory cells to clear dead cells $(58,59)$. Influx of inflammatory cells like macrophages and $T$ cells, creates a state of low-grade chronic inflammation. Tumor necrosis factor- $\alpha(T N F \alpha)$ is also increased in inflamed adipose tissue spurring more recruitment of inflammatory cells and disrupting the lipid storage process leading to insulin resistance. Together, these events are indicative of unfavorable, pathological adipose tissue remodeling $(42,56,60,61)$. 


\section{Metabolically favorable adipose tissue remodeling}

A major remodeling of the adipose tissue is believed to occur after resolution of the necrosis-induced inflammatory response associated with excessive adipocyte hypertrophy. This phenomenon has been coined the "necrotic wave". After resolution of inflammation, progenitor cells in the adipose tissue proliferate, differentiate, and repopulate the organ. This is associated with restoration of insulin sensitivity and is known as favorable adipose tissue remodeling (56).

Increased storage capacity of the white adipose tissue depots appears to be beneficial to systemic metabolism. An increase in fat storage capacity decreases ectopic storage of excess lipids in peripheral organs, which appears to prevent insulin resistance. One such example, was reported by Dr. Philipp Scherer's group. They studied transgenic mice overexpressing adiponectin. The mice presented a phenotype of healthy, morbid obesity. The group proposed that the increase in adiponectin served as somewhat of a starvation signal, prompting the body to store esterified fat in the adipose tissue as opposed to peripheral organs. In so doing, there was no substantial ectopic deposition of fat which allowed for the preservation of insulin sensitivity and metabolic homeostasis in the transgenic mice (41).

Likewise, obese $(o b / o b)$ mice expressing a mitochondrial membrane protein called MitoNEET have the capacity to store enormous amounts of fat while still maintaining systemic insulin senstivity and preventing adipose tissue inflammation. MitoNEET exists in the outer mitochondrial membrane and 
functions to increase the overall activity of the mitochondria, stimulate adiponectin production, and decrease damage due to reactive oxygen species. Mice overexpressing this gene can therefore store a tremendous amount of lipid in their white adipose tissue depots (62). In both cases, the mice become morbidly obese with body weights as high as 130 grams, yet metabolic homeostasis was not disturbed.

In both metabolically normal, obese transgenic mouse models, the adipose tissue consists of numerous small adipocytes which have been shown to derive from adipocyte precursor cells $(37,62,63)$. The presence of smaller adipocytes have been shown to correlate with increased lipid storage capacity and increased mitochondrial density promoting metabolically favorable adipose tissue remodeling (64). Perhaps availability of adipocyte precursors with increased proliferative and differentiation capacity could be important in promoting favorable metabolic remodeling under conditions of nutrient excess.

\section{Adipose tissue stem cells}

\section{Origin and regulation}

Fat is believed to have originated from the mesoderm in humans, similar to bone and muscle tissues. Mesenchymal stem cells (MSCs), found in bone marrow, also have mesodermal lineage and upon differentiation, these cells have the capacity to differentiate into adipocytes, osteoblasts, chondrocytes, myoblasts, and connective tissue (27). Cells that give rise to mature adipocytes 
are known as preadipocytes or adipocyte progenitor cells. Though the specific number of steps in the differentiation process of a MSC to a mature adipocyte is unclear, Spiegelman and Rosen propose that there are four distinct stages in the maturation process of murine preadipocytes. These stages are growth arrest, clonal expansion, early differentiation, and terminal differentiation (65). Each of these steps can be transcriptional regulated impacting differentiation and maturation of the adipocyte. Major contributors to these processes are PPARY and C/EBP.

As previously discussed, mesenchymal stem cells expressing CD24, CD29, CD34, and Sca-1 can give rise to mature adipocytes in vivo, but these cells have been shown to lose expression of CD24 after commitment to the adipocyte lineage. It was reported that 4 days after transplantation of CD24 positive preadipocytes into A-Zip mice, $91.4 \%$ of the transplanted cells were negative for CD24 after analysis by flow cytometry $(37,66)$. This could prove to be an issue in tracking these newly modified cells. Interestingly, the CD24 negative cells showed increased expression levels of adipocyte markers like PPARY and C/EBPa.

PPARY has been shown to have the most profound effect on adipocyte differentiation. Without adequate expression of the transcription factor, cells will not terminally differentiate and in some cases, may even dedifferentiate, resulting in lipid unloading and a decline in adipocyte marker expression. There are two PPARY isoforms, PPARY1 and PPARY2. PPARY2 is an accepted adipocytespecific marker, though in cases when PPARY2 is unavailable, PPARY1 has 
been shown to be unregulated in an attempt to maintain metabolic homeostasis. In mice without the gene for PPARY2, adipogenesis is decreased as well as insulin sensitivity $(27,35)$. C/EBP is a family of transcription factors including CHOP, C/EBPa, C/EBP $\beta$, C/EBPD, and C/EBPY; C/EBPa having the most profound impact on adipogenesis in white adipose tissue (27). Without C/EBPa, mice developed hypertrophied brown adipocytes and were unable to form mature white adipose tissue in both subcutaneous and visceral depots (67).

Specific cells of interest

Our work has been focused on the role of adipose progenitor and endothelial progenitor cells. Both cell types have been shown to have regenerative capacity, improving peripheral blood flow $(68,69)$ and repopulating the adipose tissue during the remodeling process (56). As previously stated, mesenchymal stem cells of the adipose tissue commit to the adipocyte lineage and are then named adipocyte progenitors or preadipocytes. The commitment of these cells is transcriptionally regulated in large part by PPARY. Once the cells have committed, adipogenesis commences and the cells begin to take up lipid. The lipid-loaded cells are then considered to be mature adipocytes (70).

Endothelial progenitor cells (EPCs) have been shown to integrate into existing vasculature and improve blood flow. These cells are recruited to sites of vascular injury to help repair damage. Levels of these cells can be influenced not only by obesity, but by several environmental factors such as exposure to air pollution and components of cigarette smoke $(68,69,71,72)$. Reduced EPC count has been correlated with increased risk of cardiovascular disease and 
decreased ability to create functional vascular networks, thus these cells are of particular interest to our group (73-78). The cells were first characterized by a group at Tuft's University lead by Takayuki Asahara. In 1997, this work was published in Science and is now considered to be a seminal paper in the field of vascular biology. The paper asserts that progenitor cells in blood have the capacity to improve vascularity in ischemic tissues. These cells have been shown to differentiate into mature endothelial cells in vivo and integrate into damaged vascular beds or organize to form new capillary networks. CD34+ cells isolated from human blood and Flk1+ cells isolated from mouse blood were transplanted into a mouse or rabbit model of hindlimb ischemia 2 days after removal of the femoral artery. CD34+ cells integrated into damaged capillary networks and generated new capillary beds in the ischemic limb within 6 weeks of transplantation. Flk+ cells were also observed in capillaries and small arteries in the ischemic limb 4 weeks after transplantation. This integration was not observed in non-ischemic limbs suggesting EPCs promote vascularity in ischemic tissue. All of the transplanted cells which engrafted into the ischemic tissues stained positive for CD31, a marker of mature endothelial cells, corroborating the group's hypothesis that EPCs differentiate into mature endothelium in vivo (79).

Since the initial identification of EPCs, several studies have confirmed that progenitors similar to Asahara's, CD34+ or Flk+ cells have regenerative capacity (68, 69, 71, 80-82). Taken together, it can be concluded that EPCs function to repair damaged vasculature in order to maintain adequate tissue oxygenation, 
preventing further ischemia-induced cell death and fibrosis (75). Interestingly, the term "EPC" is a general one describing a variety of cell types that have been shown to promote angiogenesis or vessel repair $(68,69,71,75,80,82)$. We chose to investigate the role of Flk1 and Sca-1 positive cells which have been affirmed as an EPC population in mice $(68,69,71)$. We hypothesize that both adipocyte and endothelial progenitor cell populations are crucial for the favorable remodeling of adipose tissue during obesity, ameliorating hypoxia and promoting adipocyte turnover. 


\section{Project Objective}

Obesity is one of most rapidly growing health crises in the United States and its prevalence is on the rise worldwide. Increased risk for the development of Type 2 diabetes, insulin resistance, and cardiovascular disease are just three of the most devastating complications associated with obesity. Achieving a more complete understanding of the mechanisms that regulate adiposity could facilitate the development of new treatment strategies for controlling obesity and the associated cardiovascular complications. Environmental and genetic factors can contribute to adipose tissue distribution and increased adiposity, but studies investigating a form of "healthy," morbid obesity could provide insight into the mechanism by which lipid storage can be stimulated under conditions of nutrient excess while maintaining metabolic homeostasis, preventing diet-induced insulin resistance. Small adipocytes, lack of adipose tissue inflammation, and adequate adipose tissue oxygenation are hallmarks of this favorable remodeling process, which is in stark contrast to typical unfavorable, diet-induced pathological adipose tissue remodeling, characterized by large adipocytes, adipose tissue hypoxia, chronic inflammation, and systemic insulin resistance. We are interested in determining why the pathological form of adipose remodeling is generally the default for lipid storage. We hypothesize that obesity impairs stem cellmediated adipose tissue remodeling.

Although obesity is a disease relating to metabolism in general, little is known about the metabolic changes occurring in adipose tissue during the course of obesity. This is important because of the likely metabolic 
underpinnings of pathological and metabolically favorable adipose tissue remodeling. Thus, in Chapter 2, we examine the metabolic changes that occur in the visceral adipose tissue with obesity. Importantly, this involves obesity model characterization and in-depth assessment of the metabolic pathways that change in pathological forms of adipose remodeling. To determine how obesity regulates stem cell populations in adipose tissue, we assessed changes in stromal adipose stem cell populations in visceral and subcutaneous adipose tissue of adult C57BL/6J mice fed a low- or high-fat diet (Chapter 2). To completely address Aim 1, we also measured proliferation of adipose tissue stem cells in obese and lean mice, and examined how reversion to a low-fat diet affects adipose tissue stromal cell population in mice. The results from these studies will be discussed in Chapter 3. Our data suggest that conditions of obesity result in a significant decline in the availability of endothelial progenitor cells in the adipose tissue. To delineate the contribution of stem cells to adipose tissue remodeling, isolated endothelial progenitor cells were also transplanted directly into the visceral adipose tissue to determine if supplementation of these vascular progenitors was sufficient to promote a positive form of adipose tissue remodeling (Chapter 4 , Aim 2). Since we also saw a decrease in endothelial nitric oxide synthase with increased adiposity (54), bone marrow cells were isolated from mice overexpressing endothelial nitric oxide synthase and transplanted into wild-type mice which were then placed on high-fat diet for several weeks. The hypothesis being that simultaneously increasing endothelial nitric oxide synthase and stem cells availability in the adipose tissue may be sufficient to promote physiological 
adipose tissue remodeling. Changes in body weight, insulin sensitivity, inflammation, adipocyte hypertrophy, were measured. The results of these studies will help in assessing the impact of obesity and inflammation on the competency of adipose tissue stem cells and in evaluating whether cell therapy could improve metabolic dysregulation associated with obesity. 


\section{CHAPTER 2 \\ METABOLIC REMODELING OF WHITE ADIPOSE TISSUE IN OBESITY}

\section{Introduction}

The increasing prevalence of obesity is a principal health concern worldwide. In 2008, approximately 1.5 billion adults aged 20 years or older were overweight, and $10 \%$ were obese (83). In the US, more than one-third of the adult population is currently obese (BMI $>30)$, and $68 \%$ have a $\mathrm{BMI}>25(84)$. By the year 2025, these numbers are expected to increase by more than $50 \%(85)$. These statistics are a cause for alarm. Obesity is a powerful predictor of insulin resistance (86) and a major risk factor for several common medical conditions, such as type 2 diabetes (T2D), cardiovascular disease, non-alcoholic fatty liver and gallstones, Alzheimer's disease, and cancers such as breast, colon, endometrial, and esophageal cancer (87).

While lack of exercise is an undeniable risk factor for weight gain (87-89), excessive caloric intake appears to be one of the key factors fueling the obesity epidemic. In the past three decades, the average consumption of calories in the US has increased by at least $200 \mathrm{kcal} / \mathrm{d}$ per person, which could be partly attributable to an increase in the intake of energy-dense foods (90-93). Such poor dietary habits negatively affect metabolic homeostasis, which could not only promote obesity, but hasten the development of obesity-related co-morbidities as 
well. Despite the simplicity of the apparent remedy (i.e., decreasing caloric intake), treatment of obesity remains a challenging crisis facing the health care system. The efficacy of losing weight via caloric restriction is limited by multiple challenges. These include an evolutionarily engendered guard against starvation and low fat mass $(85,94)$ and a propensity to increase caloric efficiency during dieting $(95,96)$. While drugs with anorectic and anti-obesogenic properties are in clinical use, many of these show marginal long-term efficacy or have unacceptable or overtly dangerous side effects. Thus, recent strategies to modulate obesity have begun to target tissues that naturally regulate energy metabolism (85).

Increasing energy expenditure by modulating adipose tissue activity could be an attractive target for therapy. Because adult humans maintain small depots of brown fat capable of burning significant amounts of calorific energy (97-100), multiple studies have focused on the physiological and molecular mechanisms regulating the thermogenic capacity of adipose tissue. These studies have shown that adaptive thermogenesis in brown fat can be a powerful regulator of systemic energy metabolism. However, the relatively small amount of brown adipose $(<0.4 \%$ of body weight) compared with white adipose tissue (WAT; which can comprise $>40 \%$ of the body weight of an obese human) suggests that WAT may be a more tangible target. Interestingly, white adipose depots, which typically function to esterify free fatty acids and store excess lipids, have the capacity to develop into brown adipose-like tissue capable of modulating systemic metabolism and preventing obesity and insulin resistance (101). 
While the phenomenon of adipose tissue "browning" is an active area of research, there is also considerable interest in understanding the metabolic changes that occur in WAT with obesity. It is becoming increasingly clear that conditions of nutrient excess promote "whitening" of adipose tissue characterized by a decrease in mitochondrial abundance (47-49). Hence, while promoting browning is one way to positively modulate metabolism, decreasing adipose tissue whitening could be another strategy to prevent dysregulation of systemic metabolism during obesity. Indeed, the beneficial metabolic changes induced by drugs such as rosiglitazone and pioglitazone have been suggested to be due, in part, to their ability to prevent loss of mitochondria or increase mitochondrial function in WAT $(49,102)$. Nevertheless, mitochondrial changes in WAT during the development of obesity have not been thoroughly examined, and it remains unclear how changes in adipocyte metabolism affect adipocyte hypertrophy and metabolic homeostasis.

In this study, we examined WAT-specific changes in metabolism in a mouse model of diet-induced obesity. Our data indicate extensive metabolic remodeling of WAT that precedes both the infiltration of inflammatory cells and overt decreases in mitochondrial abundance. This renovation of adipocyte metabolism involved changes in glucose and lipid pathways that appear to favor fat storage and prevent excessive lipid oxidation. The metabolite profile also yields evidence of osmotic stress and adipocyte inflammation. Restructuring of intermediary metabolism was accompanied by biochemical and ultrastructural evidence of autophagy, which may promote degradation of mitochondria. These 
findings have important implications for understanding the metabolic effects of obesity on adipose tissue metabolism and suggest that targeting metabolic pathways that contribute to adipose tissue whitening or hypertrophy could form the basis for novel therapies to combat metabolic disease.

\section{Experimental procedures}

Animal studies: All procedures were approved by the University of Louisville Institutional Animal Care and Use Committee. C57BL/6J (wild-type; WT) mice were purchased from The Jackson Laboratory (Bar Harbor, ME). At 8 weeks of age, male mice were placed on either a 10\% low fat diet (LFD; Research Diets, Inc., \#D12450B) or a 60\% high fat diet (HFD; Research Diets Inc., \#D12492) for 6 or 12 weeks. Water and diet were provided ad libitum. Body weights were recorded weekly.

Metabolic phenotyping: Body composition was measured by dual-energy X-ray absorptiometry using a mouse densitometer (PIXImus2; Lunar, Madison, WI), and whole body energy expenditure; respiratory exchange ratio; food consumption; and locomotion, ambulatory and fine movements were measured using a physiological/metabolic cage system (TSE PhenoMaster System, Bad Homberg, Germany) (103). Glucose and insulin tolerance tests and plasma levels of insulin were measured exactly as described in Sansbury et al (103).

Adipocyte size measurements: Adipose tissue was excised at the time of euthanasia, and wet weight was recorded. All adipose tissue was either snap- 
frozen at $-80^{\circ} \mathrm{C}$ or fixed in $10 \%$ formalin, paraffin embedded, and sectioned. The sections were stained in hematoxylin and eosin. Adipocyte cross-sectional area and distribution was determined using Nikon Elements software. Adipose tissue sections were assessed for crown-like structures as described previously (104).

Adipose tissue metabolite profiling: WAT from the epididymal fat pads of mice fed LFD or HFD for 6 weeks were used for these analyses. Prior to tissue collection, the mice were fasted for $16 \mathrm{~h}$. After euthanasia, the adipose tissue was removed and immediately snap-frozen in liquid nitrogen. Relative metabolite abundance was then measured by GC/MS or LC/MS as described before $(103,105)$. Metabolites with missing values were imputed by replacing missing values with half of the minimum positive value in the original data. After a generalized logarithm transformation, the data were autoscaled, i.e., mean-centered and divided by the standard deviation of each variable. This step was performed to transform the intensity values so that the distribution was more Gaussian. Univariate (e.g., volcano plots), multivariate (e.g., PLS-DA), and cluster (heatmap and dendogram) analyses were then performed. Most analyses were performed using Metaboanalyst 2.0 software (http://www.metaboanalyst.ca/) (106); z-score plots were constructed in GraphPad 5.0 software.

Adipose tissue bioenergetic measurements: The oxygen consumption rate (OCR) of intact WAT explants was measured using a Seahorse XF24 analyzer (Seahorse Bioscience, Billerica, MA) as described previously (103). At least two replicates from each animal were used for the assay. After baseline measurements, the maximal OCR was measured by exposing the explants to 
FCCP $(10 \mu \mathrm{M})$. The non-mitochondrial OCR was measured following injection of antimycin $\mathrm{A}(25 \mu \mathrm{M})$ and rotenone $(5 \mu \mathrm{M})$.

Citrate synthase activity assay: Citrate synthase assay was performed in $100 \mathrm{mM}$ Tris- $\mathrm{HCl}, \mathrm{pH}$ 8.0, containing $1 \mathrm{mM}$ EDTA, $1 \mathrm{mM} \mathrm{5',} \mathrm{5'-dithiobis} \mathrm{2-nitrobenzoic}$ acid, $10 \mathrm{mM}$ acetyl-CoA. The reaction was initiated by addition of $10 \mathrm{mM}$ oxaloacetate. Cuvettes were warmed to $37^{\circ} \mathrm{C}$, and upon addition of $10 \mu \mathrm{g}$ of protein from WAT lysates, absorbance at $420 \mathrm{~nm}$ was measured for $10 \mathrm{~min}$. Activity is expressed as nmoles $/ \mathrm{min} / \mu \mathrm{g}$ protein.

Expression analyses: For quantitative RT-PCR, RNA was extracted from tissues using the RNeasy lipid tissue kit (Qiagen), followed by cDNA synthesis. Realtime PCR amplification was performed with SYBR Green qPCR Master Mix (SA Biosciences) using a 7900HT Fast Real-Time PCR System (Applied Biosystems) and commercially available primers for 111 , Tnfa, II6, Arg1, II10, Ym1, Hif1a, Emr1, Pgc1a, Cytc, Sirt1, Sirt3, Pdk4, Cpt1a, Cpt1b, Cox7a1, Hprt, and Idh3a (SA Biosciences). Relative expression was determined by the $2^{-\triangle \Delta C T}$ method. M1 macrophages in WAT were measured by flow cytometry as described previously (104).

For measuring protein abundance, WAT homogenates were prepared as described in Horrillo et al. (107). Equal amounts of protein were separated by SDS-PAGE, electroblotted to PVDF membranes, and probed using primary antibodies according to the manufacturers' protocol. The following antibodies were used: ALDH2 (Abcam), Sirt3 (Cell Signaling), MitoProfile ${ }^{\circledR}$ Total OXPHOS Rodent WB Antibody Cocktail (Mitosciences), COX4l1 (Cell Signaling), GAPDH 
(Cell Signaling), Parkin (Abcam), Pink1 (Cell Signaling), p62 (Cell Signaling), LC3 (Cell Signaling), protein-ubiquitin (Cell Signaling) and $\alpha$-tubulin (Cell Signaling). Fluorescent or HRP-linked secondary antibodies (Invitrogen) were used to detect and visualize the protein bands with a Typhoon 9400 variable mode imager (GE Healthcare). Band intensity was quantified using Image Quant $\mathrm{TL}^{\circledR}$ software.

Relative mitochondrial DNA (mtDNA) measurements: Mitochondrial abundance in adipose tissue was estimated by measuring mtDNA abundance, relative to nuclear DNA (nDNA) (108). Total DNA was isolated from WAT using a QIAamp DNA Mini Kit (Qiagen). A $25 \mathrm{mg}$ aliquot of the tissue was homogenized followed by overnight digestion in Proteinase $\mathrm{K}$ at $55^{\circ} \mathrm{C}$. Following isolation, relative amounts of mtDNA and $\mathrm{nDNA}$ were compared using quantitative real-time PCR using $2 \mathrm{ng}$ of the isolated DNA. Primers for cytochrome $\mathrm{b}$ (mtDNA) and $\beta$-actin (nDNA) were used; the sequences are: cytochrome b, 5'TTGGGTTGTTTGATCCTGTTTCG-3’ and 5 CTTCGCTTTCCACTTCATCTTACC-3'; $\quad$ 3 $\quad$-actin, 5 CAGGATGCCTCTCTTGCTCT-3' and 5'-CGTCTTCCCCTCCATCGT-3'.

Electron microscopy: Adipose tissues were fixed with $3 \%$ glutaraldehyde in $0.1 \mathrm{M}$ sodium phosphate buffer $(\mathrm{pH} 7.4)$ for $4 \mathrm{~h}$ at room temperature $\left(25^{\circ} \mathrm{C}\right)$. The tissues were then post-fixed with $1 \%$ osmium tetroxide for $1 \mathrm{~h}$, dehydrated, embedded in Embed-812 plastic (Electron Microscopy Sciences). Ultrathin sections were stained with uranyl acetate and Reynolds lead citrate, 
and electron micrographs were taken using a Philips CM10 transmission electron microscope operating at $80 \mathrm{kV}$.

Statistical analyses: Data are mean \pm SEM. Unpaired Student's $t$ test was used for direct comparisons. Statistical analyses for metabolomic datasets were performed using Metaboanalyst 2.0 software. A $P$ value $<0.05$ was considered significant.

\section{Results}

High fat diet increases adiposity and alters systemic metabolism: Wild-type C57BL/6J mice were placed on a LFD or HFD for 6 weeks. Significant weight gain occurred as early as 1 week on HFD, and the change in total body mass was nearly $10 \mathrm{~g}$ by 6 weeks on the diet (Figure $1 \mathrm{~A}$ ). Food and water intake were not significantly different between groups (Figure 1B\&C). Dexascan analysis showed a 2 -fold increase in \% fat mass and a concomitant decrease in \% lean mass in HF-fed mice (Figure 1D\&E). These results are typical of this commonly utilized model of diet-induced obesity $(103,109)$.

To determine how diet affects systemic metabolism, mice fed either LFD or HFD for 6 weeks were placed in metabolic chambers, and their oxygen consumption $\left(\mathrm{VO}_{2}\right)$, carbon dioxide production $\left(\mathrm{VCO}_{2}\right)$, and physical activity were measured. As shown in Figure $1 \mathrm{~F}$ and $\mathrm{G}$, average $\mathrm{VO}_{2}$ and $\mathrm{VCO}_{2}$ values decreased in HF-fed mice compared with mice fed a LFD. The respiratory exchange ratio (RER) was also decreased in HFD mice versus mice fed LFD 
(Figure $1 \mathrm{H}$ ). Physical activity, measured by total beam breaks (Figure 1I), ambulatory counts (Figure $1 \mathrm{~J}$ ), and fine movements (Figure $1 \mathrm{~K}$ ), were not significantly different between groups, although the group fed a HFD appeared to show a trend toward decreased activity. Consistent with our previous results (103), mice fed a HFD also demonstrated worsened glucose and insulin tolerance (Figure 2) as well as a significant increase in plasma insulin levels (WT LFD, $151 \pm 71 \mathrm{pg} / \mathrm{ml}$ vs. WT HFD, $2690 \pm 593 \mathrm{pg} / \mathrm{ml}, \mathrm{n}=3$ per group, $p<0.05)$. 

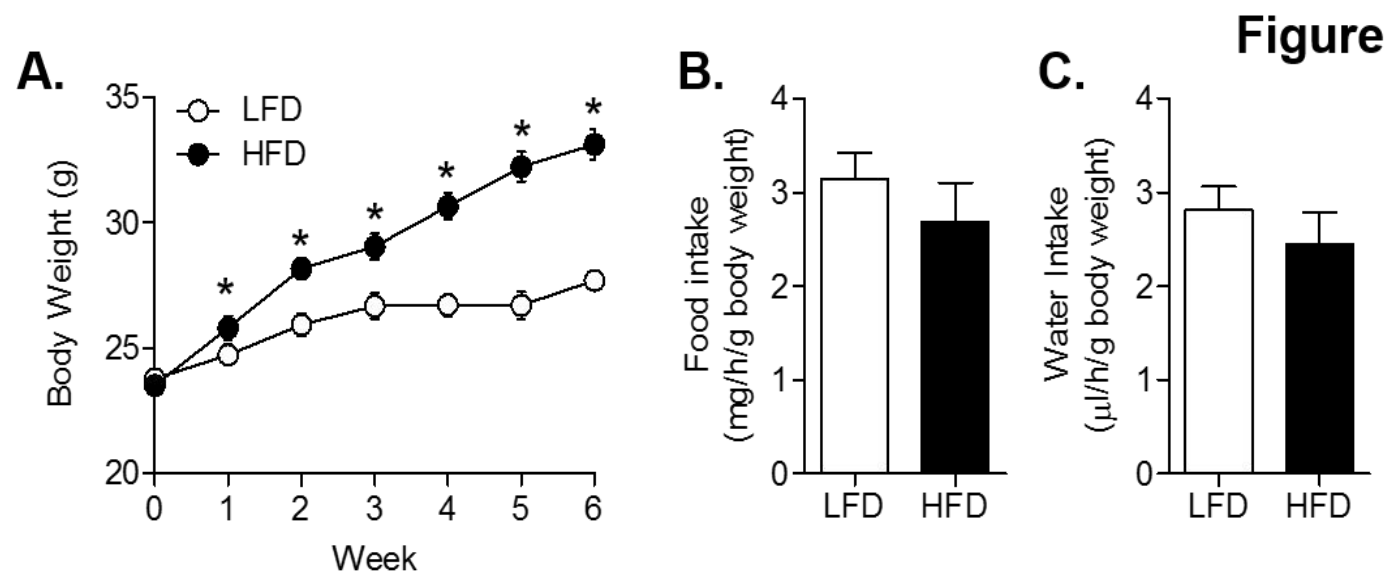

D.
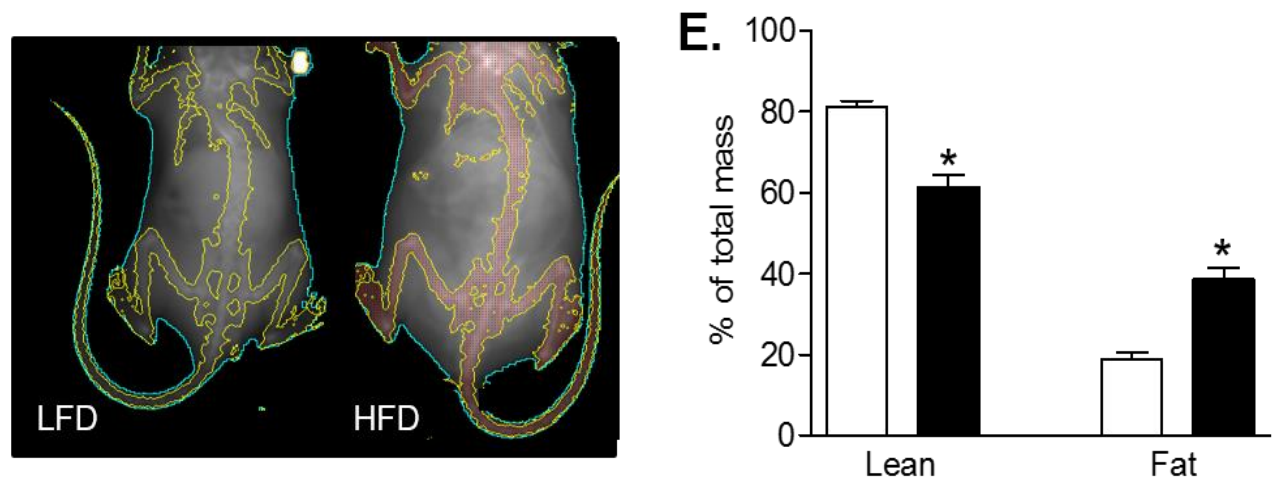

F.

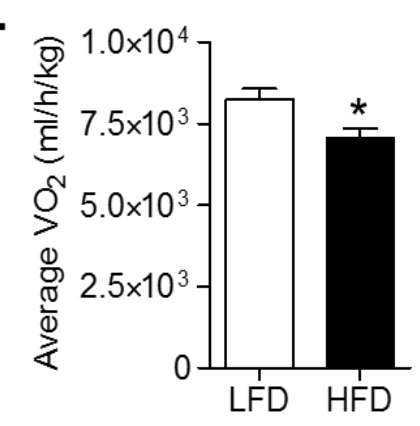

I.

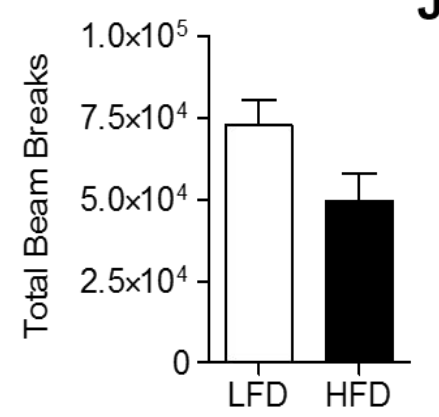

G.

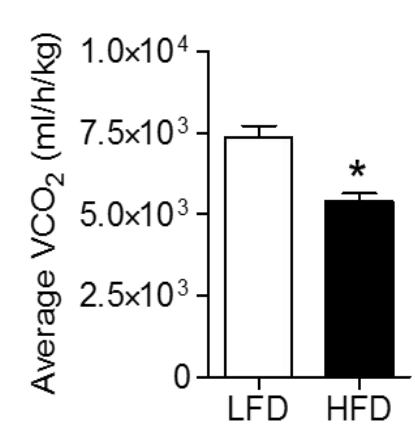

J.

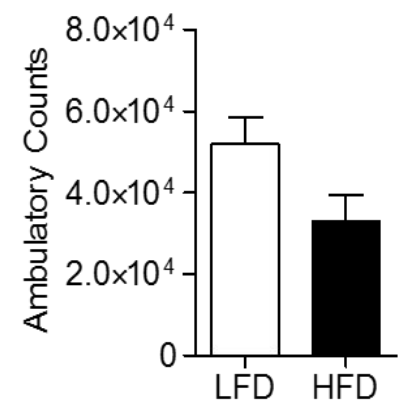

H.

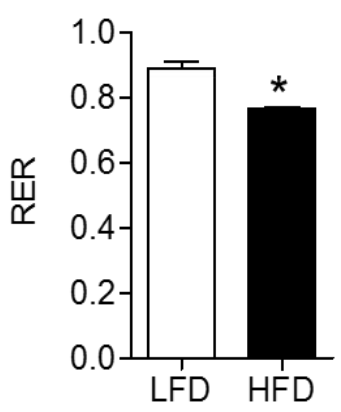

K.

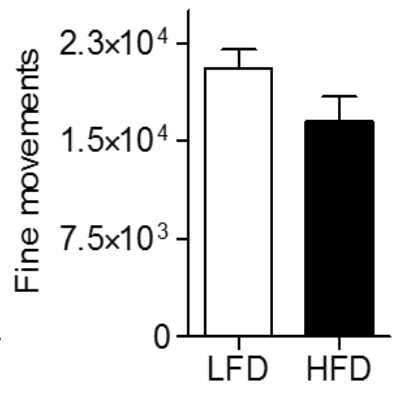


Figure 1: Effects of high fat diet on weight gain, adiposity and systemic metabolism. Male WT C57BL/6J mice were fed a low fat diet (LFD, 10\% kcal fat) or high fat diet (HFD, $60 \%$ kcal fat) for 6 weeks and the following measurements were recorded: $(\mathbf{A})$ mouse weights during 6 weeks of feeding, $n=$ 20 per group; (B) food intake, $n=7$ per group; (C) water intake, $n=7$ per group; (D) representative DexaScan images; (E) percentages of lean mass and body fat, $\mathrm{n}=10$ per group; and $(\mathbf{F})$ average oxygen consumption $\left(\mathrm{VO}_{2}\right) ;(\mathbf{G})$ average carbon dioxide production $\left(\mathrm{VCO}_{2}\right) ;(\mathrm{H})$ respiratory exchange ratio $(\mathrm{RER}) ;(\mathrm{I})$ total activity level; (J) ambulatory counts; and (K) fine movements. $\mathrm{n}=7$ per group; ${ }^{*} p<0.05$ vs. LFD. 
Figure 2
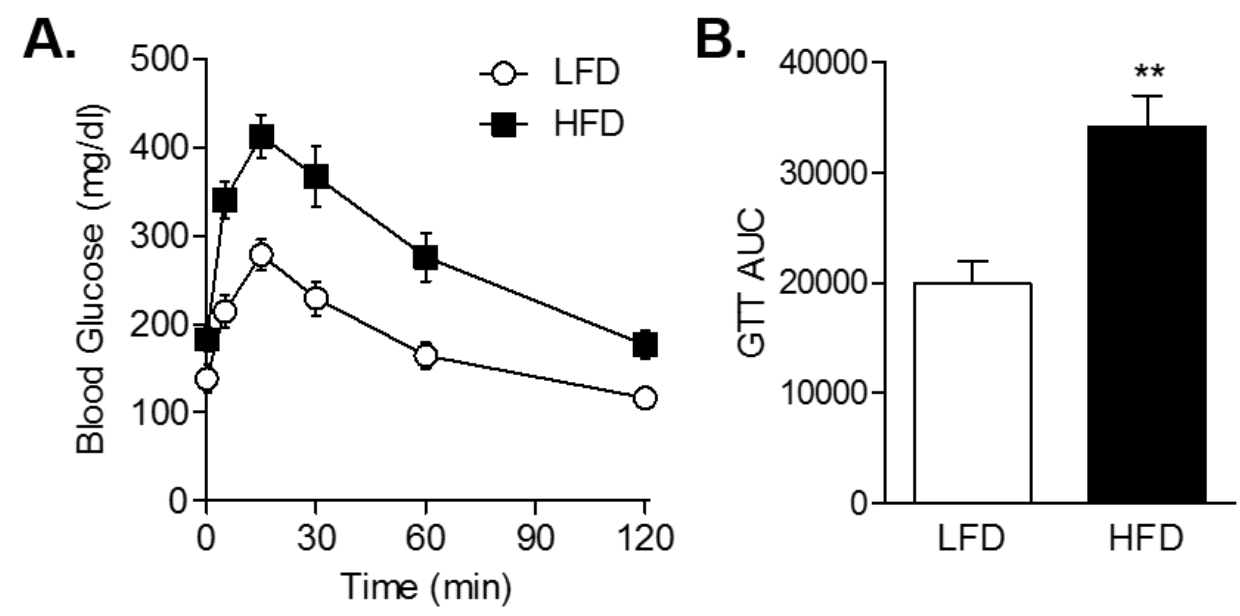

C.

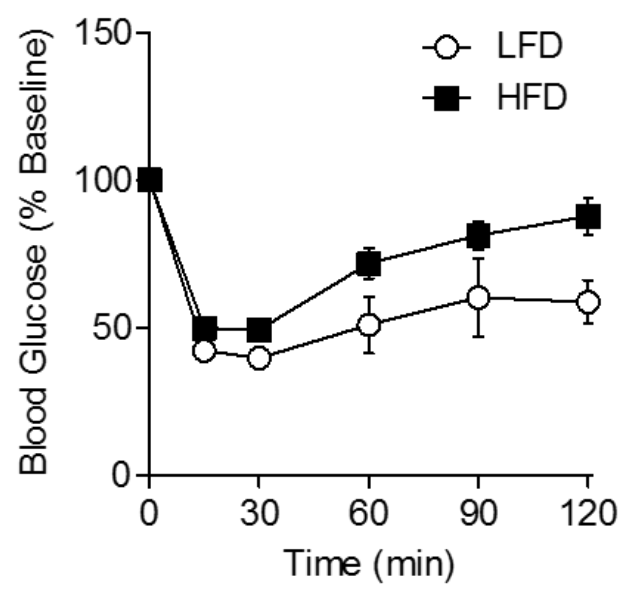

D.

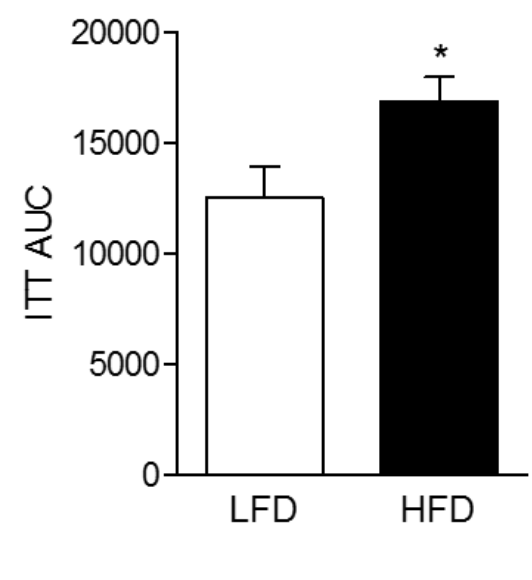


Figure 2: Glucose and insulin tolerance in mice fed low fat or high fat diets. After 6 weeks of a low fat (LFD) or high fat diet (HFD), glucose tolerance and insulin sensitivity were examined: (A) Glucose tolerance test (GTT); (B) GTT area under the curve (AUC); (C) insulin tolerance test (ITT) shown as \% of baseline; and (D) ITT AUC. $n=7$ per group; ${ }^{*} p<0.05,{ }^{* *} p<0.01$ vs. LFD. 
Robust macrophage infiltration does not occur with 6 weeks of HFD: To examine the effects of HFD on macrophage infiltration, we placed mice on LFD or HFD for 6 or 12 weeks and measured adipocyte size, crown-like structures that are indicative of macrophage infiltration, as well as the expression of inflammatory genes. As shown in Figure 3A-C, mice fed a HFD for 6 and 12 weeks showed a 3- to 4-fold increase in adipocyte size when compared with LFD controls. While sections of WAT derived from mice fed a HFD for 12 weeks showed obvious increases in crown-like structures, WAT from mice fed HFD for 6 weeks showed minimal increases in such structures. These observations suggest that with 6 weeks of HFD, there is minimal macrophage accumulation. Indeed, the expression of Emr1-a marker of macrophages-as well as that of other inflammatory genes was not changed with 6 weeks of HFD (Figure 3D). Moreover, the abundance of M1 macrophages in adipose tissue stromal vascular fractions was not different between mice fed different diets $\left(\mathrm{F} 4 / 80^{+} / \mathrm{CD} 11 \mathrm{c}^{+} / \mathrm{CD} 301^{-}\right.$cells as $\% \mathrm{~F} 4 / 80^{+}$cells: LFD $37.4 \pm 3.5 ; \mathrm{HFD}, 45.9 \pm 1.8 ; \mathrm{n}$ $=9-10$ per group, $p>0.05$.$) . Most likely, the modest, insignificant increase in$ Tnfa is due to adipocytes, which have been shown to be capable of producing TNF- $\alpha(110,111)$. In addition, no increase in plasma levels of inflammatory mediators such as IL-6 were identified at 6 weeks of HFD (IL-6 (pg/ml): LFD, 23.6 $\pm 7.5 ; \mathrm{HFD}, 18.8 \pm 4.2$ ). Collectively, these data show that adipocyte size was increased after 6 weeks of HFD, without significant changes in infiltrating inflammatory cells. 


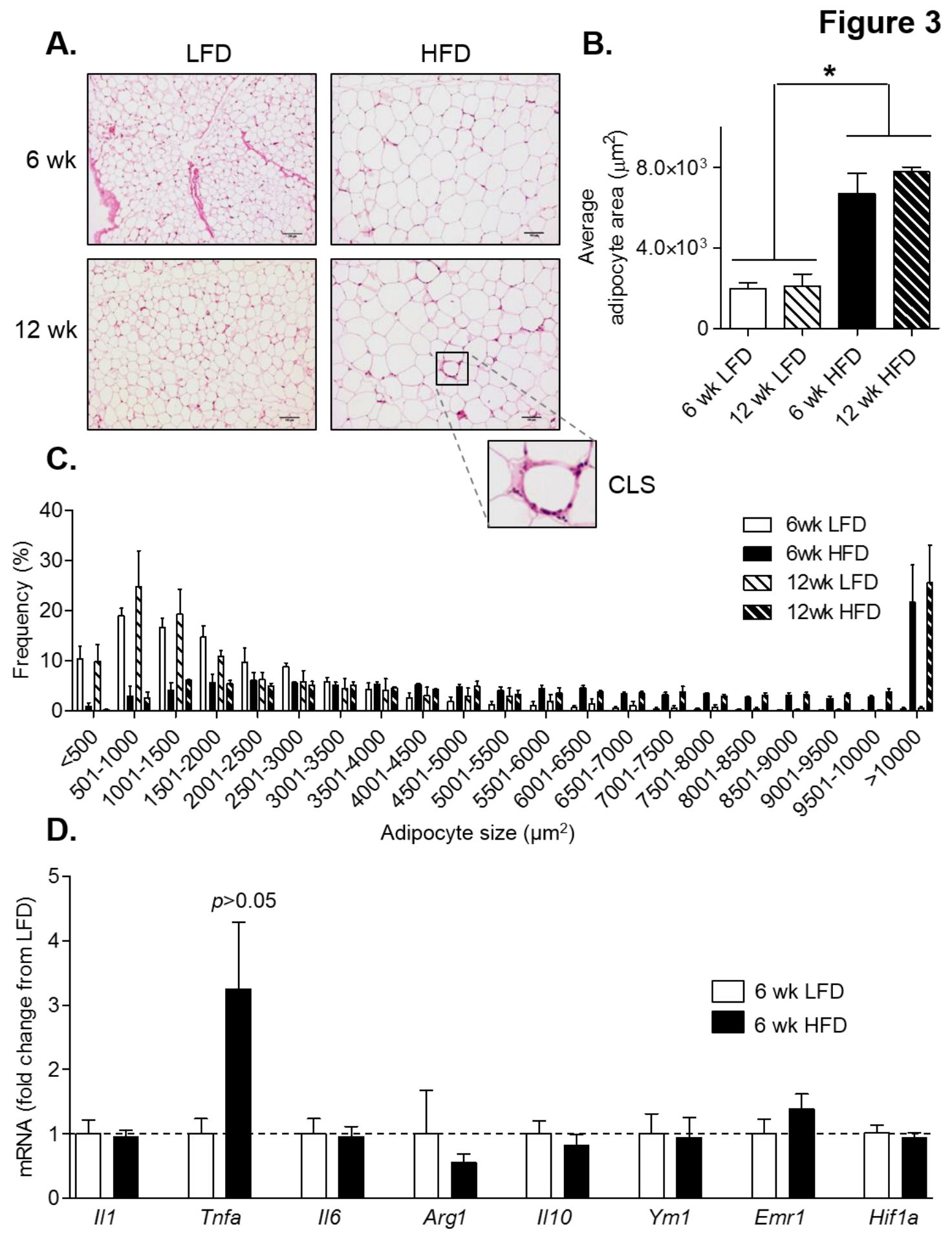


Figure 3: Effect of HFD on adipose tissue expansion and inflammation. Morphological and molecular changes in adipose tissues: (A) Representative hematoxylin and eosin stains of epididymal adipose tissue from mice fed a LFD or HFD for 6 or 12 weeks; inset shows a crown like structure (CLS) in the 12 week HFD group; (B) average area of adipocytes; (C) adipocyte size distribution; and (D) qRT-PCR analyses of markers of inflammation in adipose tissues from mice fed a LFD or HFD for 6 weeks. $n=4-5$ per group; ${ }^{*} p<0.05$ vs. indicated groups. 
Obesity alters the metabolite profile of adipose tissue: To examine the effect of obesity on adipose tissue metabolism, epididymal WAT from mice fed a LFD or HFD for 6 weeks was subjected to unbiased metabolomic analysis. The relative concentration of adipose metabolites was measured by mass spectrometry and queried against the Metabolon reference library. It is important to note that the composition of adipose tissue derived from mice fed LFD differed from mice fed HFD. In comparison with the LFD group, the protein yield per mg wet weight was $43 \%$ lower in the adipose tissue of the HFD group ( $\mu \mathrm{g}$ protein/mg wet weight: 6 wk LFD, 10.86 $\pm 1.70 ; 6$ wk HFD, 6.21 $\pm 1.28 ; n=10-12$ per group). Hence, for metabolomic analyses, we corrected for this difference in protein content. Partial least squares-discriminant analysis (PLS-DA) with the corrected data showed that the LFD samples separate clearly from HFD samples (Figure 4A), and cluster analysis showed that the abundance several metabolites was associated with diet (Figure 4B). Of the 191 metabolites measured, 49 were found to be significantly different $(p<0.05)$ in the WAT of mice fed a HFD compared with the adipose tissue from mice fed LFD. Volcano plot analysis, using data corrected for protein content, showed significantly higher levels of 34 metabolites and significantly lower levels of 15 metabolites in adipose tissue of HF-fed mice compared with those fed LFD (Figure 4C and Table 1). Volcano plot analysis based on uncorrected, raw data showed a large skew towards decreased abundance of most metabolites (Figure $4 \mathrm{C}$, inset). To visualize the data in the biological context of metabolic pathways, metabolites that were statistically different in each group were analyzed using the MetPA tool of Metaboanalyst 2.0 
software. The pathways were calculated as the sum of the importance measures of the matched metabolites normalized by the sum of the importance measures of all metabolites in each pathway $(105,112)$. As shown in Figure 4D, the highest pathway impact values were related to linoleic acid metabolism and Phe, Tyr, and Trp metabolism. Branched chain amino acids (BCAA) i.e., Val, Leu, and Ile, and pathways related with taurine metabolism; glycerolipid metabolism; Ala, Asp and Glu metabolism; carbohydrate metabolism; and pyrimidine metabolism also showed relatively high pathway impact and significance values. 
Figure 4

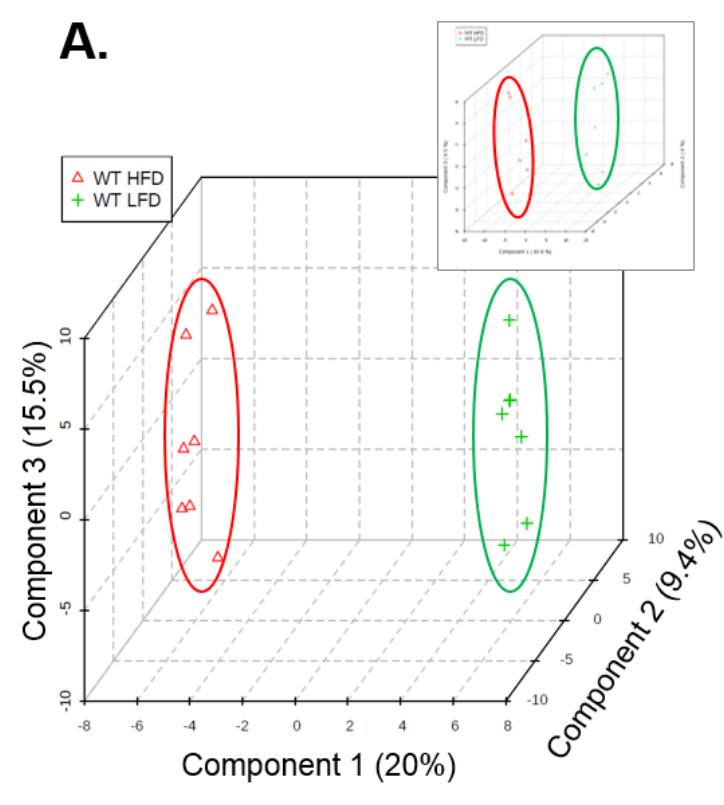

c.

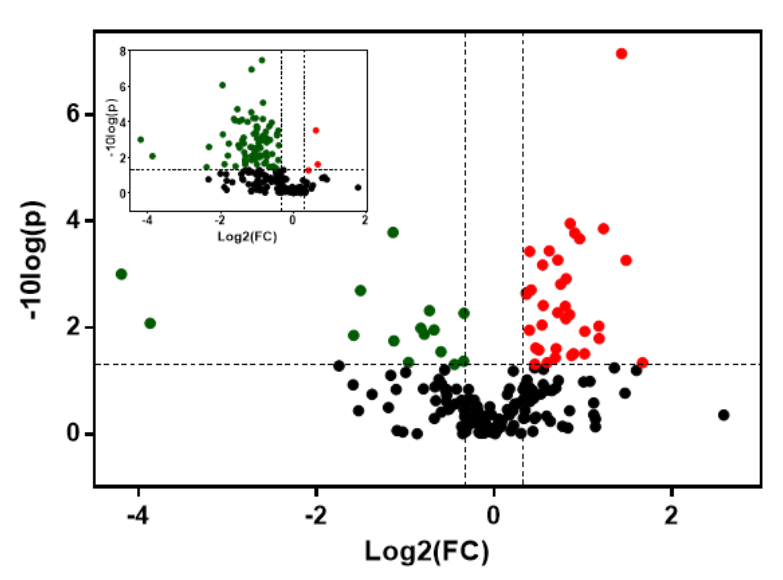

B.

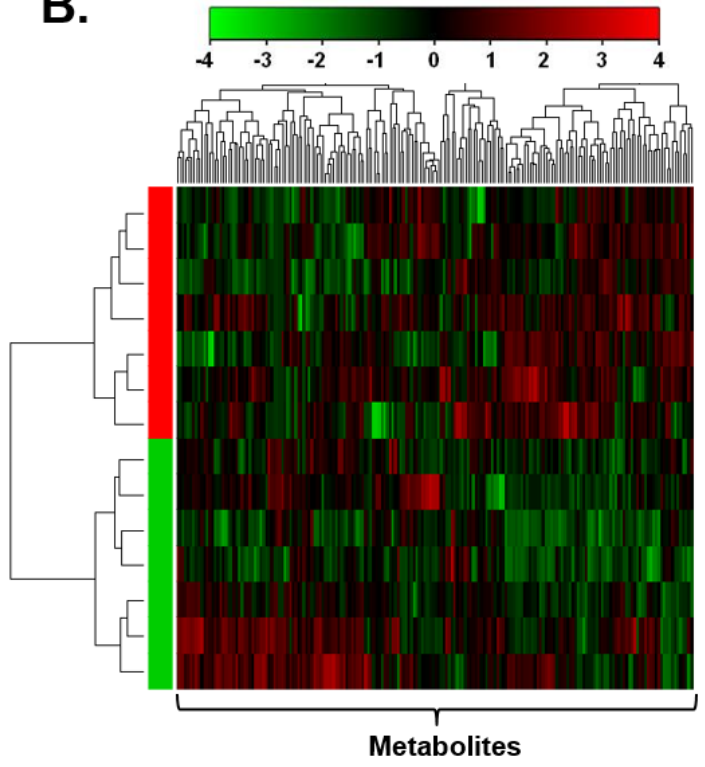

D.

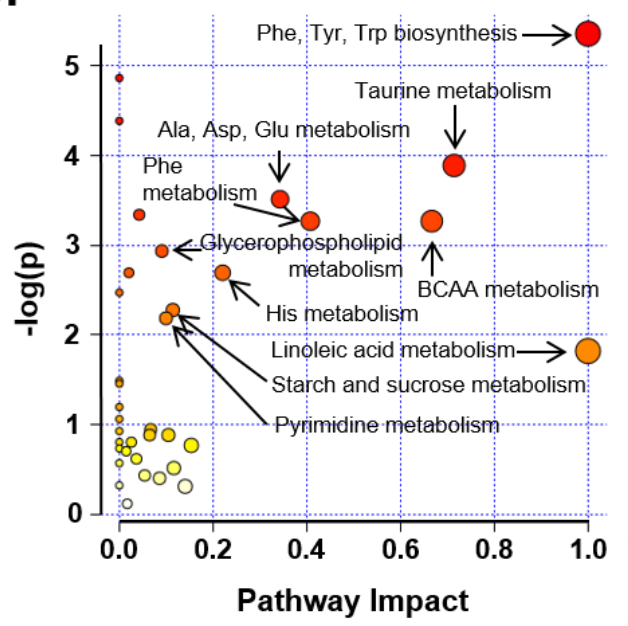


Figure 4: Metabolomic analyses of adipose tissue. Metabolomic analyses of epididymal adipose tissue metabolites from WT mice fed a LFD or HFD for 6 weeks: (A) Multivariate analysis: partial least squares-discriminant analysis (PLSDA); inset shows PLS-DA analysis from the uncorrected dataset (B) Hierarchial clustering: Heatmap and dendogram; (C) Univariate analysis: Volcano plot of metabolites. Those metabolites that were significantly higher in abundance are in the quadrant shaded red and those that were significantly lower are shaded green $(p<0.05$, t-test). The identity of these metabolites is found in Table 1 . The inset shows volcano plot analysis using data that were not corrected for protein content; (D) Metabolites found to be significantly different were subjected to pathway impact analysis using Metaboanalyst MetPA and the Mus musculus pathway library. Fisher's exact test was used for overrepresentation analysis, and relative betweenness centrality was used for pathway topology analysis. $n=$ 14 animals: 7 WT LFD and 7 WT HFD. 
Table 1

\begin{tabular}{|c|c|c|c|}
\hline Metabolite & $\begin{array}{c}\text { Fold } \\
\text { change }\end{array}$ & $p$ value & FDR \\
\hline *stearoyl sphingomyelin & 2.8 & 0.000542 & 0.010414 \\
\hline *glycerophosphoethanolamine & 2.7 & 7.09E-08 & 1.36E-05 \\
\hline *succinate & 2.34 & 0.000139 & 0.006385 \\
\hline inosine 5'-monophosphate (IMP) & 2.27 & 0.016131 & 0.088492 \\
\hline uridine monophosphate ( $5^{\prime}$ or $\left.3^{\prime}\right)$ & 2.26 & 0.009442 & 0.064744 \\
\hline dihomo-linoleate $(20: 2 n 6)$ & 2.03 & 0.011868 & 0.071209 \\
\hline pelargonate $(9: 0)$ & 2.02 & 0.030984 & 0.14203 \\
\hline *cytidine 5'-monophosphate (5'-CMP) & 1.95 & 0.000214 & 0.006839 \\
\hline $\begin{array}{l}\text { *Isobar: fructose } 1,6 \text {-diphosphate, glucose } 1,6 \text { - } \\
\text { diphosphate, myo-inositol } 1,4 \text { or } 1,3 \text {-diphosphate }\end{array}$ & 1.87 & 0.000166 & 0.006385 \\
\hline maltose & 1.86 & 0.03107 & 0.14203 \\
\hline docosapentaenoate (n3 DPA; 22:5n3) & 1.83 & 0.033432 & 0.14928 \\
\hline *glutamate & 1.81 & 0.000111 & 0.006385 \\
\hline glucose & 1.8 & 0.005764 & 0.046111 \\
\hline azelate (nonanedioate) & 1.75 & 0.001215 & 0.01795 \\
\hline dihomo-linolenate $(20: 3 n 3$ or n6) & 1.75 & 0.006864 & 0.052713 \\
\hline stearate $(18: 0)$ & 1.74 & 0.003972 & 0.038131 \\
\hline arachidonate $(20: 4 n 6)$ & 1.68 & 0.001536 & 0.021059 \\
\hline deoxycarnitine & 1.64 & 0.000539 & 0.010414 \\
\hline glycerophosphorylcholine (GPC) & 1.64 & 0.005243 & 0.045087 \\
\hline hypotaurine & 1.62 & 0.024921 & 0.12592 \\
\hline 12-HETE & 1.61 & 0.036945 & 0.16121 \\
\hline acetylcarnitine & 1.54 & 0.000362 & 0.008923 \\
\hline margarate $(17: 0)$ & 1.51 & 0.046138 & 0.18455 \\
\hline aspartate & 1.47 & 0.003862 & 0.038131 \\
\hline creatine & 1.46 & 0.000666 & 0.01162 \\
\hline malate & 1.45 & 0.008981 & 0.063867 \\
\hline linoleate $(18: 2 n 6)$ & 1.42 & 0.026561 & 0.13076 \\
\hline palmitoyl ethanolamide & 1.38 & 0.02444 & 0.12592 \\
\hline
\end{tabular}




\begin{tabular}{l|rrc}
\hline propionylcarnitine & 1.37 & 0.048868 & 0.18902 \\
taurine & 1.33 & 0.001968 & 0.024159 \\
glutamine & 1.32 & 0.000372 & 0.008923 \\
choline phosphate & 1.32 & 0.011199 & 0.06936 \\
citrulline & 1.29 & 0.002367 & 0.025244 \\
cholesterol & 1.28 & 0.002251 & 0.025244 \\
*leucine & 0.79 & 0.005401 & 0.045087 \\
*tyrosine & 0.78 & 0.042828 & 0.18273 \\
pantothenate & 0.73 & 0.049223 & 0.18902 \\
mead acid (20:3n9) & 0.66 & 0.028737 & 0.13794 \\
phenylalanine & 0.62 & 0.011086 & 0.06936 \\
valine & 0.6 & 0.00483 & 0.044159 \\
3-dehydrocarnitine & 0.58 & 0.013332 & 0.077568 \\
1,5-anhydroglucitol (1,5-AG) & 0.56 & 0.010344 & 0.068482 \\
glucose-6-phosphate (G6P) & 0.51 & 0.045306 & 0.18455 \\
histamine & 0.46 & 0.017878 & 0.095349 \\
ergothioneine & 0.45 & 0.000163 & 0.006385 \\
*N-acetylmethionine & 0.35 & 0.002013 & 0.024159 \\
*mannose-6-phosphate & 0.33 & 0.013998 & 0.079048 \\
*glycerate & 0.07 & 0.008323 & 0.061462 \\
\hline & 0.000986 & 0.015774 \\
\hline
\end{tabular}


Table 1. List of adipose tissue metabolites that changed significantly in high fat-fed mice. Wild-type (WT) mice were fed a low fat or high fat diet (LFD or HFD, respectively) for 6 weeks. Epididymal adipose tissue was then subjected to LC or GC mass spectrometric analysis. Raw spectral values from WAT of HFfed mice were corrected based on the mean difference in protein content (per mg wet weight) compared with WAT from LF-fed mice. Adipose tissue metabolites in HF-fed mice that were at least $25 \%$ changed in abundance and significantly different $(p<0.05)$ compared with LF-fed mice are shown. $n=7$ mice per group; *indicates those metabolites that were significant even when \pm 2 SEM were applied in protein correction. 
To delineate changes in metabolites further, metabolites were categorized based on superfamily class, and z-score plots were constructed. As shown in Figure 5, the abundance of multiple lipid superfamily members, including those involved in glycerolipid metabolism and linoleic acid metabolism was higher. Several carnitine derivatives, with the sole exception of 3-dehydrocarnitine, were higher in abundance as well. In the amino acid superfamily, the levels of non-essential amino acids Glu, Gln and Asp were elevated, and those of essential amino acids Phe, Leu and Val were lower. Notably, the organic osmolytes-taurine, hypotaurine and creatine-were more abundant. In the HFD group, levels of the Krebs cycle intermediate succinate were higher; levels of malate were higher as well, although pantothenate, a precursor to Coenzyme A (CoA), was less abundant. While both glucose and maltose levels were higher, phosphorylated sugars were diminished. Plasma 1,5-anhydroglucitol (1,5-AG), which is present in most organs and tissues and has been shown to decrease with loss of glycemic control $(113,114)$, was diminished in abundance, which is in agreement with insulin resistance in skeletal muscle and liver occurring at 6 weeks of HFD (109). Interestingly, the pyrimidine monophosphates cytidine-5'-monophosphate (CMP) and uridine monophosphate (UMP), as well as the purine inosine-5'monophosphate, were elevated in WAT from HF-fed mice. A graphical illustration of these changes, placed into context of known pathways of intermediary metabolism, is shown in Figure 6. 


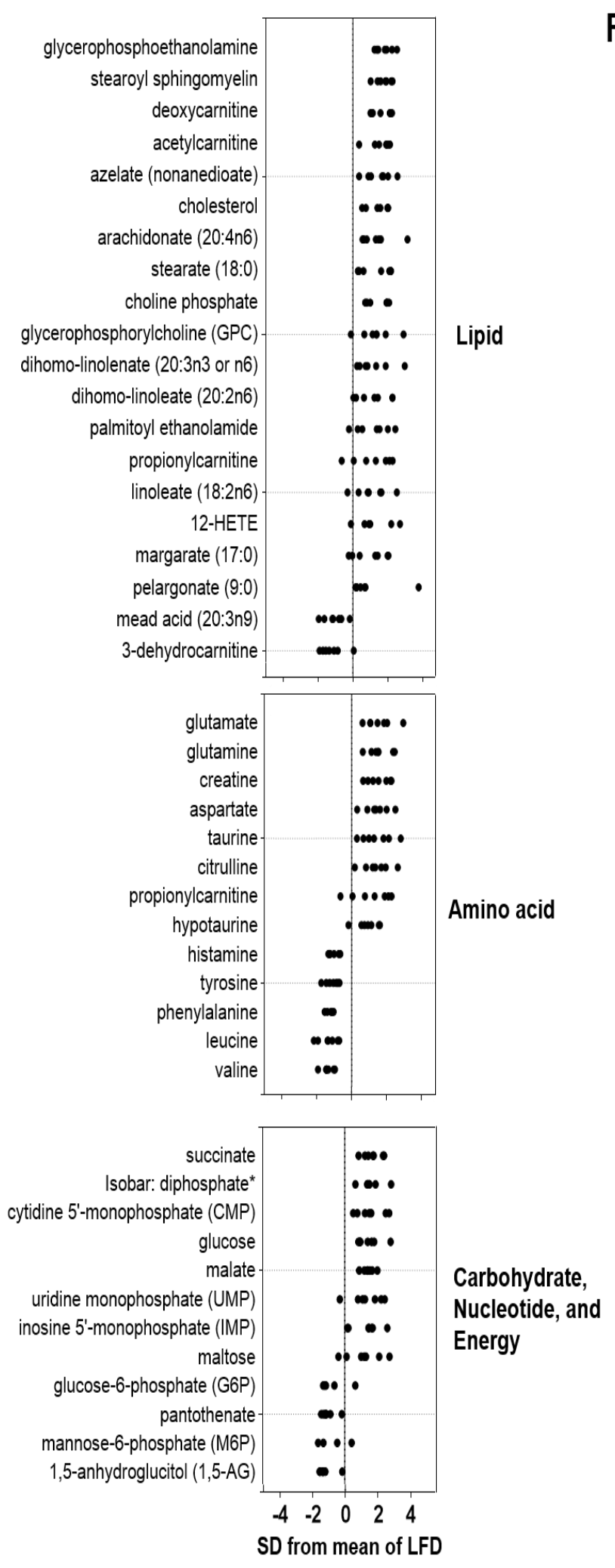


Figure 5: Z-score plot analysis of metabolite changes in adipose tissue from high fat fed mice. Mice were fed a LFD or HFD for 6 weeks. Data are shown as standard deviations from the mean of LFD. Z-scores of some metabolites were not plotted due to lack of detectable signal in either LFD or HFD groups, e.g., glycylleucine, glycerate. Each point represents one metabolite in one sample. $\mathrm{n}=7$ per group. 
Figure 6

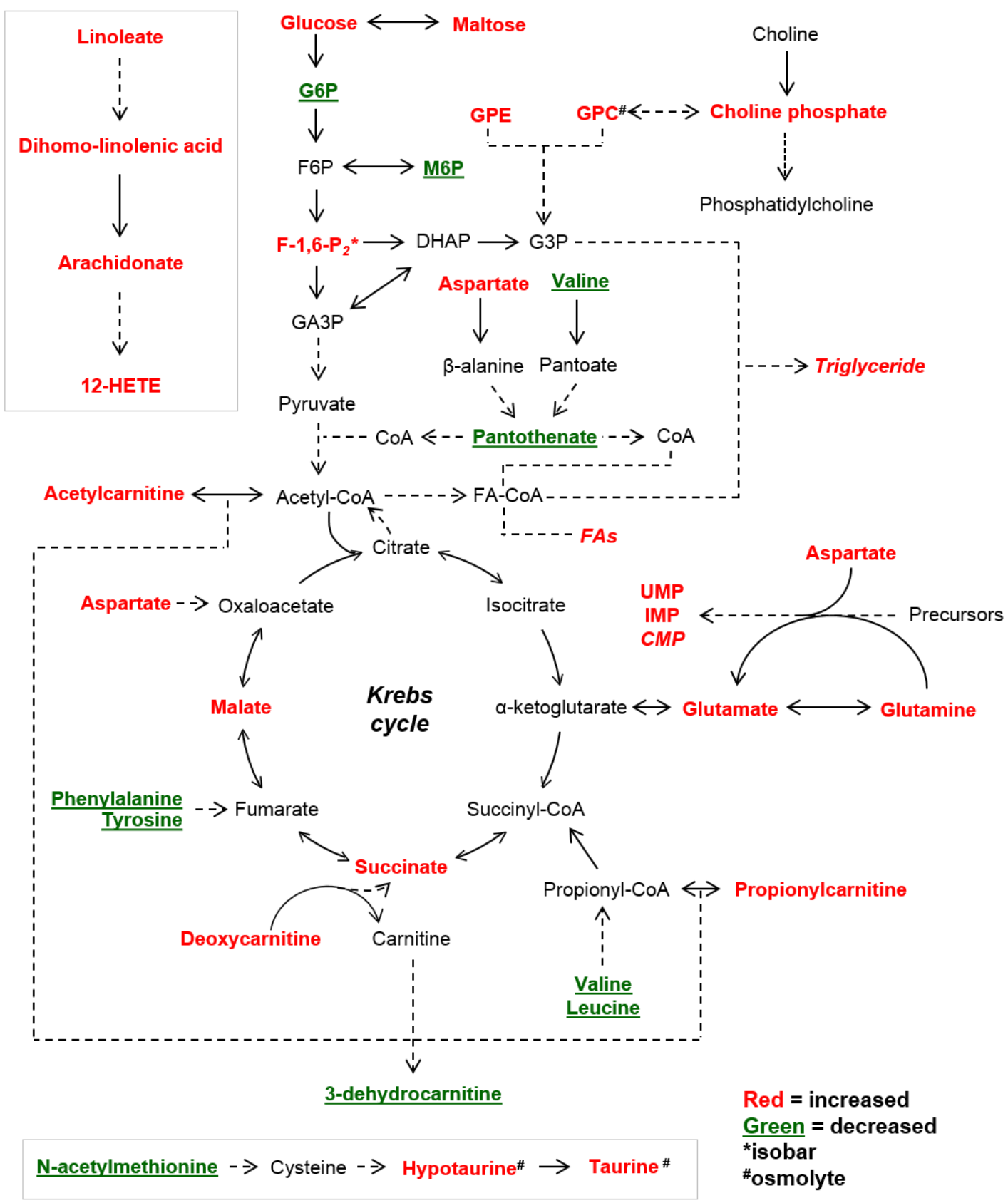


Figure 6: Illustration of metabolite changes in adipose tissues of obese mice. Metabolic changes in adipose tissue that occur with HFD are shown in the context of known metabolic pathways. In red are those metabolites that increased, and in green (and underlined) are those metabolites that decreased, in obese mice. 12-HETE, 12-Hydroxyeicosatetraenoic acid; BCAAs, branched chain amino acids; CoA, coenzyme A; CMP, cytidine 5'-monophosphate; DHAP, dihydroxyacetone phosphate; FAs, fatty acids; F6P, fructose-6-phosphate; F1,6- $\mathrm{P}_{2}$, fructose-1,6-bisphosphate; G3P, glycerol-3-phosphate; G6P, glucose-6phosphate; GA3P, glyceraldehyde-3-phosphate; GPC, glyerophosphorylcholine; GPE, glycerophosphoethanolamine; IMP, inosine 5'-monophosphate; UMP, uridine monophosphate (5' or $\left.3^{\prime}\right)$. *isobaric entity: Metabolite identity is unclear from the data and could be either F-1,6- $\mathrm{P}_{2}$, glucose-1,6-diphosphate, or myoinositol 1,4 or 1,3 diphosphate; "denotes organic osmolytes that changed in abundance. 
Effect of obesity on oxygen consumption and mitochondrial remodeling in WAT: The changes in energy metabolism found in our metabolomic analyses suggested that HFD might have altered adipose tissue bioenergetics. Importantly, these changes occurred in the absence of inflammatory cell infiltration (see Figure 3), which could otherwise confound adipocyte-specific changes in metabolism. To determine how obesity affects mitochondrial function, WAT explants from mice fed a LFD or HFD were subjected to extracellular flux analysis. As shown in Figure 7A and B, the apparent basal mitochondrial oxygen consumption rate of adipose tissue derived from mice fed a LFD was $>2$-fold higher when compared with adipose explants derived from HF-fed mice $(p<0.05)$; however, after correction for protein content, there was no difference in mitochondrial oxygen consumption. Nevertheless, explants derived from mice fed a HFD responded more strongly to FCCP (Figure 7C). Although citrate synthase activity was lower by more than $50 \%$ in WAT derived from these mice (Figure 7D), relative abundance of mtDNA, as assessed by qPCR of mtDNA normalized to nDNA, was not affected with 6 weeks of HFD (Figure 7E). 
Figure 7
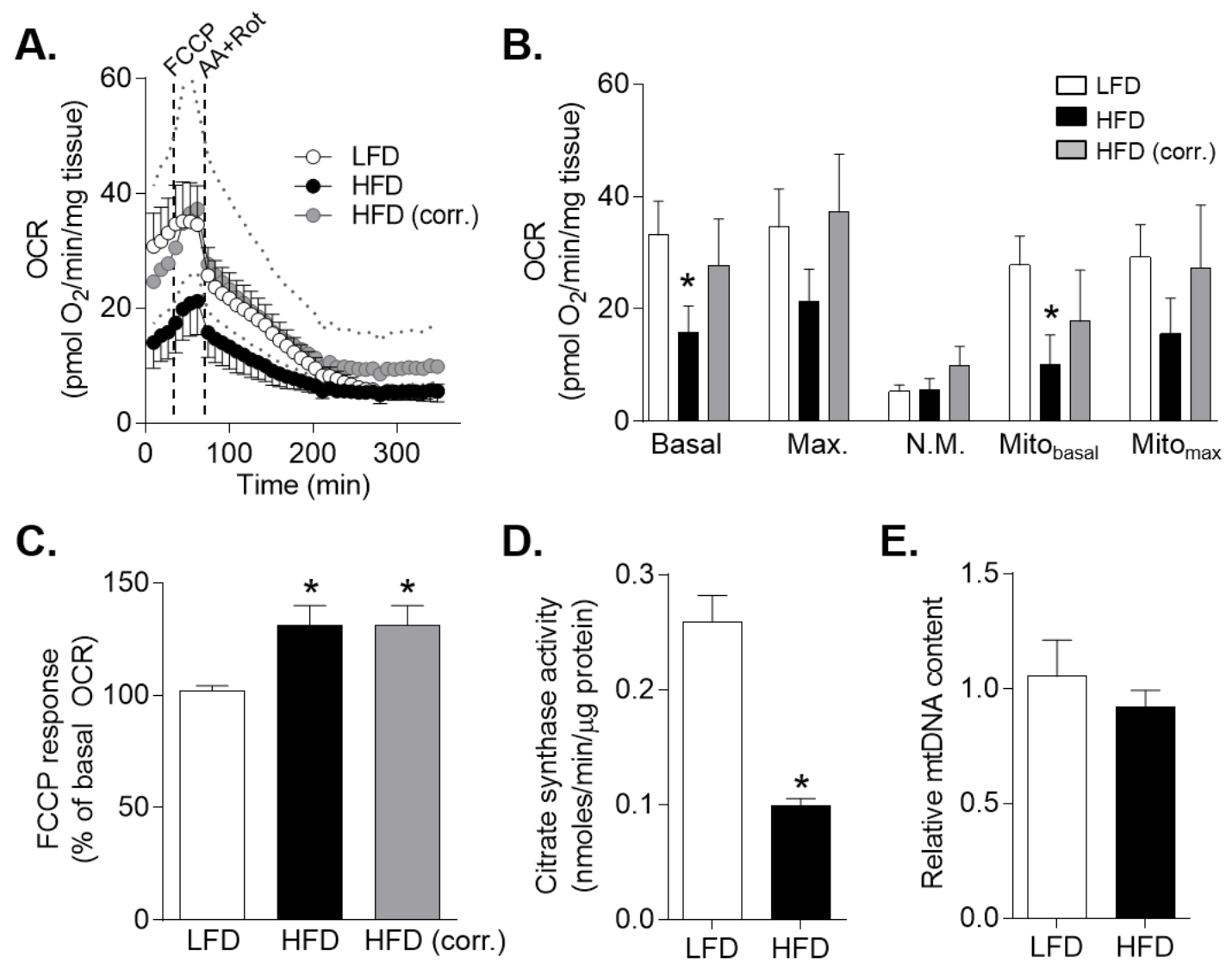
Figure 7: Obesity-related energetic changes in white adipose tissue.

Metabolic analysis of adipose tissue from mice fed a LFD or HFD for 6 weeks:

(A) Extracellular flux analysis: After three basal oxygen consumption rate (OCR) measurements, FCCP $(10 \mu \mathrm{M})$ was injected, followed by injection of antimycin A (AA, $25 \mu \mathrm{M})$ and rotenone (Rot, $5 \mu \mathrm{M})$. Gray circles indicate values from HF-fed mice that were corrected based on differences in protein content compared with LF-fed mice. For clarity, error bars were omitted from the HFD corrected group. Dotted lines indicate $\pm 2 S E M$ from corrected values. (B) Detailed changes in basal, maximal (Max.), non-mitochondrial (N.M.), basal mitochondrial (Mito basal), and maximum mitochondrial (Mito $\max$ ) rates of oxygen consumption; (C) FCCP response: the FCCP response in each explant was calculated using the equation: $\left(\mathrm{OCR}_{\mathrm{MAX}} / \mathrm{OCR} \mathrm{R}_{\mathrm{BASAL}}\right) \times 100 ; \mathrm{n}=10$ mice per group; $(\mathrm{D})$ citrate synthase activity, $n=3-6$ mice per group; (E) Relative mtDNA content, $n=6$ per group. ${ }^{*} p<0.05$ vs. LFD group. 
The observed changes in metabolite profile, increased mitochondrial responsiveness to FCCP, and diminished citrate synthase activity suggested that HFD might affect the expression of genes associated with metabolic activity. Indeed, the expression of Cox7a1, a subunit in the electron transport chain, was more than 2-fold higher in adipose tissue from mice fed a HFD, whereas the expression of Pgc1a, Sirt3, and Pdk4 expression was diminished (Figure 8A), indicating mitochondrial remodeling despite preserved basal mitochondrial activity.

To further understand how WAT mitochondria change with obesity, we assessed the relative abundance of several mitochondrial complex proteins as well as mitochondrial matrix proteins. Although no changes in mitochondrial protein abundance were observed at 6 weeks of HFD, the protein levels of NDUFB8, SDHB, and COX4I1-subunits of complexes I, II, and IV, respectively—were diminished significantly by 12 weeks of HFD (Figure 8B-E). The levels of the matrix proteins - ALDH2 and Sirt3 - showed similar trends, with ALDH2 being significantly lower after 12 weeks of HFD. 
Figure 8

A.
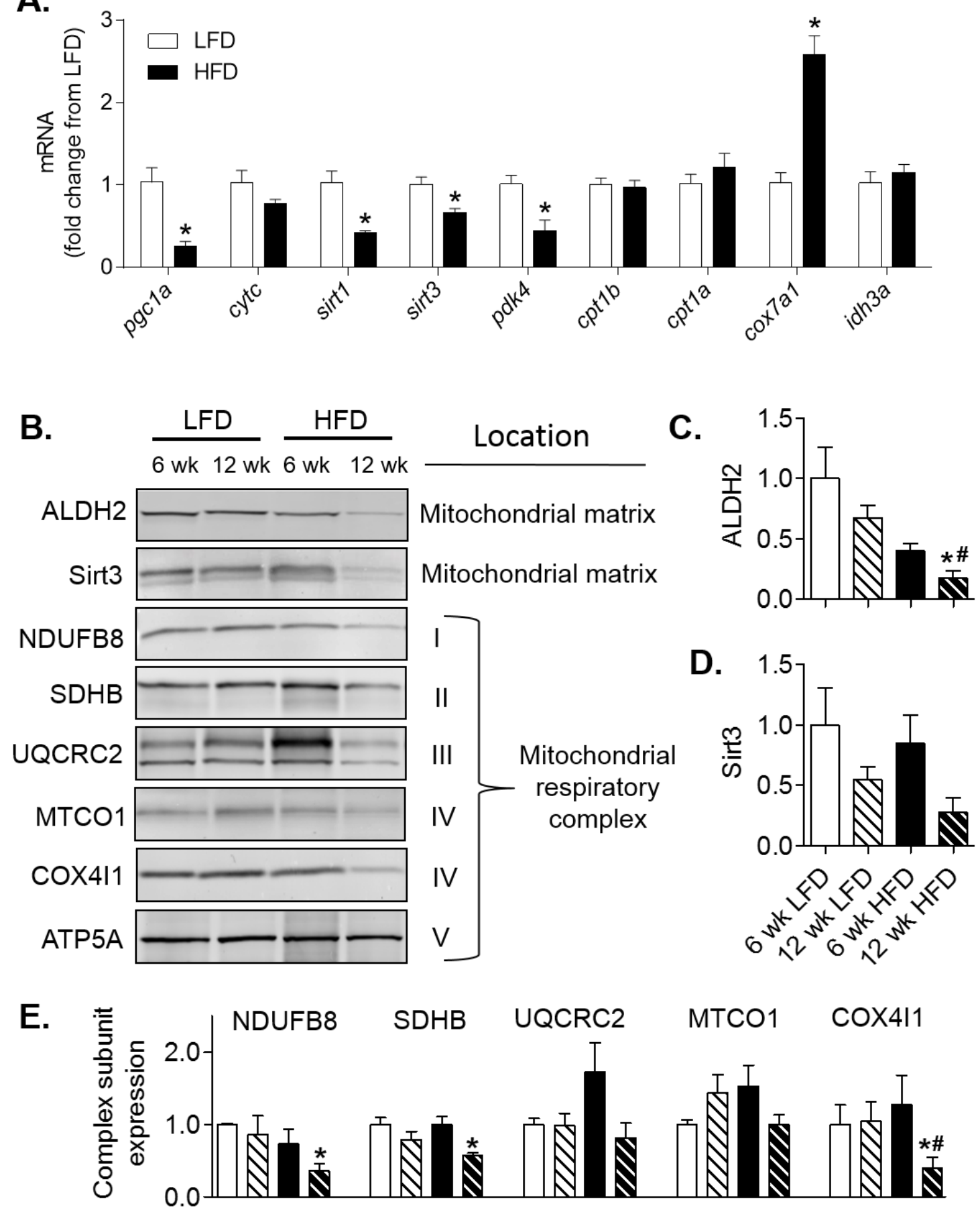
Figure 8: Obesity-related changes in mitochondrial protein abundance in white adipose tissue. Analysis of gene expression and protein abundance adipose tissue from mice fed a LFD or HFD for 6 or 12 weeks: (A) Expression of metabolic genes; (B) Representative Western blots of mitochondrial matrix proteins and respiratory chain subunits; (C) Quantification of ALDH2; (D) Quantification of Sirt3; (E) Quantification of respiratory subunit abundance. All blots were normalized to ATP5A, which showed no change in abundance in any group. $\mathrm{n}=4$ per group; ${ }^{*} \mathrm{p}<0.05$ vs. 6 wk LFD, ${ }^{*} \mathrm{p}<0.05$ vs 12 wk LFD. 
Assessment of adipose tissue ultrastructure: To determine subcellular changes that occur in adipose tissue of nutrient-stressed mice, we examined adipocyte ultrastructure using electron microscopy. As shown in Fig 9A, adipose tissue from mice fed a LFD showed mitochondria with three distinct morphologies: a round morphology of small size that was located near the nucleus (Figure 9A-i,ii); a typical elongated shape up to $\sim 0.7 \mu \mathrm{m}$ in length located in small protrusions along the adipocyte cell membrane (Figure 9A-iii); and long mitochondria (up to 5 $\mu \mathrm{m}$ and above), which were located in juxtaposition to the fat locule (Figure 9Aiv). In adipocytes derived from HF-fed mice, autophagosomes-defined by a double-membrane and comprising cytoplasmic constituents-were found next to mitochondria (Figure 9B-i), and large vacuoles of electron-dense material were found adjacent to autophagosomes (Figure 9B-ii,iii). In addition, many mitochondria in adipose tissues from HF-fed mice appeared to be undergoing fission (Figure 9B-iv,v). 
A.

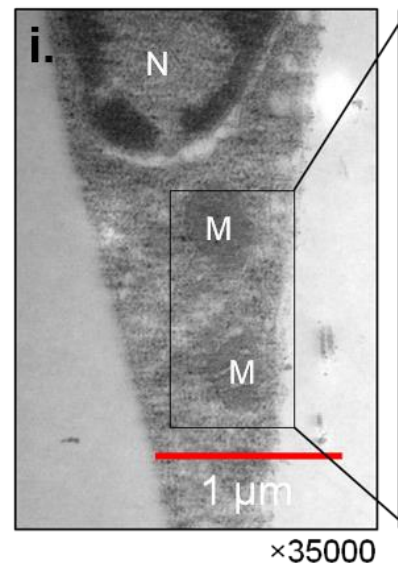

B.
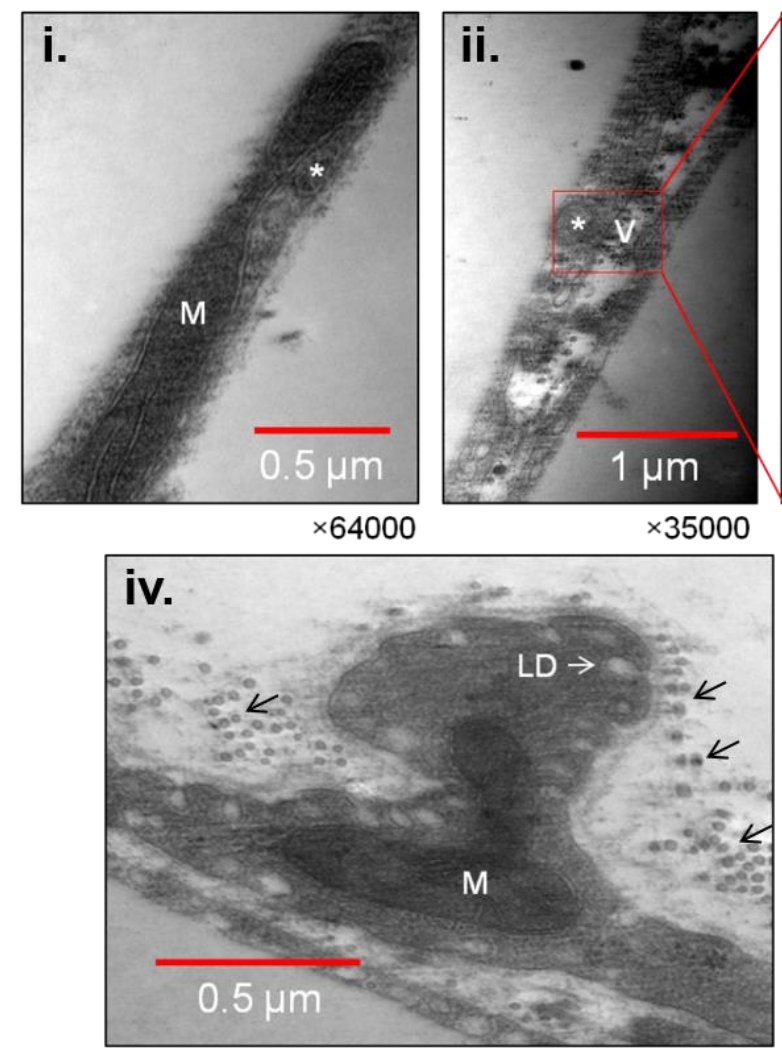

$\times 27500$

HFD

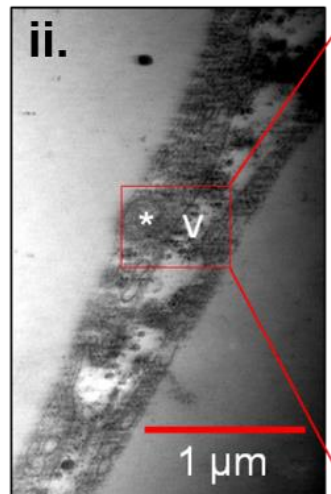

Figure 9

LFD
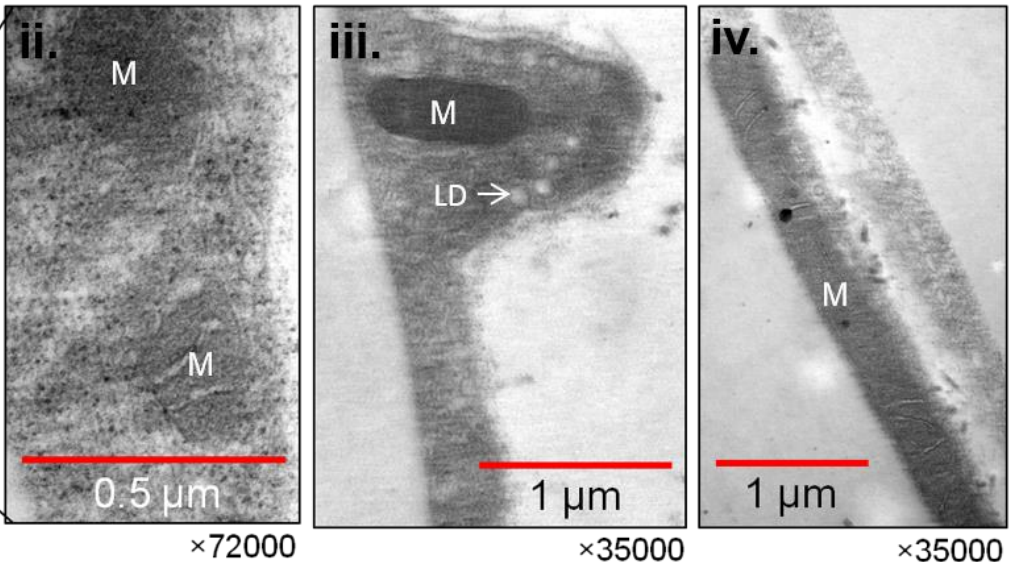
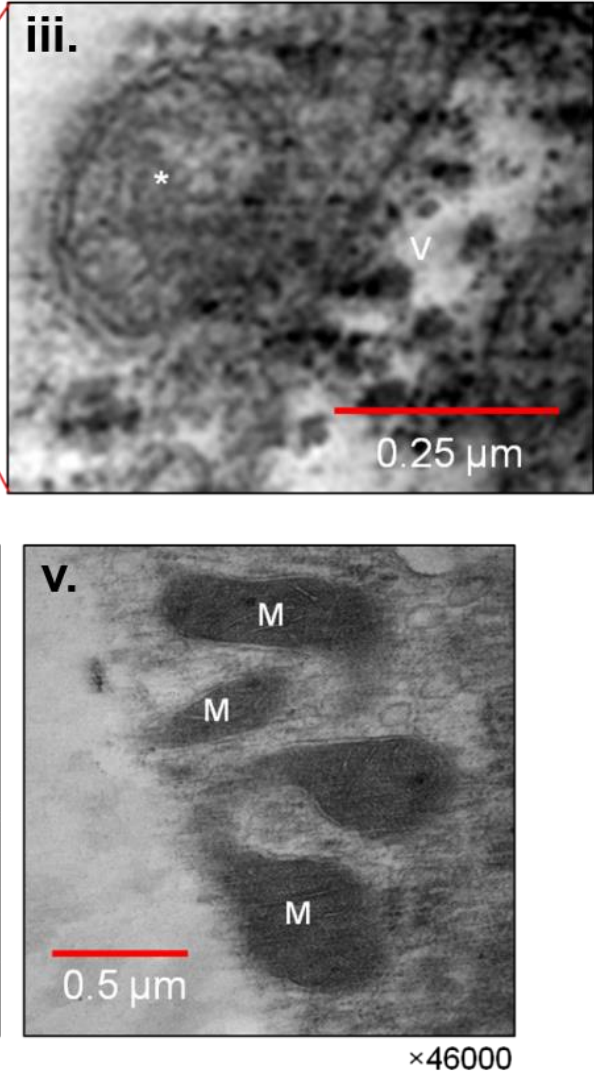
Figure 9: Ultrastructure of white adipose tissues from lean and obese mice. Representative transmission electron micrographs of epididymal adipose tissues derived from mice fed a LFD or HFD for 6 weeks. (A) Ultrastructure of mitochondria in adipose tissues from LF-fed mice: (i) micrograph of adipocytes in areas close to the nucleus; (ii) higher magnification of panel i; (iii) a cytosolic compartment containing a mitochondrion found protruding into the fat locule; and (iv) an elongated mitochondrion in juxtaposition to the fat locule. (B) Ultrastructure of adipose tissue derived from HF-fed mice: (i) An elongated mitochondrion next to an autophagosome; (ii) an autophagosome in close proximity to a vacuole containing electron-dense material; (iii) magnified image of panel ii; (iv) protrusion of cytosolic compartment containing an atypical mitochondrion; and (v) mitochondrion that appears to be undergoing fission. Asterisks $\left(^{*}\right)$ indicate autophagosomes; small arrows indicate collagen; mitochondria (M), nucleus (N), vacuole lipid droplet (LD). 
Effects of HFD on autophagy: Changes in citrate synthase and mitochondrial proteins, along with the ultrastructural alterations found to occur in the adipose tissue, suggest that HFD may promote mitochondrial remodeling and activate mitophagy in WAT. To examine this possibility, we measured markers of mitophagy and autophagy in adipose tissues from mice fed a LFD or HFD for 6 weeks. Levels of the E3 ubiquitin ligase Parkin, which has been shown to accumulate in mitochondria destined for degradation (115), were 2.3-fold higher with 6 weeks of HFD and nearly 2-fold higher after 12 weeks of HFD (Figure 10A,B). Furthermore, the kinase Pink1—critical for identifying mitochondria destined for autophagy (115)—was also nearly $40 \%$ higher in the HFD groups. In combination with the presence of autophagosomes and mitochondrial alterations observed by EM (Figure 9), this observation suggests that metabolic remodeling of adipocytes in the expanding adipose organ may be related to autophagy. To address this possibility, we measured changes in protein indicators of autophagy. As shown in Figure $10 \mathrm{C}-\mathrm{H}$, levels of p62 and LC3-I were diminished significantly and the LC3-II:LC3-I ratio was more than 2-fold higher in mice fed a HFD for 6 weeks in comparison with those placed on LFD. However, there was no significant difference in total protein abundance of protein-ubiquitin and LC3-II. 
Figure 10

A.

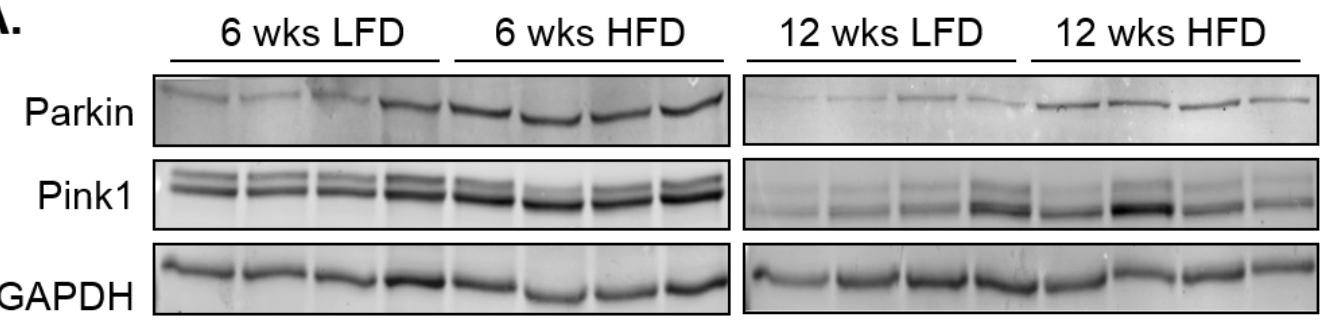

B.
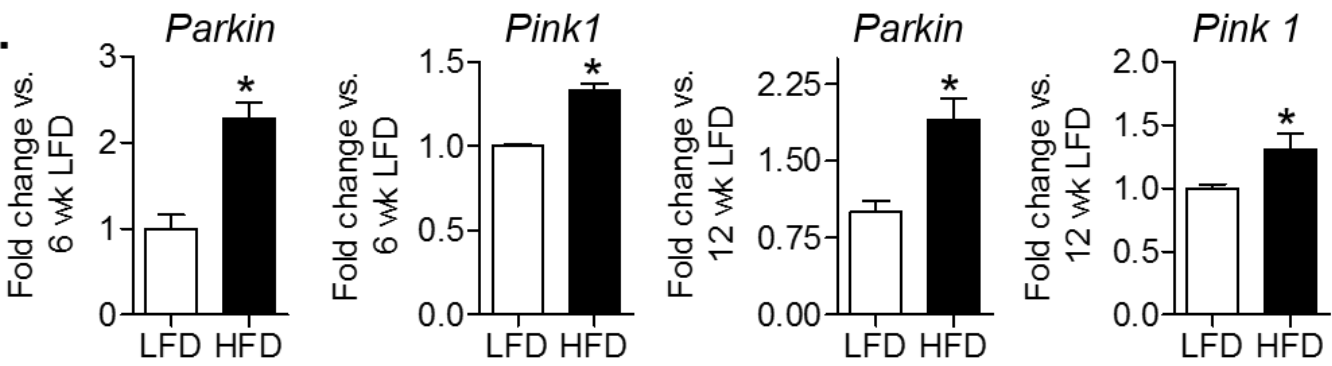

c.

Proteinubiquitin
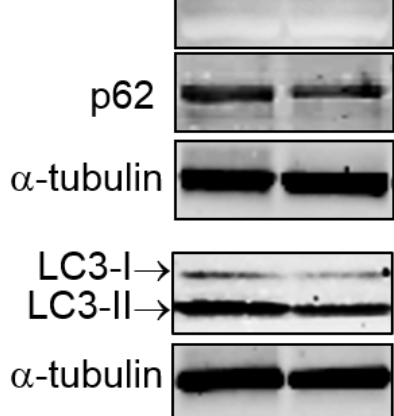

F.

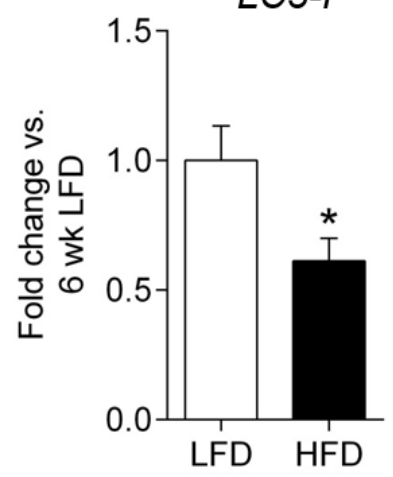

D.
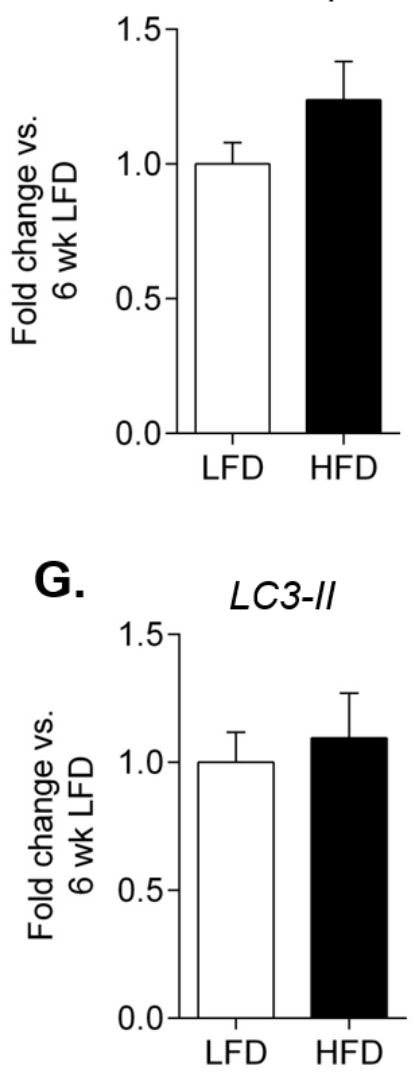

E.

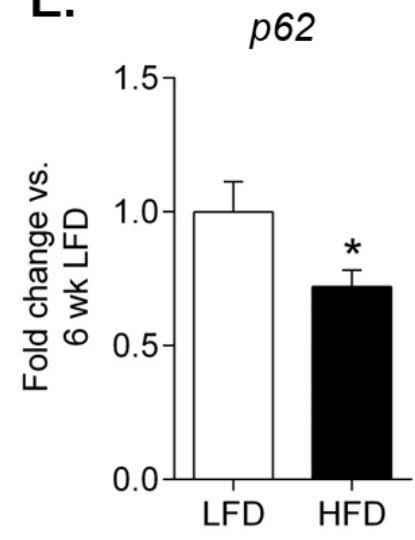


Figure 10: Evidence for activation of mitophagy in WAT of obese mice. Immunoblot analysis of markers of mitophagy and autophagy: (A) Western blots of Parkin and Pink 1 in adipose tissues from mice fed a LFD or HFD for 6 weeks (left panels) or 12 weeks (right panels); and (B) Quantification of Parkin and Pink1 abundance from panel E. $n=4$ per group; ${ }^{\star} p<0.05$ vs. LFD. $n=4$ per group; (C) Representative Western blots of ubiquitinated proteins, p62, and LC3 in mice fed a LFD or HFD for 6 weeks. (D-H) Quantification of protein-ubiquitin abundance, p62, LC3-I, LC3-II abundance, and LC3-II/LC3-I ratio. $\mathrm{n}=10$ per group; ${ }^{*} p<0.05$ vs. LFD. 


\section{Discussion}

The results of this study demonstrate coordinated restructuring of adipose tissue metabolism during adipocyte hypertrophy. Using metabolomics analysis, we identified several HFD-induced changes in key metabolites involved in energy, carbohydrate, nucleotide, lipid and amino acid metabolism. Importantly, these changes were relatively independent of inflammatory cell infiltration, and the data were corrected for adipocyte content; both of which help eliminate confounding features related to tissue and adipocyte composition. Our analysis showed an early decrease in citrate synthase activity that coincided with reduced expression of Pgc1a, even though mitochondrial abundance was not affected. Ultrastructural and immunological evidence suggested that whitening of the adipose tissue, evident after 12 weeks of HFD, may also be related to autophagy, which appears to occur early after adipocytes attain near maximum size. Taken together, these results reveal that conditions of nutrient excess promote progressive metabolic remodeling, decreased capacity for mitochondrial biogenesis, and potential activation of the autophagic program in WAT.

The major goal of this study was to understand how HFD affects adipose tissue biology and metabolism. This is important because key metabolic changes that occur during adipocyte hypertrophy could be targets for anti-obesity or insulin-sensitizing therapies. We found that 6 weeks of HF feeding led to a profound increase in fat mass, which was accompanied by a decrease in systemic $\mathrm{VO}_{2}, \mathrm{VCO}_{2}$ and $\mathrm{RER}$, and insulin resistance. Although adipose tissue inflammation is evident by the $10^{\text {th }}-12^{\text {th }}$ week of $\operatorname{HFD}(116,117)$, we find no 
significant increase in inflammatory cell infiltration with 6 weeks of HFD (e.g., see Figure 3 and $(109,117))$. This lack of extensive tissue infiltration by energetic inflammatory cells allowed us to examine HFD-induced changes specific to the adipose tissue.

Moreover, in our metabolic measurements, we accounted for changes in the composition of the adipocytes themselves. Because white adipocytes expand by storing triglycerides in the central lipid droplet, an equivalent tissue mass of WAT from a HF-fed mouse comprises fewer cells (and more triglyceride) than adipose tissue from a LF-fed mouse. Thus, normalization of metabolite levels by wet weight alone could lead to erroneous conclusions. An example of this is shown in Figs. 4 and 6, where uncorrected values indicate that most metabolites are decreased in WAT from HF-fed mice (Figure 4E, inset) and that mitochondrial OCR is diminished in adipocytes from the obese mice (Figure 7A\&B). Correcting for protein content in samples from HF-fed mice normalized the distribution of metabolic changes and rectified the leftward skew caused by decreased adipocyte content. Similarly, when values were normalized for protein content (rather than mg wet weight), there was no difference in tissue oxygen consumption between explants from LFD and HFD groups. This indicates that, with 6 weeks of HFD, there was no change in basal mitochondrial oxygen consumption per adipocyte.

Our metabolic profiling indicates that HFD affects several metabolic pathways. In mice placed on HFD for 6 weeks, we found significant changes in lipid, amino acid, energy, nucleotide, and carbohydrate metabolism. Higher levels 
of several fatty acids and lipid metabolites belonging to the linoleic acid metabolism subfamily were observed in WAT from HF-fed mice. In this tissue, there was also a higher abundance of arachidonic acid as well as metabolites involved in arachidonic acid synthesis, including linolenic acid and dihomolinolenic acid, which indicates a coordinated upregulation of this pathway. WAT from HF-fed mice also contained higher levels of the eicosanoid 12hydroxyeicosatetraenoic acid (12-HETE), which is formed from arachidonate by 12/15-lipoxygenase (12/15-LO). Interestingly, 12/15-LO is upregulated in white adipocytes of both HF-fed C57BL/6J mice (118) as well as Zucker obese rats (119). Thus, upregulation of 12/15-LO and increased production of its product (12-HETE) are consistent features of hypertropic WAT. This may be particularly significant in mediating adipose tissue dysfunction because two separate studies have shown that genetic deletion of 12/15-LO prevents HFD-induced adipose tissue inflammation and insulin resistance $(120,121)$.

The elevated levels of glycerophosphoethanolamine (GPEA) in WAT from obese mice could be due to an increase in the breakdown of phosphatidylethanolamine or a decrease in its rate of hydrolysis. Previous studies have shown that GPEA is hydrolyzed by enzymes such as glycerophosphodiester phosphodiesterase to form glycerol-3-phosphate (G3P) (122), which is required for triglyceride synthesis and thus would likely be in high demand in expanding adipocytes (123). Glycerophosphorylcholine (GPC), which was elevated in WAT from HF-fed mice, could also be utilized to form G3P. However, a major function of GPC in the cell is to act as an organic osmolyte 
(124), suggesting that there might be an increase in osmotic stress in hypertrophic adipocytes. This view is supported by our observation that the levels of several major osmolytes such as taurine, hypotaurine and creatine were higher in WAT from obese mice. Changes in osmolality could trigger a form of cell death called pyroptosis (125), which has been linked with inflammasome activity (126). Interestingly, the NLRP3 inflammasome is activated by cholesterol crystals, and, consistent with previous observations in hypertrophied adipocytes $(127,128)$, our analyses also showed an elevation of cholesterol in adipose tissue of obese mice. Collectively, these observations suggest an early increase in key metabolic instigators that could contribute to osmotic stress and inflammation in adipose tissue.

The HFD-induced increase in stearoyl sphingomyelin could further prime adipocytes for inflammation and metabolic dysfunction. Sphingomyelin (SM; d18:1/18:0)—which in humans is the only membrane phospholipid not derived from glycerol-is a type of sphingolipid found in cell membranes that consists of oleic acid attached to the $\mathrm{C} 1$ position and stearic acid attached to the $\mathrm{C} 2$ position. Genetic deletion of enzymes involved in sphingomyelin synthesis has been shown to protect against diet-induced obesity and insulin resistance (129, 130), and the breakdown of sphingomyelin could yield significant amounts of ceramide, which inhibits insulin signaling (131). Hence, the elevated levels of sphingomyelin observed in our study could prime adipocytes to release significant amounts of ceramide, which has been shown to be increased by $300 \%$ in both the plasma and the adipose tissue by HFD (132). Although 
ceramide levels were not measured in our analyses, we did observe a decrease in Pgc1a expression and a decrease in citrate synthase activity, both of which can be regulated by ceramide $(133,134)$. However, further studies are required to determine whether changes in mitochondrial biogenetic capacity and citrate synthase activity are related to high levels of stearoyl sphingomyelin.

Our metabolomic data suggest significant changes in intermediary metabolism related to energy expenditure and fat storage. We found that many carnitine derivatives were increased in WAT from HF-fed mice. These include acetylcarnitine, proprionylcarnitine, and deoxycarnitine; although 3dehydrocarnitine, an intermediate in carnitine degradation, was diminished. These changes suggest the presence of a "flooded" pool of adipocyte metabolites, which could be used for either energy provision, or, more likely, as a temporary repository for substrates for fatty acid synthesis. In other tissues such as the heart, acetylcarnitine functions as a buffer of the acetyl CoA pool, although it could be used as an energetic substrate as well (135). Similarly, the propionylcarnitine could buffer the propionyl CoA pool (136). Thus, it appears that in expanding adipocytes short-chain acylcarnitine pools could help buffer excess carbon until it is assimilated into fatty acids for triglyceride storage.

The observations that tissue levels of BCAAs such as leucine and valine were decreased, while propionylcarnitine and succinate levels were increased with HFD, suggest an increase in BCAA catabolism. Catabolism of BCAAs results in the formation of both acetyl CoA and succinyl CoA, the latter can be converted to succinate, which in our analysis was elevated in WAT from HF-fed 
mice. Thus, it is likely that in hypertrophic adipocytes there is increased utilization of BCAAs. In contrast to the decrease in BCAAs, we found that levels of Gln and Glu were increased with HFD. This increase may be particularly important, given the multifaceted uses of glutamine, e.g., in anaplerotic metabolism, as a nitrogen donor in anabolic processes, as well as a precursor for glutathione synthesis $(137,138)$. In addition, Gln and Asp are required for synthesis of several nucleotides such as IMP, UMP and CMP, all of which were increased in these studies. Interestingly, adipose tissue is a major site of glutamine synthesis, and its uptake can be regulated by changes in osmolality as well as by insulin (139).

Although there is a clear relationship between amino acids, insulin resistance and obesity in animal models and humans (140-142), the mechanistic basis of this link is less clear. BCAA metabolism in adipose tissue modulates plasma BCAA levels (143), and, interestingly, increasing circulating BCAAs in mice by preventing BCAA catabolism prevents diet-induced obesity and insulin resistance in mice (144), whereas feeding BCAAs to HF-fed rats increases insulin resistance (142). While further studies are required to test the full significance of such metabolic changes in BCAAs and other amino acid pathways, it is possible that changes in amino acid catabolism and downstream metabolites have novel roles in adipose tissue biology such as the regulating lipid storage and release. This possibility is supported by a recent report showing that succinate, which can be derived from BCAA breakdown, inhibits lipolysis by activating Gpr91 (a succinate receptor) (145). 
Metabolites related with glucose metabolism were also markedly affected by HFD. We found a nearly $50 \%$ decrease in the abundance of $1,5-\mathrm{AG}$ in WAT samples from HF-fed mice. Plasma 1,5-AG is a validated marker of short-term glycemic control and has been suggested to be in equilibrium in tissue and plasma pools $(113,114)$. Hence, even though 6 weeks of HFD does not appear to significantly affect fasting blood glucose levels (103), the decreased abundance of 1,5-AG is consistent with systemic insulin resistance and poor glycemic control (109). With respect to glycolysis, glucose-6-phosphate was decreased, and adipose tissue glucose was increased, suggesting that hexokinase might be a major rate-limiting step in the provision of glucose carbons required for triglyceride synthesis. Further evidence of changes in glucose metabolism is shown by diminished pdk4 expression, which regulates pyruvate dehydrogenase activity. This decrease in $p d k 4$ could potentially promote glyceroneogenic formation of G3P (146) via cataplerosis (147).

Interestingly, while we observed no change in basal mitochondrial OCR when corrected for protein content, WAT from HF-fed mice showed an increased response to FCCP. We speculate that once metabolic restraint due to $\Delta p$ is lifted (by addition of uncoupler), the increased substrate available for oxidative phosphorylation, e.g., succinate, malate, glutamate, short-chain acylcarnitines, results in increased rates of oxygen consumption. Also, in datasets corrected for protein content, there was a trend toward an increased rate of nonmitochondrial oxygen consumption, which might suggest an increase in adipocyte superoxide production due to cytosolic oxidase activity $(115,148)$. 
This possibility is consistent with previous studies showing an increase in oxidative stress in adipose tissue of obese mice, which is predominantly caused by an increase in NADPH oxidase and down-regulation of antioxidant enzymes (149-153).

Further support for coordinated remodeling of mitochondrial and cytosolic metabolism is provided by the observation that expression of Cox7a1 was increased in the adipose tissue of obese mice. A similar increase in Cox7a1 gene has been shown in the WAT of fattening cattle (154), although the implications of this increase are currently unclear. Cox7a1 is considered to be a heart- and muscle-specific subunit of cytochrome oxidase, but it is also present in brown adipose tissue; these tissues are highly metabolic organs capable of utilizing large amounts of substrate. Thus, it is possible that the increase in Cox7a1 may be an adaptive response to dissipate excessive energy in the adipocyte. The decrease in citrate synthase activity, which provides additional evidence for remodeling of mitochondrial metabolism during adipocyte hypertrophy, is similar to that observed in $d b / d b$ mice, where citrate synthase activity is diminished remarkably with no change in mtDNA/nDNA (155). Why citrate synthase decreases in expanding adipocytes is currently unclear; however, it could be an adaptation to prevent excessive influx of carbon into the Krebs cycle, which would be consistent with the view that during obesity there is an overall shift in metabolism from energy provision to lipid storage.

The notion that HFD remodels mitochondrial metabolism in adipocytes is further supported by diminished mitochondrial biogenetic capacity and later 
decreases in electron transport chain protein abundance. With of 6 weeks of HFD, mitochondrial abundance per se was not affected; however, we did find a significant decrease in Pgc1a expression, which was followed by loss of mitochondrial protein abundance after 12 weeks of HFD. This relatively late decrease in mitochondrial protein abundance may be due also to early changes in mitochondrial metabolism that promote removal of mitochondria by autophagy. Autophagy has been shown previously to be increased in adipocytes from obese humans and mice (156-158). Consistent with those studies, our biochemical results show an increase in the LC3-II/LC3-I ratio and a decrease in p62, the combination of which would appear to be consistent with an increase in autophagic flux $(159,160)$. Ultrastructural data showing autophagosomes and vacuolated structures containing electron-dense material further suggest changes in autophagy consistent with its activation. A limitation of this study, however, is that autophagic flux was not directly measured, making it difficult to ascribe with certainty directional changes to the autophagy pathway. Nevertheless, an activation of autophagy in adipose tissue would be expected to decrease the abundance of both LC3-I and p62 (161-164), which is consistent with our results. Hence, our cumulative data are supported by evidence in the literature and suggest that autophagy is activated by HFD. While we cannot clearly distinguish whether mitochondria are removed by autophagy, the increase in key proteins involved in mitophagy - Parkin and Pink1 $(115,165)$ - would be consistent with a significant role of mitophagy in mediating mitochondrial remodeling and turnover in the WAT of obese mice. 
In summary, in this study we identified key metabolic changes that occur during WAT expansion. These coordinated changes occur before the infiltration of inflammatory cells and include: loss of mitochondrial biogenetic capacity; dysregulation of lipid, amino acid, glucose, nucleotide and amino acid metabolism; changes in mitochondrial gene expression and protein abundance; and evidence of alterations in autophagy and possibly mitophagy. Our results are consistent with the view that constant nutrient excess shifts adipocyte metabolic activity from energy provision to lipid storage. We speculate that the decrease in mitochondrial biogenetic capacity and later removal of mitochondria may also be a response to limit excessive lipid oxidation and oxidative stress and to promote fat storage. Further studies are required to assess the significance of each of the metabolic alterations and to test whether therapeutically targeting these pathways is a gainful strategy for preventing obesity and its unfortunate metabolic consequences. 


\section{CHAPTER 3}

\section{REGULATION OF ADIPOSE TISSUE STEM CELL POPULATIONS BY OBESITY}

\section{Introduction}

Storing lipid in the form of esterified fat is the primary function of white adipose tissue. Adipose tissue can expand to increase lipid uptake in two ways, via adipocyte hypertrophy or hyperplasia $(16,57)$. Adipocytes can hypertrophy to accommodate massive lipid loads; however when maximum expansion is achieved, adipocytes begin to die by way of necrosis leading to an increase in adipose tissue inflammation, hypoxia, and insulin resistance $(56,57,166,167)$. Adipose tissue expansion has been shown to occur in this manner with prolonged consumption of HFD (15). Adipocyte progenitor cells (APCs), which can give rise to mature adipocytes, have been shown to proliferate and differentiate allowing for expansion of adipose tissue by way of hyperplasia. This type of expansion appears to maintain metabolic homeostasis, suggesting hyperplasia is a more metabolically favorable form of adipose tissue remodeling $(37,66)$.

Putative APCs have been described as cells expressing CD24 and CD29 and can be isolated from both bone marrow and adipose tissue. Rodeheffer, et al. showed that when these cells, when transplanted into insulin resistant, 
lipodystrophic mice, proliferated, differentiated, and gave rise to a functional adipose organ capable of storing lipid. The development of this tissue improved insulin sensitivity in the transplanted animals (37). While APCs are required to improve the fat storage capacity of adipose tissue, several types of supporting cells would be required. For example, to sustain proper tissue oxygenation in expanding tissue and to nurture newly formed, APC-derived adipocytes, the vasculature network would need to grow in tandem with the expanding adipose organ. Thus, endothelial progenitor cells (EPCs), which have been shown to repair damaged endothelium and support the formation of new blood vessels in vivo (168-171), would likely be in high demand during periods of increased fat storage. In mice, Sca-1+ Flk1+ cells have been identified as EPCs and are believed to have the capacity to differentiate into mature vascular networks, promote vascular stability, or support angiogenesis $(71,76)$.

Chronic adipose tissue inflammation and hypoxia are known to accompany the development of obesity, and these processes, in addition to metabolic changes $(15,55,56,172)$, could negatively affect adipose tissue stem cell populations. Nevertheless, the impact of obesity on these cell progenitor cell populations is not clear. Because both an adequate amount of adipocytes and oxygen availability are necessary to remodel adipose tissue, we hypothesize that the balance of CD24+ CD29+ APCs and Sca-1+Flk1+ EPCs is crucial for favorable adipose tissue expansion. A better understanding the changes that occur in progenitor cell populations of adipose tissue could be beneficial in determining new treatments for obesity. 
To track the progenitor cell populations of interest, we performed a bone marrow transplant, replacing bone marrow of wild-type mice with GFP positive bone marrow cells. Donor bone marrow cells and progeny expressed GFP which was detectable in the stromal vascular fraction (SVF). Analysis of SVF using flow cytometry allowed us to quantify changes in the APC and EPC populations after 12 weeks of HFD as well as to determine the origin of each cell type. Results from this study show that obesity increases the ratio of APCs to EPCs in adipose tissue. Moreover, we find that more than 96\% APCs are bone marrow-derived, unlike EPCs which appear to be primarily tissue-resident. These data provide fundamental information regarding the source of stem cells in adipose tissue and suggest that changes in the number or function of angiogenic and adipogenic progenitor cell populations may be an important feature of adipose tissue remodeling.

\section{Experimental procedures}

Animal Studies: All procedures were approved by the University of Louisville Institutional Animal Care and Use Committee. All mice used in these studies were purchased from The Jackson Laboratory (Bar Harbor, ME).

Four week old C57BL/6J (wild-type; WT) mice were acclimated to the animal facility for one week before being subjected to 10 minutes of total body irradiation at a dosage of 950 cGy by way of a cesium source (173). Twenty-four hours after irradiation, bone marrow from 8 week old male mice expressing "enhanced" green fluorescent protein (EGFP; The Jackson Laboratory, \#006567) 
was isolated and used to transplant irradiated WT mice via a single retro-orbital injection. Approximately, $1 \times 10^{7}$ bone marrow stromal cells were injected into each mouse using $100 \mu \mathrm{l}$ sterile PBS as the vehicle (Scheme 2). Five weeks after transplantation, at 9 weeks of age, bone marrow transplanted (BMT) mice were placed on either a 10\% low fat diet (LFD; Research Diets, Inc., \#D12450B) or a $60 \%$ high fat diet (HFD; Research Diets Inc., \#D12492) for 12 weeks. Water and diet were provided ad libitum and body weights were recorded weekly. To assess proliferative capacity of adipose tissue-resident stem cells, $33 \mathrm{mg} / \mathrm{kg} / \mathrm{day}$ bromodeoxyuridine (BrdU) was administered via i.p. injection each day for 7 days prior to euthanasia.

Separate cohorts of WT mice, not subjected to BMT, were maintained on LFD or HFD for 12 weeks. These animals received water and food ad libitum and body weights were measured on a weekly basis for the duration of the study. At the time of euthanasia, wet organ weights were measured and recorded to assess potential differences between study groups.

Body composition measurement: Lean mass, fat mass, percent body fat, and total body mass were measured by Dual X-ray Absorptiometry (DEXAscan) paired with a mouse densitometer (PIXImus; Lunar, Madison, WI). For this measurement, the body weight of each mouse was taken prior to the scan and animals were anesthetized with isofluorane gas for the duration of the DEXAscan. Measurements for each parameter were recorded and images of each animal were collected. 
Activity assessment: Total activity level, ambulatory counts, fine movements, and respiratory exchange ratio (RER) were measured using a physiological/metabolic cage system (TSE PhenoMaster System; Bad Homberg, Germany). The mice were singly housed throughout the entire assessment. Cages were located in a temperature controlled room and when the light cycle was also regulated to allow for 12 hours of light and 12 hours of darkness.

Glucose and Insulin Tolerance Tests: After a $6 \mathrm{~h}$ fast, a bolus of glucose (1.0 $\mathrm{mg} / \mathrm{g}$ in sterile saline) was injected i.p. and blood glucose measurements were recorded over the course of $2 \mathrm{~h}$ using blood collected from the tail vein. For insulin tolerance tests, a bolus of insulin (1.5 U/kg Humulin R in sterile saline) was injected i.p. into non-fasted mice and blood glucose was measured over a 2 $h$ period.

Isolation of adipose stromal vascular fraction: The stromal vascular fraction (SVF) was isolated from the visceral and subcutaneous adipose depots. The adipose tissue was finely minced and digested in collagenase $(500 \mathrm{CDU} / \mathrm{mL}$ $\mathrm{PBS} / \mathrm{HEPES}$ ) at $37^{\circ} \mathrm{C}$ for $25 \mathrm{~min}$. The mixture was then filtered to remove any particles larger than $100 \mu \mathrm{m}$ and washed several times to remove excess collagenase from the cell fraction.

Analysis of adipose tissue by flow cytometry: SVF cells were isolated as previously described in this chapter and used for flow cytometric analysis. The Fc receptor was blocked for 10 min at $4^{\circ} \mathrm{C}$ to prevent non-specific antibody binding. The diverse SVF population was then stained for $30 \mathrm{~min}$ at $4^{\circ} \mathrm{C}$ with antibodies to detect each of the following cell populations: adipocyte progenitor cells (Sca-1, 
CD24, CD29, CD90.2), endothelial progenitor cells (Sca-1, Flk1), leukocytes (CD45), monocytes (CD14), macrophages (F4/80, CD11c, and CD301), T cells (CD3, CD4, and CD8), B cells (B220/CD45R), and granulocytes (GR-1). All antibodies (purchased from eBioscience or Biolegend) were conjugated to unique fluorochromes for detection in 2 or more distinct panels. Samples were analyzed using the BD LSR II and FACSDiva Software. Experiment files were exported and further analyzed using the FlowJo analysis software.

WT, C57BL6/J mice from the Jackson Laboratory were used as bone marrow cell recipients for this study. Mice expressing EGFP were used as bone marrow cell donors. We chose to use these mice as donors so that we could identify cell origin. Since donor cells expressed GFP, any cell which also expressed GFP was deemed to be a bone marrow-derived cell. Therefore, GFPcells were deemed tissue resident cells.

Assessment of proliferation in adipose tissue: Immunofluorescent staining of adipose tissue sections was performed with aid from the Pathology CORE at the Diabetes and Obesity Center. Staining for transplanted cells, BrdU, and cell nuclei were done using primary antibodies against the following proteins: GFP, BrdU, and DAPI, respectively. Distinct fluorescent secondary antibodies were used to detect each of the primary antibodies. The Zeiss confocal microscope belonging to the Department of Molecular Cardiology at the University of Louisville was used to generate images of the fluorescent-stained adipose tissue sections. The Carl Zeiss ZEN 2012- blue edition was used to capture and analyze each of the confocal images. Cell expression of GFP, BrdU, and Dapi 
were quantified independently using the Nikon Elements-Basic Research software package. To determine proliferative capacity of bone marrow-derived cells, cells expressing both GFP and BrdU were also quantified.

Statistical Analyses: Statistical analyses were performed using the Prism GraphPad software. For direct comparison of two groups, Student's $t$ test was used. For comparison of multiple groups, one-way or two-way analysis of variance tests (ANOVA) were used with Bonferroni post-testing. Data are expressed as mean \pm standard error of the mean (SEM) unless otherwise noted. Significant differences between groups are marked on the graph with "*" denoting $p$ value $\leq 0.05$, "**" denoting $p$ value $\leq 0.01$, and "***" denoting $p$ value $\leq 0.0001$. 


\section{Scheme 2}

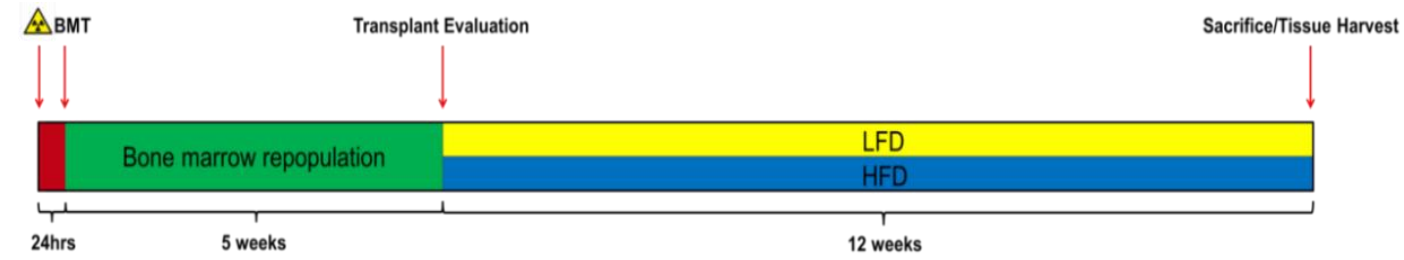

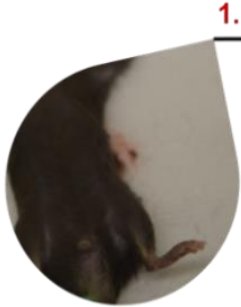

Stabilize animal

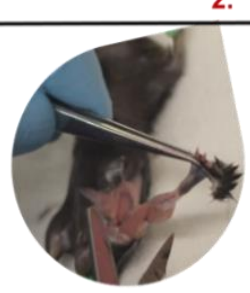

Remove tibia and femur from each of the hind limbs
2.

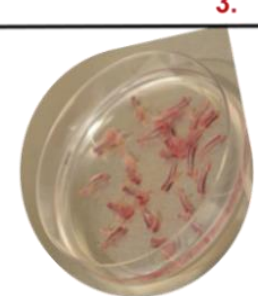

Detach skeletal muscle from tibia and femur.

3.
4.

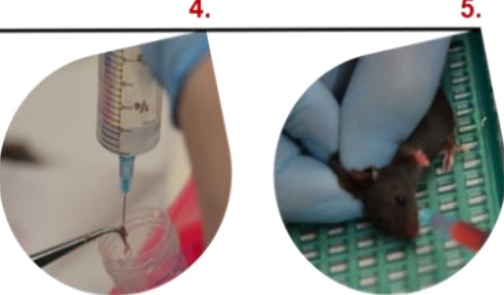

Flush each bone with PBS and centrifuge mixture to pellet cells.
Transplant BM cells via retro-orbital injection. 
Scheme 2: Bone marrow transplantation protocol. Bone marrow was isolated from both hind limbs of 8 week old, male EGFP donor mice. These cells were harvested in sterile isolation medium, washed, filtered and reconstituted in sterile PBS for transplantation. Anesthetized animals were then given bone marrow cells via retro-orbital injection. Mice were closely monitored for 10 days to ensure recovery from the transplant. During this time, mice were fed normal chow and given water, by way of water bottles, ad libitum. Five weeks post-transplantation, mice were fed a $10 \%$ low fat or $60 \%$ high fat diet for the duration of the study. 


\section{Results}

Adiposity is significantly increased after 12 weeks of high fat diet. Because 12 weeks of HFD has been suggested to promote maximal adipocyte hypertrophy and result in metabolic changes that promote a pre-diabetic state $(15,54,56)$, this model was used to elucidate the impact of obesity on adipose stem cells. For this, WT, C57BL6/J mice were fed either a 10\% LFD or $60 \%$ HFD ad libitum for 12 weeks. Total body mass and total weight gain were significantly increased with consumption of HFD (Figure 11A,B). After 12 weeks of feeding, body composition was measured using Dexascan (Figure 11C,D). Dexascan analysis showed 12 weeks of HFD increased total body mass by 1.7fold (Figure 11E). Lean mass, which accounts for muscle and bone mass, was unchanged (Figure 11F). However, total fat mass increased 2-fold (Figure 11G) and fat mass as a percentage of the total body mass increased 3.5-fold (Figure $11 \mathrm{H})$ after 12 weeks of HFD.

Consumption of high fat diet decreases activity. To examine the impact of obesity on metabolic and physiological activity, we placed obese and lean mice in metabolic cages for 24 hours. Beams along the bottom of the cage were used to determine the type, fine or ambulatory, and duration of movement. Each beam break was counted and the total number of beam breaks per 10 min interval was recorded and quantified (Figure 12). Twelve weeks of HFD decreased total movements 1.9-fold (Figure $12 \mathrm{~A}, \mathrm{~B})$. Ambulatory movement counts were diminished 2.1-fold with HFD (Figure $12 \mathrm{C}, \mathrm{D}$ ) and fine movements declined 1.5fold (Figure $12 \mathrm{E}, \mathrm{F})$. Respiratory exchange ratio was also decreased 
significantly, $0.9009 \pm 0.003135$ with LFD and $0.7712 \pm 0.001059$ with HFD (Figure $12 \mathrm{G}, \mathrm{H}$ ). 
Figure 11

A.

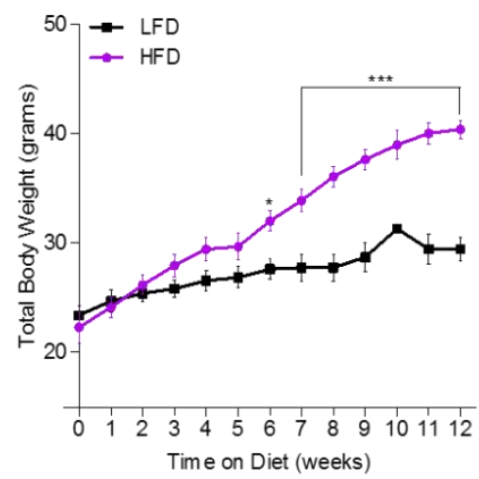

c.

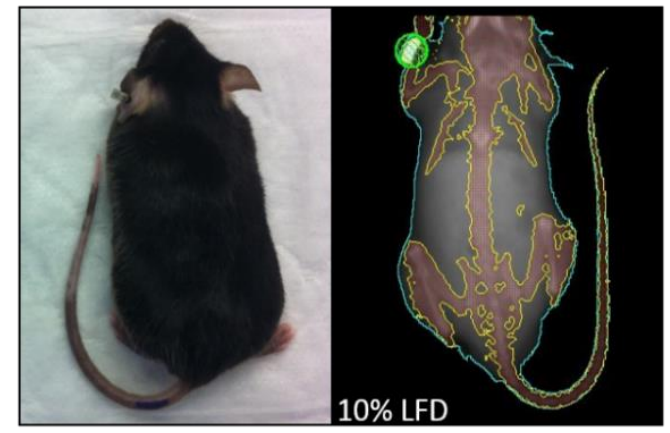

E.

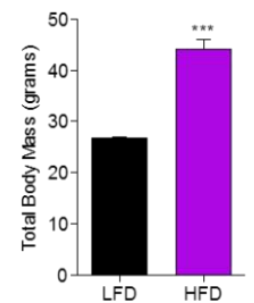

F.

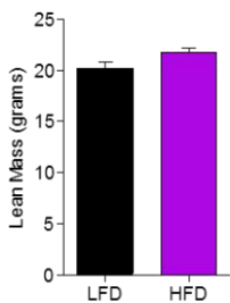

B.

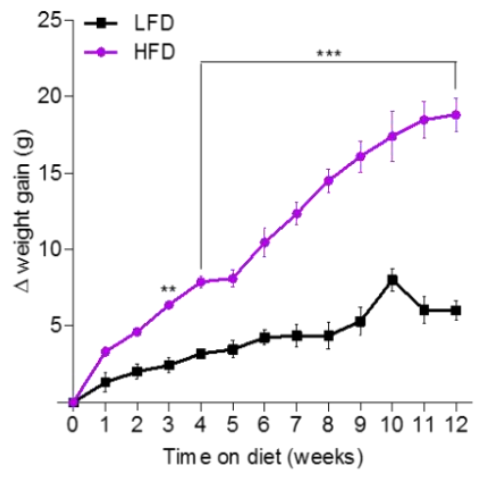

D.

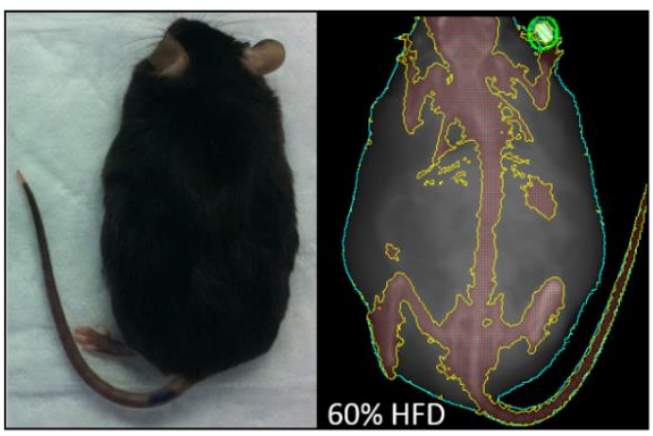

G.

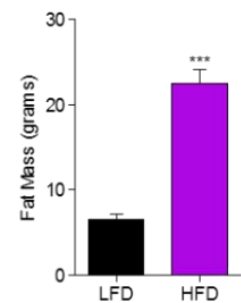

H.

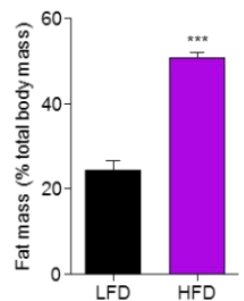


Figure 11. Adiposity increases significantly after consumption of a $60 \%$ fat diet for 12 weeks. Total body weight gain, (A), and delta body weight gain (B), are plotted in grams. Representative photographs and Dexascan images of mice fed a $10 \%$ low fat or $60 \%$ high fat diet for 12 weeks are provided above, $(\mathrm{C})$ and (D), respectively. Total body mass, $(E)$, lean mass, $(F)$, and fat mass, $(G)$, were quantified and presented in grams of tissue. Total fat mass is expressed as a percentage of the total body mass, $(\mathrm{H}) ; \mathrm{n}=4-6$ per group; ${ }^{*} p \leq 0.05,{ }^{* *} p \leq 0.01$, and ${ }^{* * *} p \leq 0.001$ vs. LFD. 
Figure 12

A.

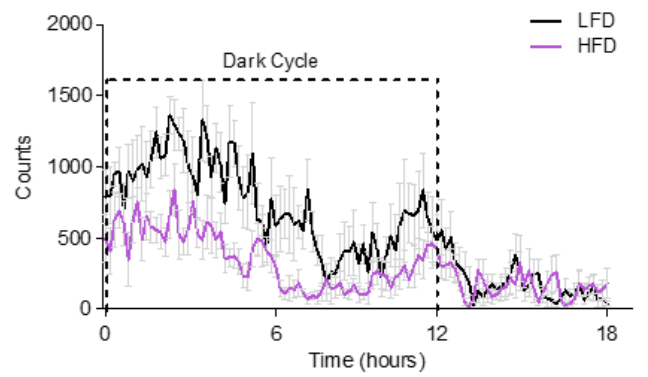

C.

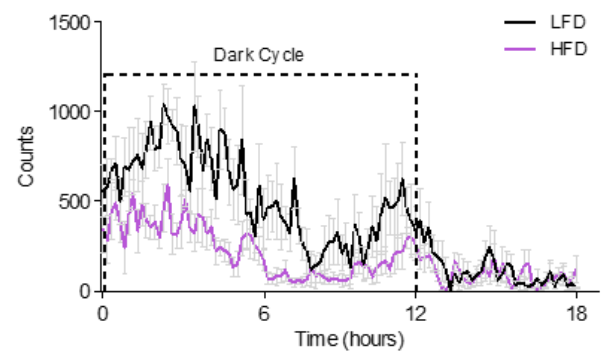

E.

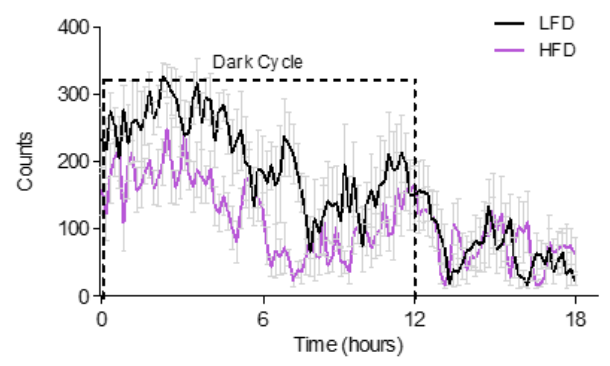

G.

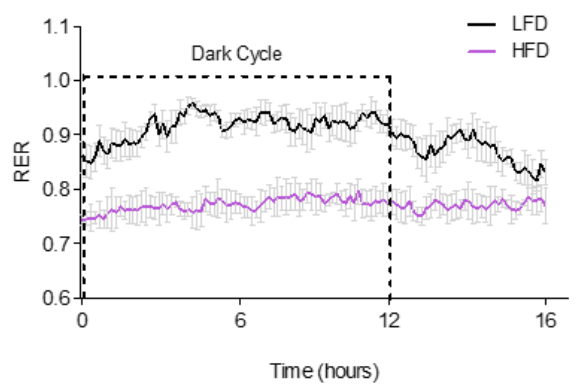

B.

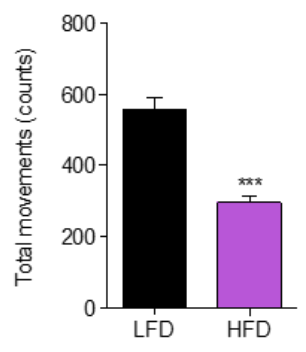

D.

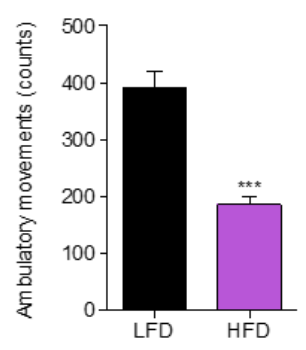

F.

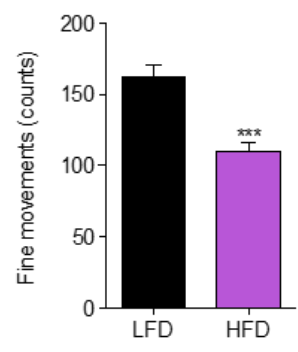

H.

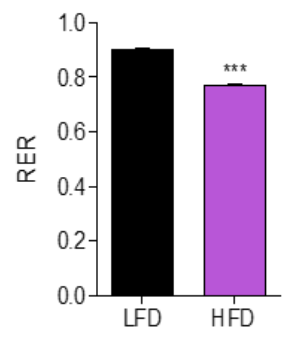


Figure 12: Consumption of high fat diet decreases activity. Male WT C57BL/6J mice were fed a low fat diet (LFD, 10\% kcal fat) or high fat diet (HFD, $60 \%$ kcal fat) for 12 weeks and the following measurements were recorded and means were quantified: Total activity level, (A) and (B); ambulatory counts, (C) and (D); fine movements, $(E)$ and $(F)$; and respiratory exchange ratio $(R E R),(G)$ and $(\mathrm{H}) . \mathrm{n}=6$ per group; ${ }^{* * *} p \leq 0.001$ vs. LFD. 
Whole body glucose tolerance is significantly reduced by long-term consumption of a $60 \%$ high fat diet for 12 weeks. Mice fed a HFD had elevated blood glucose levels following a glucose challenge and area under the curve was increased 1.6-fold above LFD group (Figure 13A,B). There was not a significant difference in insulin tolerance (Figure 13D). Together, these data suggest that glucose tolerance is impaired due to diet-induced obesity.

\section{Visceral adipose depot expands tremendously with 12 weeks of high fat}

feeding. To examine gross changes in size, wet tissue wets of several organs were measured after the establishment of obesity. In this case, both epididymal fat pads were harvested and weighed together to yield a total visceral adipose tissue measurement, vWAT. There was a marked, 5 -fold increase in the mass of the visceral adipose tissue of the mice fed a HFD (Figure 14). Since the visceral white adipose tissue was most significantly changed with obesity, we next assessed changes in the cell composition of visceral adipose tissue.

Total T cell and macrophage populations increase significantly in visceral adipose tissue with obesity. To examine the inflammatory status of the adipose tissue, F4/80 positive cells (macrophages) and CD3 positive cells (T cells) were examined in the stromal vascular fraction isolated from the visceral and subcutaneous adipose tissue. Only the visceral adipose tissue showed significant differences in the total number of inflammatory cells per gram tissue due to high fat feeding, furthering support for the argument that the visceral adipose tissue is most significantly affected by the development of diet-induced obesity in our model. There was a 2.9-fold increase in F4/80 positive macrophage cells and a 
1.9-fold increase in CD3 positive T cells in the visceral adipose tissue after 12 weeks of HFD (Figure 15A,C). 
Figure 13

A.

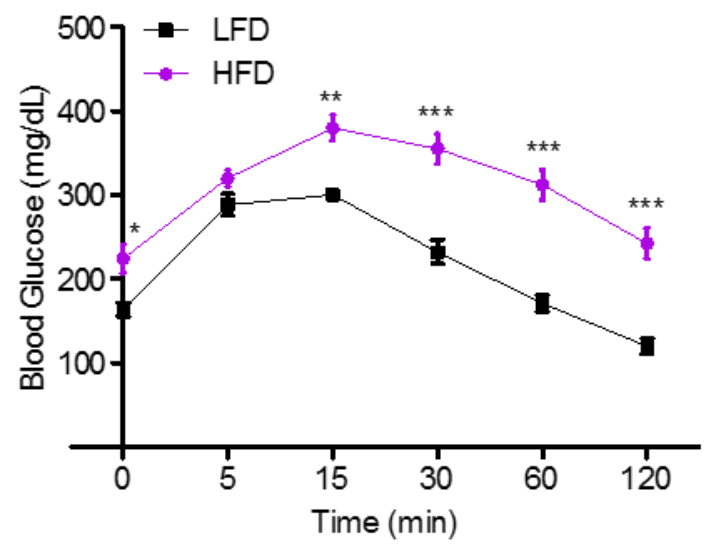

C.

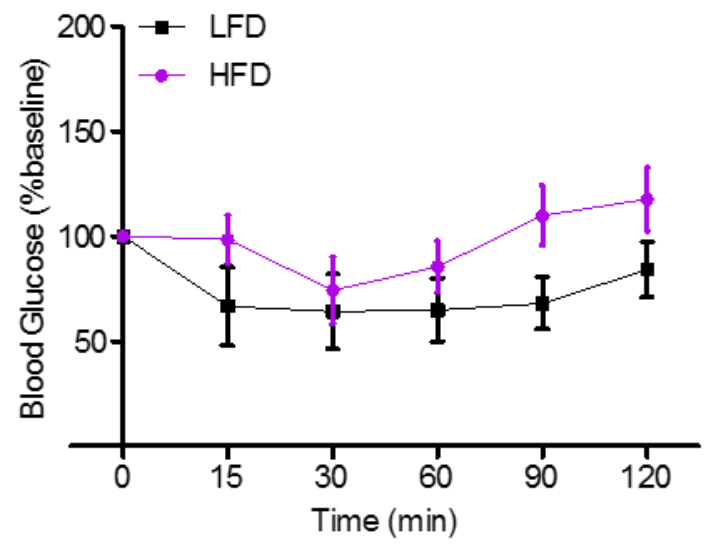

B.

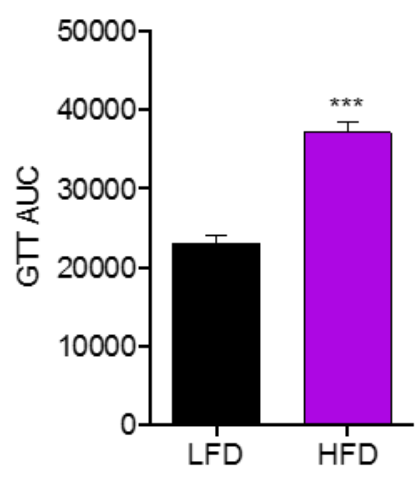

D.

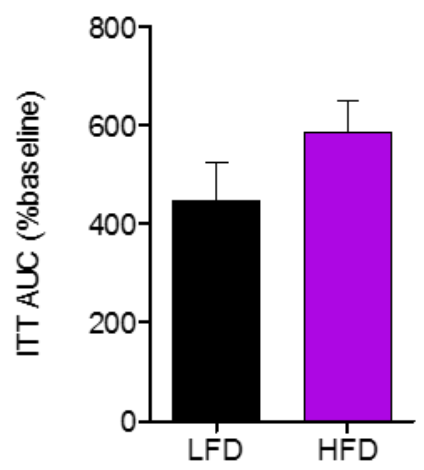


Figure 13. Measurements of insulin sensitivity in WT mice after 12 weeks of high fat diet. After 12 weeks of a LFD or HFD, a glucose tolerance test, (A), and insulin tolerance test, (C), was performed on a cohort of obese mice. Area under the curve for the glucose tolerance test was calculated and expressed as such in graph (B). Area under the curve was calculated and normalized to the baseline glucose measurement for the insulin tolerance test, graph (D). For each measurement, $\mathrm{n}=4$ per group; ${ }^{* *} p \leq 0.001$ vs. LFD. 
Figure 14

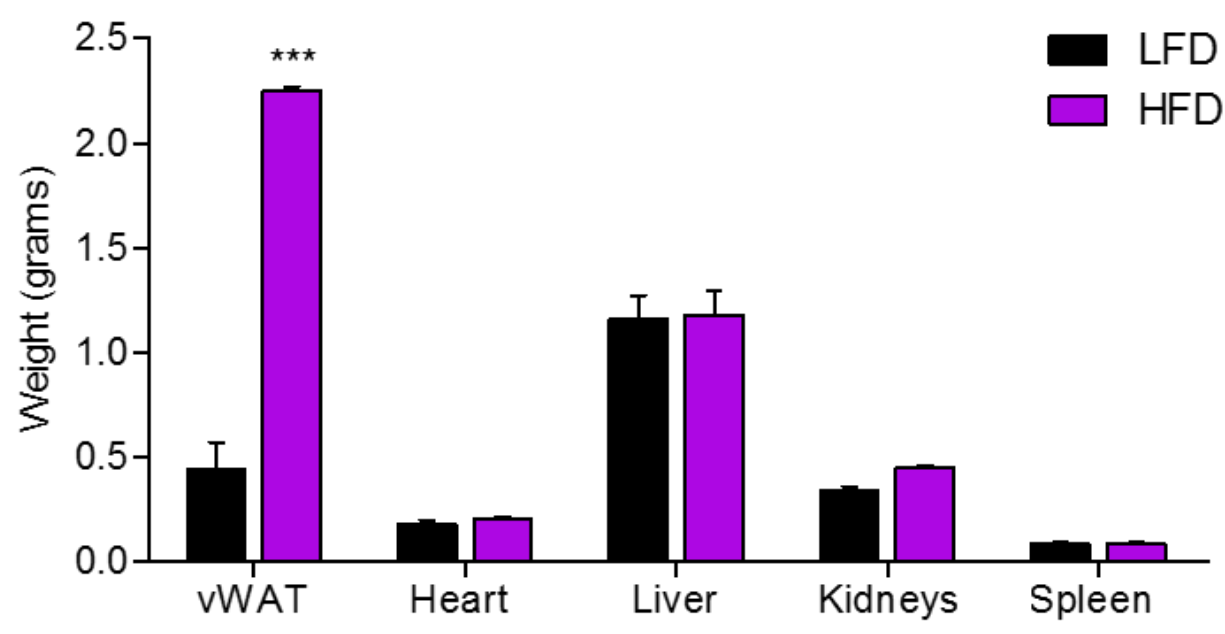


Figure 14. Wet tissue weight of organs harvested from animals fed LFD or HFD for 12 weeks. After 12 weeks of feeding, tissues were collected from each of the mice and the wet weights are quantified above (A); $n=6$ per group; ${ }^{* *} \mathrm{p} \leq 0.001$ vs. LFD. 
Figure 15

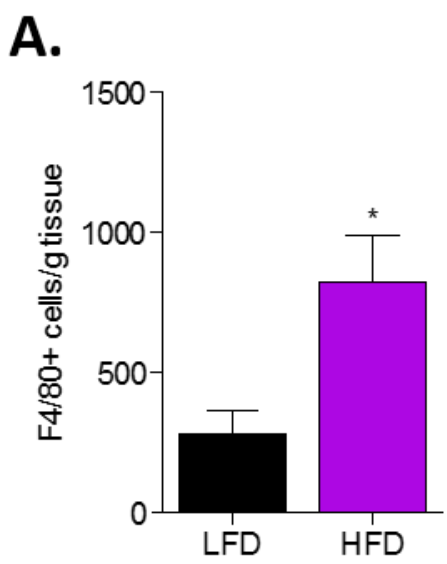

B.

C.

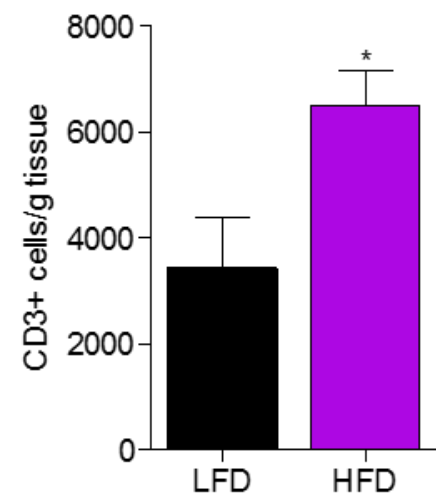

D.

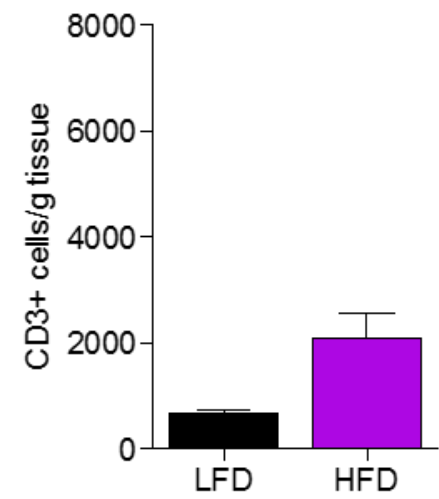


Figure 15. Quantification of F4/80+ and CD3+ cell populations in visceral and subcutaneous adipose tissue after consumption of $60 \%$ high fat diet for 12 weeks. The quantification for the total number of $F 4 / 80+$ macrophage cells per gram of visceral or subcutaneous adipose tissue are presented above; graphs $(A)$ and $(B)$, respectively. Quantification of the total CD3+ T cells per gram of visceral or subcutaneous adipose tissue is also included in the figure; (C) and (D), respectively; $n=7-8$ per group; ${ }^{*} \mathrm{p} \leq 0.05$ vs. LFD. 
Imbalance in progenitor cells pools in the visceral adipose tissue of obese mice. Adipocyte progenitor cells, APCs, and endothelial progenitor cells, EPCs, were identified as outlined in Scheme 3. To understand the diet-induced changes in these two populations, APCs and EPCs were identified in the stromal vascular fraction, SVF, using flow cytometry. Each SVF sample was stained with antibodies to detect cell surface antigens of interest. Based upon the expression of these antigens, the CD14- CD45- CD24+ CD29+ APCs and CD14- CD45Sca-1+ Flk1+ EPCs were identified and quantified (Figure 16A,B). Though there was no significant difference in the number cells per gram fat with HFD, the ratio of APCs to EPCs was increased more than 5-fold in the high fat fed group (Figure 16C).

\section{Adipocyte progenitor cells in visceral adipose tissue of obese mice are} bone marrow-derived. To determine the origin of the progenitor cell populations of interest, a bone marrow transplant was performed. Recipient mice were treated with a lethal dose of irradiation to eradicate all existing bone marrow cells. These cells were repopulated with GFP positive donor cells 24 hours after irradiation. To allow for proper repopulation, mice were monitored and maintained on normal chow for 5 weeks before they were placed on a low fat or high fat diet or 12 weeks. Bone marrow-derived, GFP positive APCs accounted for $>96 \%$ of all APCs identified in the visceral stromal vascular fraction of both low fat and high fat fed mice (Figure 17B,C). Conversely, $>80 \%$ of EPCs were GFP negative, indicating that most EPCs in the visceral adipose tissue stromal vascular fraction derive from a tissue-resident pool (Figure 17E,F). 
Proliferation of adipose tissue stromal cells is decreased after 12 weeks of HFD. BrdU was detected via histological analysis of paraffin-embedded visceral adipose tissue sections. The percentage of total DAPI positive cells expressing BrdU was decreased 1.48-fold HF-fed mice compared to mice fed LFD (Figure 18A). BrdU positivity of bone marrow-derived cells expressing GFP was not different between the groups (Figure 18B). These data suggest that proliferation of adipose tissue-resident stromal cells, whereas proliferation of bone-marrow derived cells is unaffected, by HFD.

\section{Reversion from 60\% HFD to 10\% LFD decreases adiposity and restores} insulin sensitivity. We established a model of obesity by feeding 8 week old C57BL6/J mice a $60 \%$ HFD for 12 weeks and then reverted the animals to a $10 \%$ LFD for 4 weeks to determine if weight loss improved insulin sensitivity in mice. The reverted cohort experienced a significant amount of weight loss and after 4 weeks of LFD, the percent weight gain measured in the reverted cohort was no longer different from that of the cohort fed a LFD for the entire 16 week period (Figure 19A). Area under the curve for the glucose and insulin tolerance tests was decreased significantly after reversion to LFD and was not significantly different from the LFD group (Figure 19D,E).

\section{Progenitor pools are increased after reversion of obese mice to a $10 \%$ low} fat diet. Adipocyte progenitor cells, APCs, and endothelial progenitor cells, EPCs, were identified as previously described. To examine changes in the progenitor cell populations with weight loss, obese, HFD group was compared to the reverted, HFD-LFD, group. The weight loss induced a 7.7-fold increase in 
APCs and a 4.4-fold increase in EPCs (Figure 20A,B). The ratio of APCs to EPCs was also increased 1.5-fold (Figure 20C) compared to the HFD fed mice. 
Scheme 3

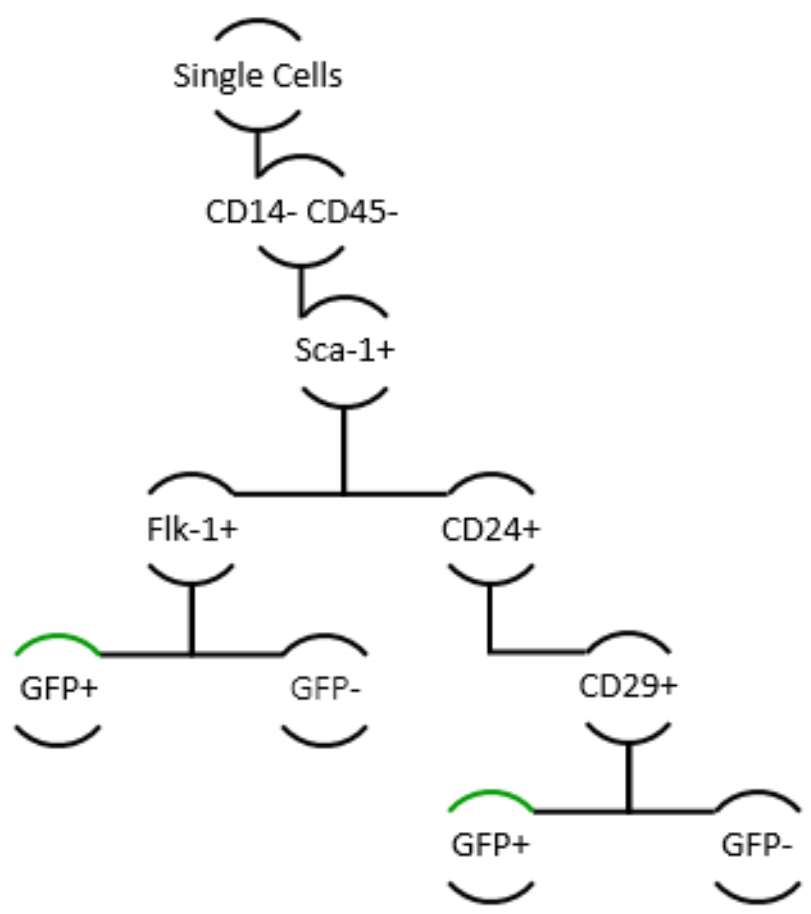


Scheme 3. Progenitor cell identification. The diagram above depicts the method used to discriminate progenitor cell populations from the heterogeneous stromal vascular fraction. The same hierarchy is used to identify progenitors in other heterogeneous depots, such as bone marrow or blood. 
Figure 16

A.

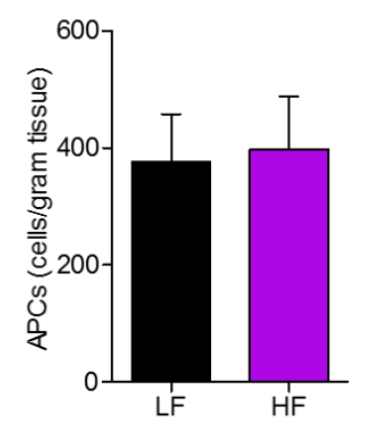

B.

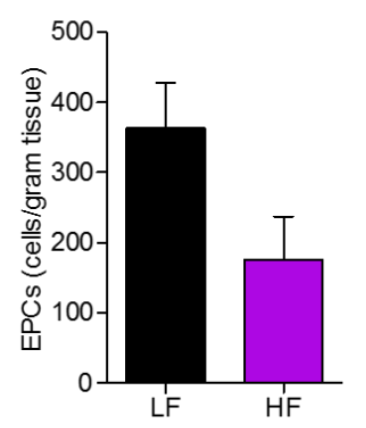

C.

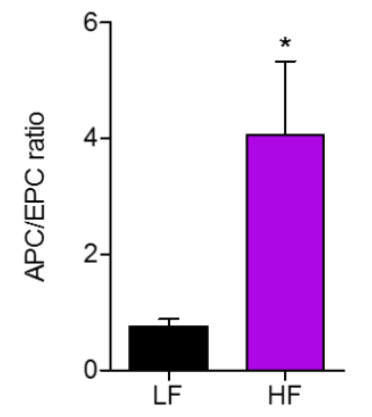


Figure 16. Quantification of adipocyte progenitor cell, APC, and endothelial progenitor cell, EPC, populations of visceral adipose tissue after consumption of $60 \%$ high fat diet for 12 weeks. The quantification for the total number of CD14- CD45- Sca-1+CD24+ CD29+ APCs per gram of visceral adipose tissue are presented above in graph (A). Quantification of the total CD14- CD45- Sca-1+ Flk-1 + EPCs per gram of visceral adipose tissue is also included in graph (B). The ratio of APCs to EPCs is presented in graph (C); $n=6$ per group, ${ }^{*} \mathrm{p} \leq 0.05$ vs. LFD. 
Figure 17

A.

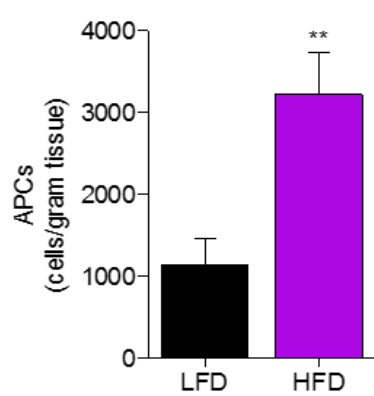

D.

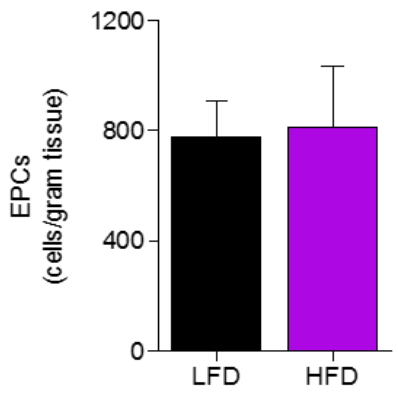

G.

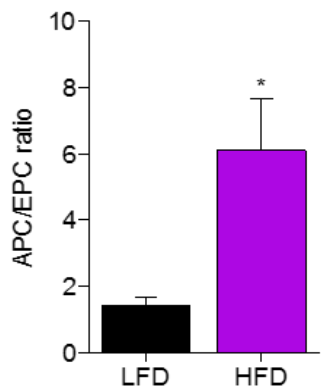

B.

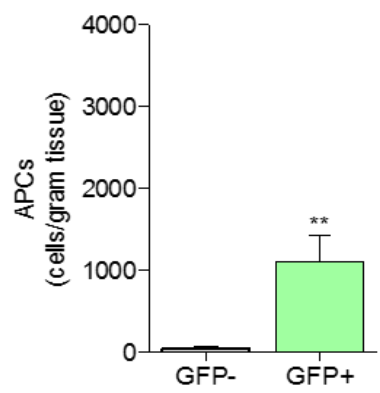

E.

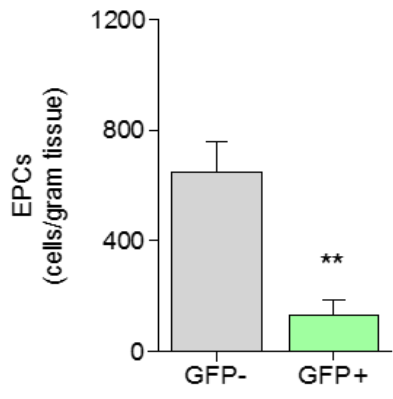

C.

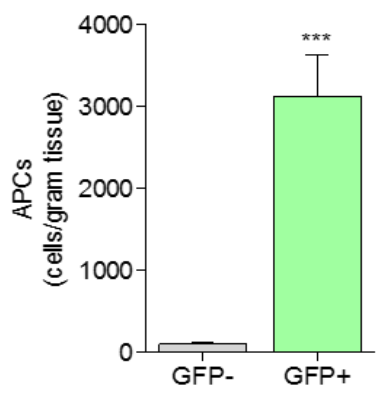

F.

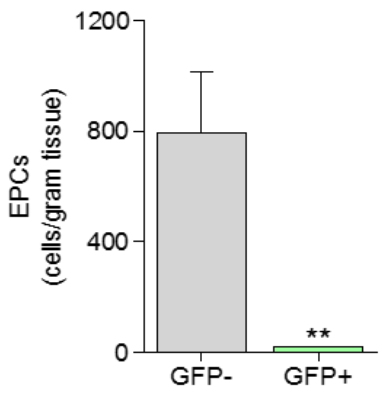


Figure 17. Quantification of adipocyte progenitor cell, APCs, and endothelial progenitor cell, EPC, populations of visceral adipose tissue after bone marrow repopulation and consumption of $60 \%$ high fat diet for 12 weeks. After bone marrow transplantation with GFP+ stromal cells, mice were placed on $10 \%$ LFD or $60 \%$ HFD for 12 weeks. The quantification for the total number of CD14- CD45- Sca-1+ CD24+CD29+ APCs per gram of visceral adipose tissue are presented above in graph (A). GFP+ and GFP- APC populations of in the LF, (B), and HF (C), cohorts are quantified as cells per gram tissue. Total CD14- CD45- Sca-1+ Flk-1+ EPCs in the visceral adipose tissue of bone marrow transplanted animals is presented as cells per gram tissue, (D). GFP+ and GFP- EPC populations of in the LF, $(E)$, and HF (F), cohorts are quantified as cells per gram tissue. APCs to EPCs ratio is provided in graph (G); $n=7-8$ per group; ${ }^{*} p \leq 0.05,{ }^{* *} p \leq 0.01$, and ${ }^{* * *} p \leq 0.001$ vs. LFD. 
Figure 18

A.

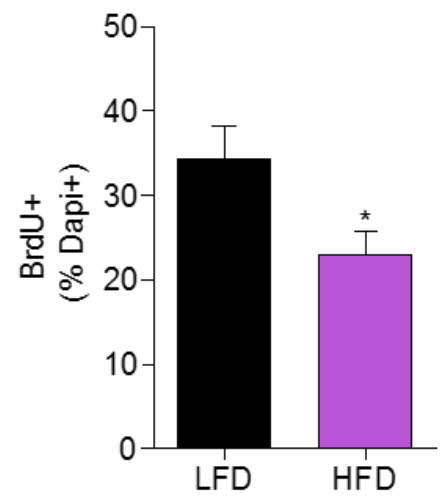

B.

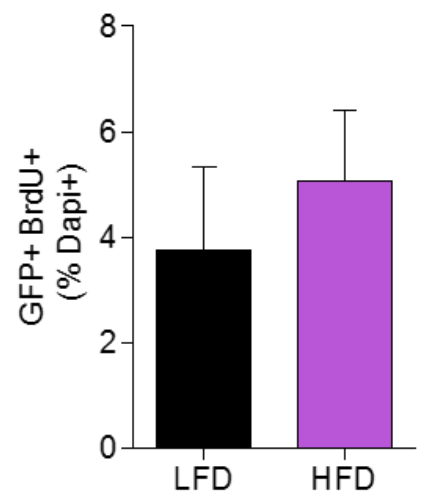


Figure 18. Quantification of BrdU in adipose tissue after 12 weeks of LFD or HFD. Quantification of the total percentage of BrdU+ cells are presented in graph (A). GFP+ BrdU+ cells in the visceral adipose tissue are also presented as a percentage of the total Dapi+ cell population (B); $n=4$ mice/group (4-5 images/animal) for LFD and $n=4$ mice/group (5-10 images/animal) for HFD; ${ }^{*} p \leq 0.05$ vs. LFD. 
Figure 19

A.

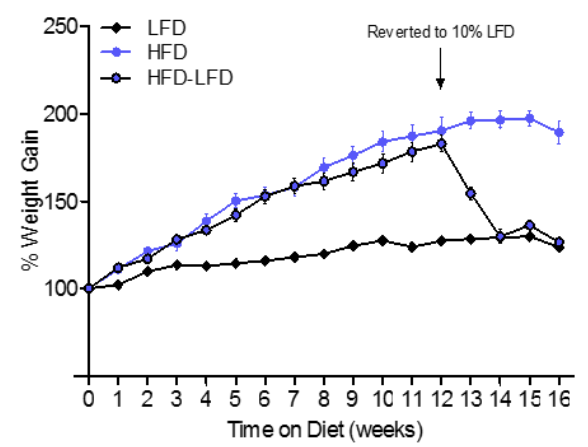

c.

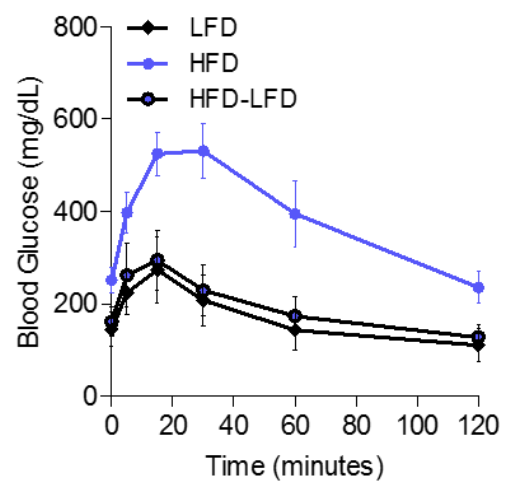

E.

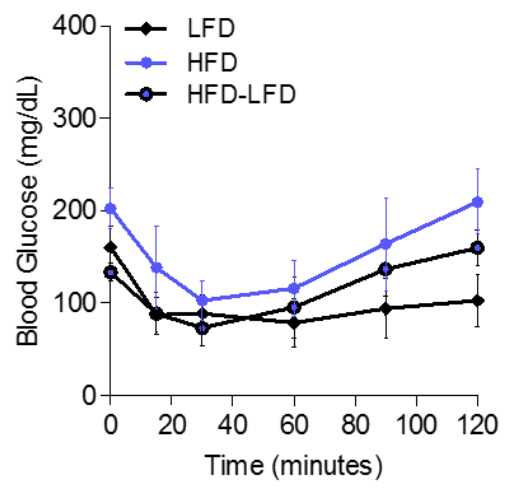

B.

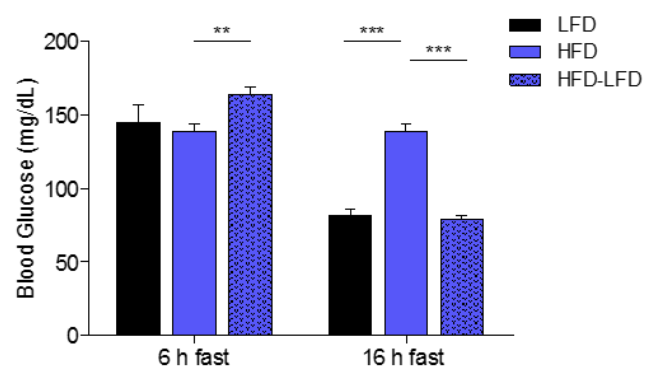

D.

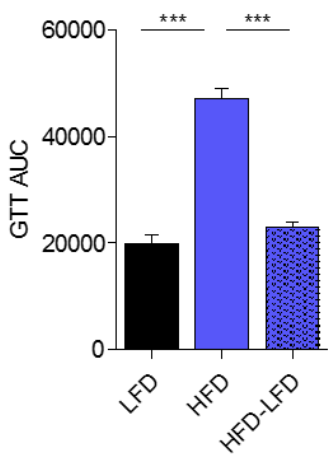

F.

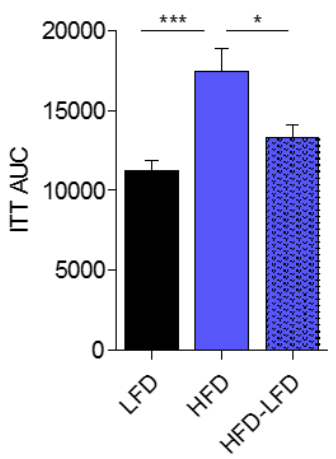


Figure 19. Changes in adiposity, glucose tolerance, and insulin tolerance after reversion to $10 \%$ LFD. Weekly weight gain was quantified and presented as percentage of the initial body weight, (A). Blood glucose levels after 6 and 16 hour fast can be found in graph (B). Changes in blood glucose level during a glucose tolerance test is presented in $\mathrm{mg} / \mathrm{dL},(C)$, and the area under the curve is quantified to the right, (D). Insulin induced changes in blood glucose level are plotted in (E) and quantification of area under the curve is presented in graph (F); $\mathrm{n}=8-18$ per group; ${ }^{*} p \leq 0.05,{ }^{* *} p \leq 0.01$, and ${ }^{* * *} p \leq 0.001$. 
Figure 20

A.

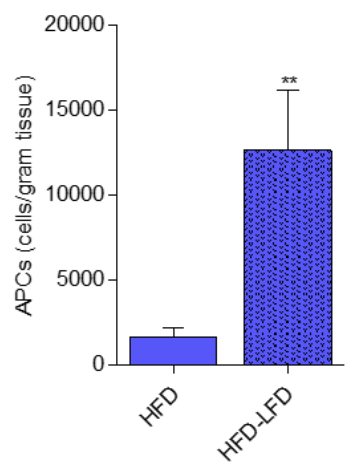

B.

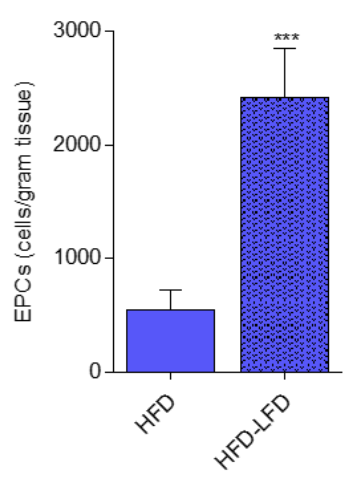

C.

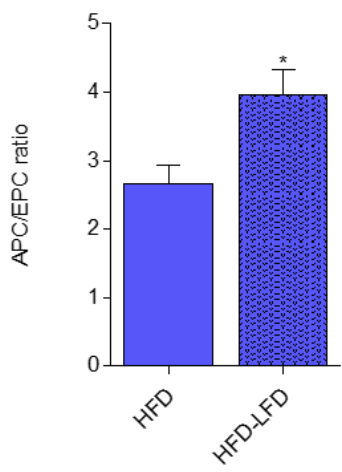


Figure 20. Quantification of adipocyte progenitor cell, APC, and endothelial progenitor cell, EPC, populations of visceral adipose tissue after consumption of $60 \%$ high fat diet for 12 weeks and reversion to $10 \%$ LFD for 4 weeks. The quantification for the total number of CD29+ APCs per gram of visceral adipose tissue are presented above in graph $(A)$. Quantification of the total number of Sca-1+ Flk-1+ EPCs per gram of visceral adipose tissue is also included in graph (B). The ratio of APCs to EPCs is presented in graph (C); $n=9$ 10 per group, ${ }^{*} p \leq 0.05,{ }^{* *} p \leq 0.01$, and ${ }^{* * *} p \leq 0.001$ vs. HFD. 


\section{Discussion}

The major findings of this study reveal that progenitor cell balance may be essential to promote protective adipose tissue remodeling and that adipocyte progenitors are recruited from the bone marrow under conditions of nutrient excess. Our data show that 12 weeks of $60 \%$ HFD is sufficient to induce an obese phenotype in wild-type mice. Mice fed HFD exhibited impaired glucose tolerance, decreased physical activity, and increased body fat mass, primarily due to expansion of the visceral adipose tissue depot. This increase in adiposity increased the ratio of APCs to EPCs by 5-fold. Lineage tracing experiments confirmed that putative APCs are derived from bone marrow and that Sca-1+ Flk1+ EPCs are primarily resident to the tissue. While it has been shown that APCs can differentiate into mature adipocytes in vivo, the significance of the proportion of adipogenic and angiogenic precursors necessary to promote protective adipose tissue remodeling has not be fully described $(37,66)$.

Our data show a 2.9-fold increase in macrophages and 1.9-fold increase in T cells after 12 weeks of HFD, which is in accordance with numerous studies showing that obesity promotes a chronic state of inflammation $(55-57,167,174)$. Adipose tissue hypoxia has also been described to occur with obesity and is implicated as a potential contributor to the development of insulin resistance (55$59,172)$. EPCs have the capacity to repair damaged endothelium and to create new blood vessels in vivo $(68,69,71,79-82)$. We did not see a significant change in EPC number after 12 weeks of HFD, but the increase in APC to EPC ratio could be indicative of a diet-induced change in the stem cell composition of 
adipose tissue; one which might impact adipose tissue remodeling. It is important to note, more than $96 \%$ of Sca- $1+$ Flk1 + cells were GFP- in mice consuming HFD, suggesting bone marrow-derived EPCs are not likely recruited to the adipose tissue during obesity. Moreover, we find that HFD appears to decrease the proliferation of tissue-resident cells, suggesting that, these resident EPCs might have decreased proliferative capacity and contribute to pathological adipose tissue remodeling. This is important because decreased levels of these pro-angiogenic cells have been implicated in the development of several chronic diseases including cardiovascular disease, due likely to a diminished capacity to repair damaged vasculature (73-78). Obesity along with exposure to other environmental factors, i.e. air pollution and cigarette smoke, have been shown to negatively affect EPC numbers and function $(68,69,71,72)$.

The imbalance of APCs to EPCs is an interesting finding of this study. Protective adipose tissue remodeling is characterized by the presence of large numbers of small adipocytes, decreased adipose tissue inflammation, and maintenance of metabolic homeostasis $(56,62,63)$. APCs have been shown to proliferate, differentiate, and take up esterified lipid, expanding the adipose tissue (37). Our studies show that HFD increases these cells in visceral adipose tissue and that this increase may be due to increased recruitment from the bone marrow since $>96 \%$ of APCs were GFP+. The tissue-resident origin of the unchanged Sca-1+Flk1+ EPC population in visceral adipose tissue could provide insight into the preference for pathological remodeling under conditions 
of nutrient excess. Correcting the imbalance of these two cell types could be important in the discovery of novel therapeutic treatments for obesity.

Addressing the question of whether the ratio of APCs to EPCs is crucial in promoting protective adipose tissue remodeling in conditions of nutrient excess would require interventions to correct the imbalance of these two cell populations in adipose tissue. Increasing the EPC population by way of cell transplantation or stimulation of EPCs to mobilize from the bone marrow to visceral adipose tissue with HFD are two methods by which this could be addressed. However, we have observed that subjecting mice to repeated injections can prevent body weight gain (unpublished observations). Because the goal of these studies would be to examine the adipose tissue remodeling process, the decreased weight gain could skew results. To mobilize the bone marrow EPCs pharmacologically, it would be advantageous to select an agent with minimal interactions with other progenitor cell types so that the contribution of mobilized cells to the remodeling processes could be clearly discerned.

In summary, our work provides information about the origins of adipose tissue stem cells and the diet-induced changes in their relative abundance. We find that 12 weeks of HFD substantially increases gross tissue mass and inflammation, which was associated with increases in the ratio of APCs to EPCs. We also find that APCs appear to derive from the bone marrow and that Flk+ Sca+ EPCs are primarily tissue-resident. The observation that tissue-resident stromal cells had lower BrdU positivity suggests that this population of EPCs could have defective proliferative capacity during conditions of nutrient excess. 
Overall, these findings support our hypothesis that obesity impairs adipose tissue remodeling by negatively affecting stem cells and lends insight into a possible mechanism, i.e., that obesity impairs proliferation of adipose tissue-resident EPCs, leading to a decreased capacity to provide vascular support to forming, newly formed, or mature adipocytes. 


\section{CHAPTER 4 \\ CONTRIBUTION OF STEM CELLS TO ADIPOSE TISSUE REMODELING}

\section{Introduction}

Stem cells play an important role in tissue homeostasis and repair $(16,56$, $63,70,71,175)$. Mesenchymal stem cells (MSCs) have been successfully isolated from multiple tissues including skeletal muscle, adipose tissue, and bone marrow $(37,66,176-178)$. These cells have also been shown to be give rise to several cell types including skeletal muscle, heart, joint, bone, and adipose tissue (177, 179-181). MSCs isolated from skeletal muscle are relatively easy to obtain via muscle biopsy. Satellite cells, progenitor cells which are known to give rise to myoblasts, are located in close proximity to myofibers and though many of these cells are committed to the myogenic lineage, these CD29 positive cells can also undergo chondrogenic, endothelial, and osteogenic differentiation (177, 180, 181). Bone marrow-derived cells expressing early cardiac markers as well as Sca-1, CD34 and AC133 have been shown to migrate to the damaged myocardium post myocardial infarction and are believed to be involved in repair of myocardium (179). In adipose tissue, there is a vast pool of progenitor cells including adipocyte progenitors (APCs), which can differentiate into mature adipocytes, and endothelial progenitors (EPCs), which promote the formation of new blood vessels as well as provide support for existing vasculature. There is also evidence that these adipose tissue stem cells can impose paracrine effects, 
stimulating the migration of endothelial cells to prevent neointimal formation and promote vascular repair $(37,52,66,169,182,183)$. Since stem cells play a role in several organ systems, the competency and levels of these cells could be crucial to their ability to provide adequate tissue support; however, both of these characteristics can be affected by several factors $(52,68,69,73,75,82,168)$.

In adipose tissue, increased levels of stem cells could be indicative of favorable adipose tissue remodeling. Increasing these cell populations may allow for massive expansion of adipose tissue in response to HFD, while maintaining metabolic homeostasis. This results in the development of a benign state of morbid obesity predicated by an increased number of small adipocytes and systemic insulin sensitivity. For example, increased levels of adiponectin, a hormone secreted by adipose tissue, stimulated adipocyte progenitors to differentiate. In so doing, lipid uptake and energy expenditure of white adipose tissue are increased $(62,63)$.

In the obese condition, adipocytes can expand tremendously to accommodate the excess lipid load, and in some cases, adipocytes hypertrophy to $10,000 \mu \mathrm{m}$ in diameter (184). Since oxygen has a limited diffusion capacity in which it can only supply cells within a close proximity to a functional capillary bed, such an increase in adipocyte area creates a hypoxic environment in obese adipose tissue $(58,185-187)$. Hypoxic conditions have been shown to inhibit adipogenic differentiation of mesenchymal stem cells (MSCs) from bone marrow and adipose tissue (188-191). Hypoxia also has an effect on the endocrine function of adipose tissue, suppressing production of adiponectin by adipocytes 
$(185,186,192,193)$. Adiponectin is a hormone which has been shown to support proliferation of adipocytes as well as decrease inflammation in adipose tissue and vasculature (194). Adipose tissue of obese, transgenic mice that overexpress adiponectin, have been shown to have an increased number of smaller adipocytes when compared to obese controls (63). Adiponectin also acts on endothelial cells to increase the production of nitric oxide (194), which is significantly decreased with obesity (109). Nitric oxide (NO) plays a role in a host of physiological processes including the regulation of blood flow, angiogenesis, and vasodilatation. In addition, NO and other reactive oxygen species have been shown to increase differentiation of preadipocytes or APCs in vitro (195-197). When preadipocytes were treated with an NO releasing agent, hydroxylamine, or a substrate of nitric oxide synthase, L-arginine, lipoprotein lipase and glycerol-3phosphate dehydrogenase were increased indicating adipogenic differentiation. Inducible nitric oxide synthase (iNOS) and endothelial nitric oxide synthase (eNOS) were both upregulated in differentiating cells, however differentiation was uninterrupted upon inhibition of iNOS suggesting that eNOS may have a more significant role in adipogenic differentiation of preadipocytes (197).

Data from our previous study show no significant change in the number of adipocyte progenitor and endothelial progenitor cells after 12 weeks of high fat feeding. However, the ratio of the two cell populations is significantly increased with HFD. As previously described, both endothelial and adipocyte progenitor cells are necessary for the positive remodeling of the adipose tissue. Since obesity disrupts the balance of these two progenitor populations, we chose to 
examine if correcting this imbalance might support a more metabolically favorable adipose tissue remodeling, improving diet-induced glucose intolerance and decreasing adiposity. To do so, we increased the EPC population in adipose tissue of mice fed HFD by cell transplantation in an attempt to correct the APC to EPC ratio associated with HFD. As an additional way of potentially improving vascular function, we also sought to determine if increasing expression of eNOS in bone marrow-derived cells would support favorable adipose tissue remodeling by increasing APC differentiation or increasing vascular perfusion.

To supplement EPCs in adipose tissue of obese mice, we performed a direct cell transplantation of bone marrow-derived EPCs isolated from wild-type or eNOS-Tg mice into visceral adipose tissue. After 12 weeks of $60 \%$ HFD, mice transplanted with eNOS-Tg bone marrow showed no difference in adipocyte size, glucose, or insulin tolerance when compared to WT transplanted group.

However, there was an appreciable increase in adipose tissue vascularity of high fat-fed mice transplanted with eNOS-Tg bone marrow. Results from this study suggest that increasing the competency or number of progenitor cells may support angiogenesis in the obese condition.

\section{Experimental procedures}

Animal Studies and Procedures: All procedures were approved by the University of Louisville Institutional Animal Care and Use Committee. Four week old B6.SJL-Ptprca Pepcb/BoyJ (Pep Boy; wild-type; WT) mice were purchased from The Jackson Laboratory (Bar Harbor, ME). The mice were given several days to 
acclimate to the animal facility before being subjected to a lethal dose of $\gamma$ radiation (950cGy) by way of a cesium source (173). Twenty-four hours after irradiation, bone marrow from 8 week old male eNOS-Tg and WT mice was isolated and used to transplant irradiated WT mice via a single retro-orbital injection. Approximately, $1 \times 10^{7}$ bone marrow stromal cells were injected into each mouse using $100 \mu \mathrm{l}$ sterile PBS as the vehicle. The WT mice were purchased from The Jackson Laboratory (Bar Harbor, ME) and the colony of eNOS-Tg mice was maintained at the University of Louisville. The transgenic mice were developed on a C57BL/6J background and the animals overexpress bovine eNOS due to manipulation of the preproendothelin-1 promoter. Five weeks after transplantation, at 9 weeks of age, bone marrow transplanted (BMT) mice were placed on either a 10\% low fat diet (LFD; Research Diets, Inc., \#D12450B) or a 60\% high fat diet (HFD; Research Diets Inc., \#D12492) for 12 weeks. Water and diet were provided ad libitum and body weights were recorded each week.

Cell isolation: Bone marrow stromal cells were isolated from the tibia and femur of both hind limbs of 8 week old GFP positive mice (The Jackson Laboratory, \#006567). The cells were flushed from the bones using a sterile solution of PBS with 10\% FBS and a 24G-needle and syringe and kept on ice until all cells had been isolated. Once the cells were isolated, the cell solution was underlayed with Fico/Lite-LymphoH (Atlanta Biologicals, 140150) in a 1:1 fashion in sterile conical tubes. The tubes were then centrifuged for 20 minutes at $1500 \mathrm{G}$ at $18^{\circ} \mathrm{C}$. The 
interphase layer, or buffy coat, was carefully removed and the cells were washed twice.

The stromal vascular fraction (SVF) was isolated from the visceral and subcutaneous adipose depots. The adipose tissue was finely minced and digested in collagenase (500CDU/mL PBS/HEPES) at $37^{\circ} \mathrm{C}$ for 25 minutes. The mixture was then filtered to remove any particles larger than $100 \mu \mathrm{m}$ and washed several times to isolate the SVF cells for analysis.

Cell culture: The isolated cells were cultured on Fibronectin-coated (SigmaAldrich, F0895) 6 well dishes with $2 \mathrm{~mL}$ of culture medium. The culture medium consists of EBM-2 basal medium (Lonza, CC-3156), EGM-2 SingleQuot Kit supplements and growth factors (Lonza, CC-4176), FBS (Atlanta biologicals, S11150H) and Penicillin-Streptomycin solution (Gibco, 15070-063). 48 hours after seeding the cells, the medium was refreshed. Medium was then changed every 24 hours for the next 3 days, then every 2 days until the cells were confluent.

EPCs were removed from confluent 6 well plates using trypsin and neutralized with trypsin neutralization solution. This process usually takes some time because the cells tend to adhere tightly to the culture dish. $500 \mu$ trypsin was placed in each well for 5 minutes and then neutralized with $1 \mathrm{~mL}$ of solution. This process was repeated 2 more times to remove the majority of the cells. The cells were the washed with PBS and centrifuged at $500 \mathrm{G}$ for 5 minutes to pellet the cells. 
Endothelial Progenitor Cell transplantation: An aliquot of EPCs was used to calculate a total cell count, then aliquots of $1 \times 10^{7}$ cells were then placed into sterile Eppendorff tubes. The cells were then combined with Matrigel to bring the final volume to $500 \mu$ l. The solution was injected into the epidiymal fat pads of obese mice at 6 injection sites, 3 per pad, using a 24G-needle and syringe. Mice were monitored for 10 days post cell transplantation.

Body composition measurement: Lean mass, fat mass, percent body fat, and total body mass were measured by Dual X-ray Absorptiometry (DEXAscan) paired with a mouse densitometer (PIXImus; Lunar, Madison, WI). For this measurement, the body weight of each mouse was taken prior to the scan and animals were anesthetized with isofluorane gas for the duration of the DEXAscan. Measurements for each parameter were recorded and images of each animal were collected.

Glucose and Insulin Tolerance Tests: After a 6 hour fast, a bolus of glucose $(1.0 \mathrm{mg} / \mathrm{g}$ in sterile saline) was injected i.p. and blood glucose measurements were taken over the course of 2 hours using blood collected from the tail vein. For insulin tolerance test, a bolus of insulin (1.5U/kg Humulin R in sterile saline) was injected i.p. into non-fasted mice and blood glucose was measured over a 2 hour period.

Adipocyte area measurement: Samples of adipose tissue were removed from the visceral and subcutaneous depots. The samples were fixed in $10 \%$ formalin, embedded in paraffin, sectioned, and stained with hematoxylin and eosin for 
histological assessment. Adipocyte number and area were quantified using the Nikon Elements-Basic Research software package.

Immunostaining of adipose tissue: Isolectin was used to assess capillary density in the adipose tissue sections as described in Sansbury, et al. Circ Res, 2012. Staining for transplanted cells, adipocytes, and cell nuclei were done using primary antibodies against the following proteins; GFP, perilipin, and dapi, respectively. Distinct fluorescent secondary antibodies were used to detect each of the primary antibodies. This was done in collaboration with the Pathology CORE of the Diabetes and Obesity Center at the University of Louisville.

Imaging of adipose tissue: Images of the hematoxylin and eosin stained sections were taken using a Zeiss microscope from SPOT Imaging Solutions. The Zeiss confocal microscope belonging to the Department of Molecular Cardiology at the University of Louisville was used to generate images of the fluorescent-stained adipose tissue sections. The Carl Zeiss ZEN 2012- blue edition was used to capture and analyze each of the confocal images.

Analysis of adipose tissue by flow cytometry: SVF cells were isolated as previously described in this chapter and used for flow cytometric analysis. The Fc receptor was blocked for 10 minutes at $4^{\circ} \mathrm{C}$ to prevent non-specific antibody binding. The SVF cells were then stained for 30 minutes at $4^{\circ} \mathrm{C}$ with antibodies to detect each of the following cell populations: adipocyte progenitor cells (Sca-1, CD24, CD29, CD90.2), endothelial progenitor cells (Sca-1, Flk1), and hematopoietic cells (Sca-1+CD34+). All antibodies were (purchased from eBioscience or Biolegend) conjugated to unique fluorochromes for detection in 2 
or more distinct panels. Samples were analyzed using the BD LSR II and FACSDiva Software. Experiment files were exported and further analyzed using the FlowJo analysis software.

The WT mice that we used as recipients for the bone marrow transplant were "Pep Boy" mice ordered from The Jackson Laboratory. We chose to use these mice as recipients because they express the cell surface antigen CD45.1 instead of CD45.2, unlike the C57BL6/J mice which are commonly used as our WT model. The homozygous eNOS-Tg mice express CD45.2 so by using these two strains, we were able to assess the efficacy of the eNOS-Tg bone marrow transplant by flow cytometric analysis using antibodies against CD45.1 and CD45.2 purchased from eBioscience.

Statistical Analyses: Statistical analyses performed using the Prism GraphPad software. For direct comparison of two group, Student's t test was used. For comparison of multiple groups, one-way and two-way analysis of variance tests (ANOVA) were used with Bonferroni post-testing. Data are expressed as mean \pm standard error of the mean unless otherwise noted. Significant differences between groups are marked on the graph with "*” denoting $p$ value $\leq 0.05$, “*”" denoting $p$ value $\leq 0.01$, and "***" denoting $p$ value $\leq 0.0001$. 


\section{Results}

Adipocyte area is unchanged 10 days after direct cell transplantation.

Hematoxylin and eosin staining of adipose tissue sections reveal an increase in interstitial cells in visceral adipose tissue of obese mice compared to the subcutaneous depot (Figure 21A). Further histological analysis would need to be completed to determine the identity of these cells, however their presence might be indicative of adipose tissue remodeling during which inflammatory cells, stem cells, and extracellular matrix components are all significantly increased $(56,57$, 175). Adipocyte area measurement shows no significant difference between vehicle-treated and EPC-transplanted animals in either the visceral (vehicle, $6022 \pm 330.6$; EPC, $6345 \pm 539.4$ ) or subcutaneous (vehicle, $7435 \pm 655.9$; EPC, $7402 \pm 723.2$ ) adipose tissue depots (Figure 21B,C).

Transplanted cells engraft into capillaries of the visceral and subcutaneous adipose tissue within 10 days. After GFP+ EPC transplant, adipose tissue sections were fixed and stained to determine if integration of transplanted bone marrow cells occurred. Cells which are co-stained with isolectin and GFP represent transplanted bone marrow-derived cells which have integrated into the existing vascular network or formed a new blood vessel. As seen in Figure 22, several GFP positive cells in the visceral and subcutaneous adipose tissue of the transplanted mice are also isolectin positive. 
Figure 21

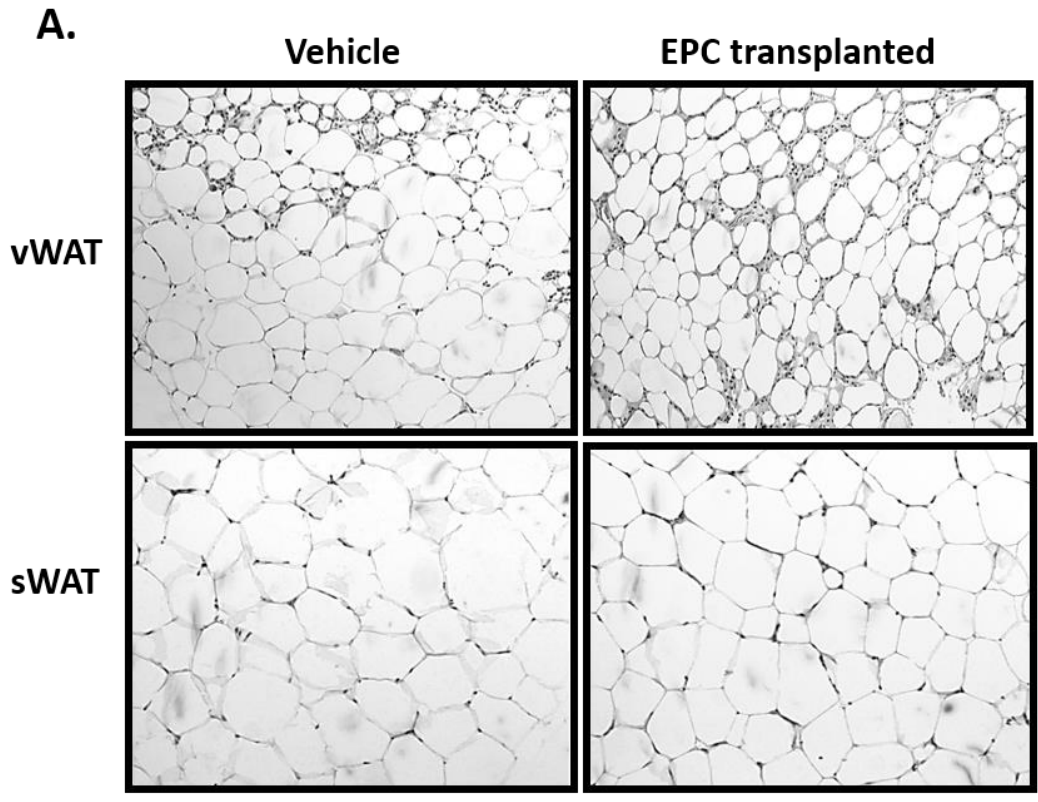

B.

c.
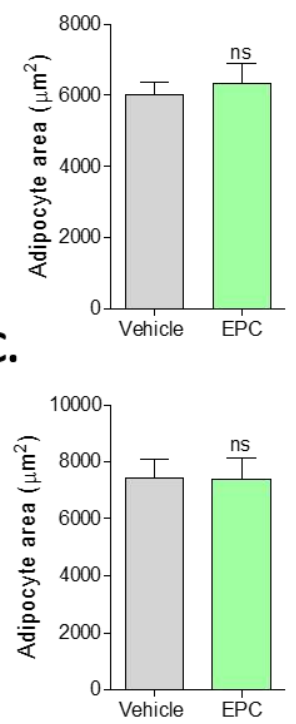
Figure 21. Hematoxylin and eosin stained paraffin-embedded visceral and subcutaneous adipose tissue sections. Visceral, vWAT, and subcutatneous, sWAT, adipose tissue sections from vehicle and EPC injected animals were stained for histological assessment, (A). These images were taken at $20 x$ magnification. Average adipocyte area of cells in the visceral adipose tissue and subcutaneous adipose tissue is quantified in graphs (B) and (C), respectively; $\mathrm{n}=3$ per group. 
Figure 22

A.

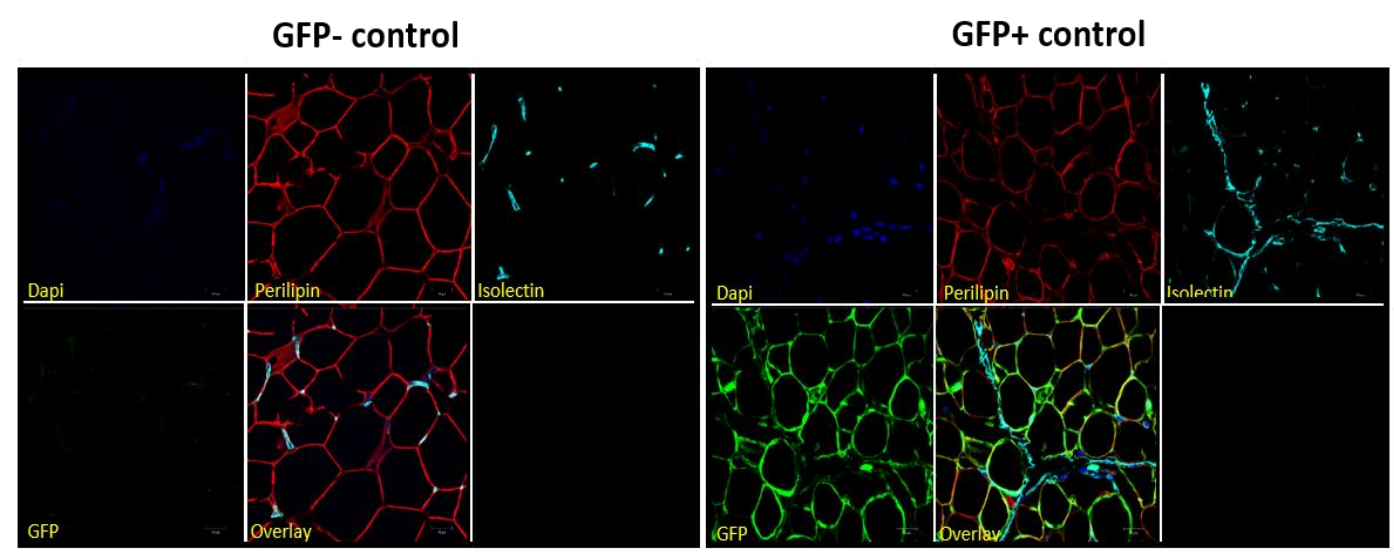

B.

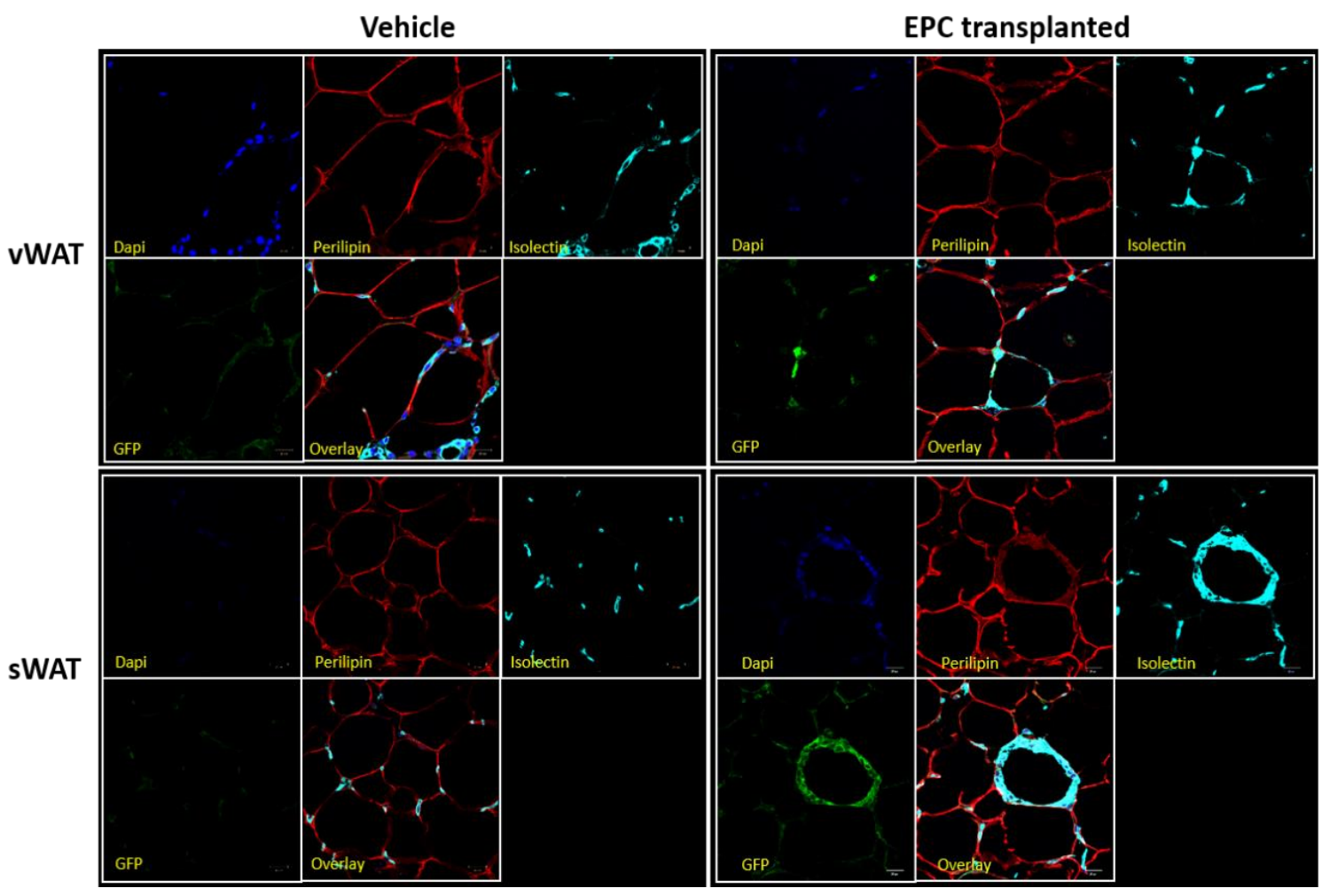


Figure 22. Representative confocal images of isolectin stained adipose tissue sections. Samples of visceral and subcutaneous adipose tissue were fixed in formalin, embedded in paraffin, sectioned, and stained to visualize GFP+ EPC integration into isolectin stained capillary networks. GFP negative and positive controls as well as representative images of subcutaneous and visceral adipose tissue from EPC-transplanted and vehicle-injected mice are presented, (A) and (B), respectively. 
Transplantation of eNOS-Tg stromal cells increased total body mass after 12 weeks of HFD. Total body weight and weekly weight gain were significantly increased with HFD in both mice transplanted with WT and eNOS-Tg bone marrow (Figure 23E-H). Dexascan analysis showed that total body mass was modestly increased (1.08-fold) in mice transplanted with eNOS-Tg bone marrow compared with mice transplanted with WT bone marrow after 12 weeks of HFD (Figure 23I). Percent body fat was increased 1.8-fold HFD in both the WT and eNOS-Tg transplanted groups (Figure 23J). Regardless of the diet, average lean mass was not different in mice transplanted with WT bone marrow; the lean mass of mice in the HF eNOS-Tg group was 1.1-fold higher than that of the LF eNOS-Tg group (Figure 23K). Fat mass increased 2.6-fold in eNOS-Tg transplanted animals and 2.4-fold in WT transplanted animals after 12 weeks of HFD (Figure 23L).

Systemic insulin sensitivity was not changed with transplantation of eNOS-Tg bone marrow. Fasting blood glucose levels were elevated 1.3-fold in eNOS-Tg HFD group compared to eNOS-Tg LFD after a $6 \mathrm{~h}$ fast (Figure 24A). After a $16 \mathrm{~h}$ fast, glucose levels were 1.7-fold higher in HF WT compared with LF WT and 1.4-fold higher in HF eNOS-Tg compared with LF eNOS-Tg (Figure 24A). Glucose and insulin tolerance tests showed a diet-dependent difference in area under the curve; however there was no significant difference between WT and eNOS-Tg transplanted groups (Figure 24D,G). 
Figure 23

A.

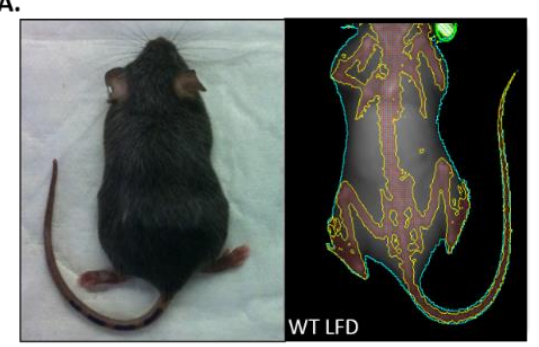

c.

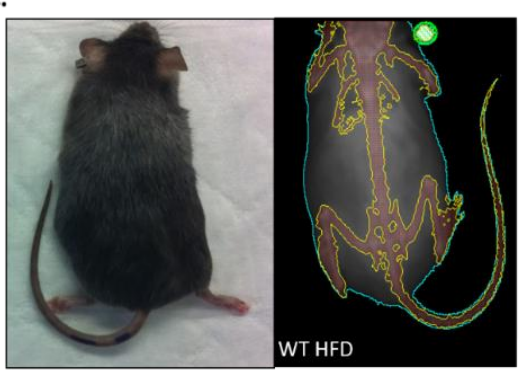

B.

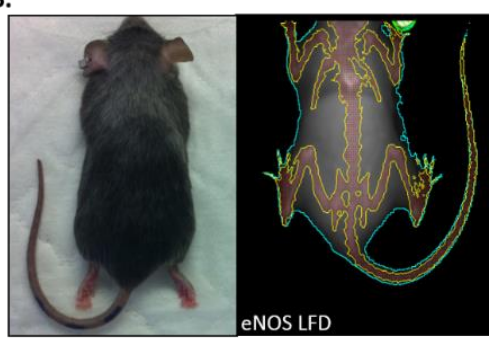

D.

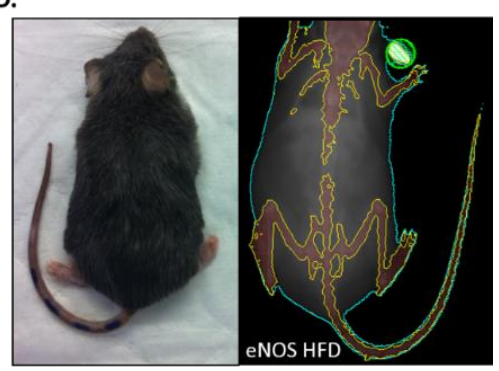

E.

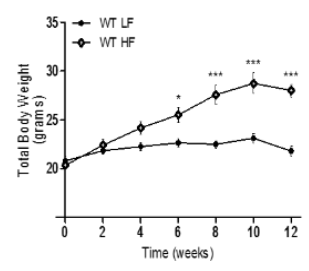

I.

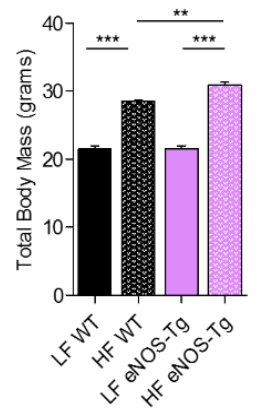

F.

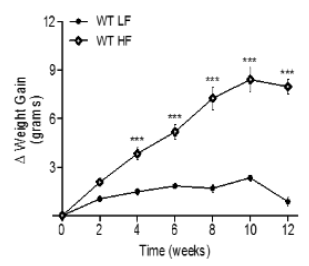

J.

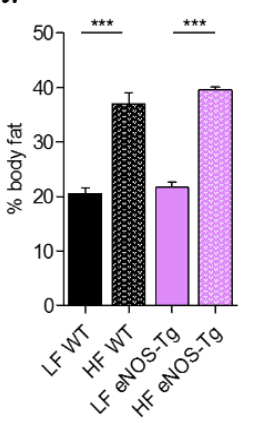

G.
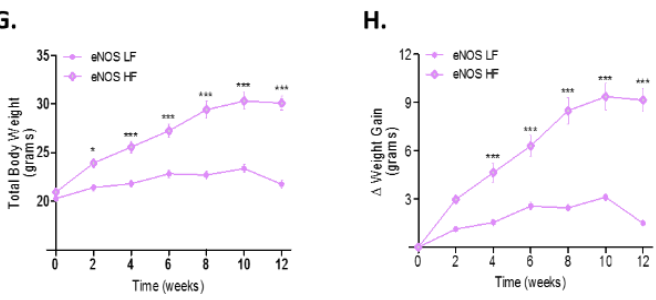

K.

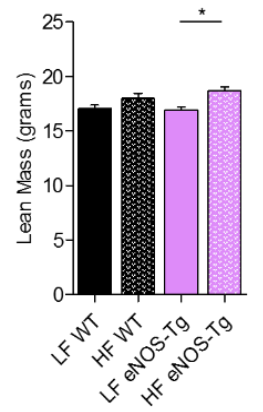

L.

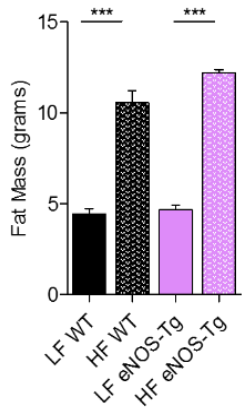


Figure 23. High fat diet increases adiposity in WT and eNOS-Tg bone marrow transplanted animals. Representative pictographs and Dexascan images of each group are presented and labeled accordingly, (A), (B), (C), and (D). Total body weight, $(E)$ and $(G)$, and delta weight gain, $(F)$ and $(H)$, are expressed in grams. Dexascan parameters, including total body mass (I), lean mass $(\mathrm{K})$, and fat mass $(\mathrm{L})$, are plotted in grams of tissue. Body fat expressed as a percentage of the total body mass is also included, graph $(\mathrm{J}) ; n=4$ per group; ${ }^{*} p \leq 0.05,{ }^{* *} p \leq 0.01$, and ${ }^{* * *} p \leq 0.001$. 
Figure 24

A.

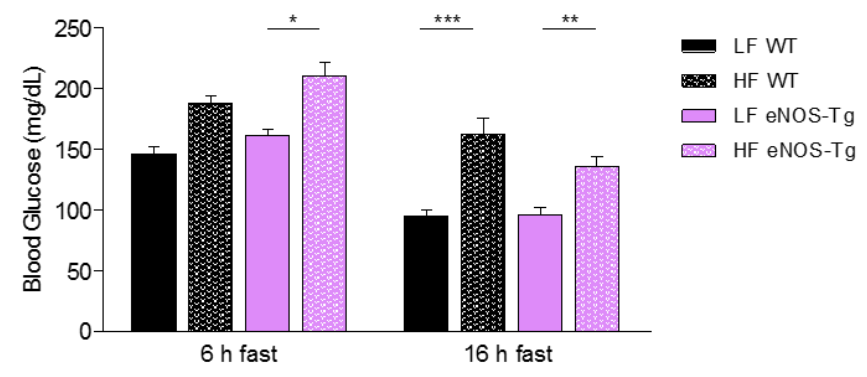

B.

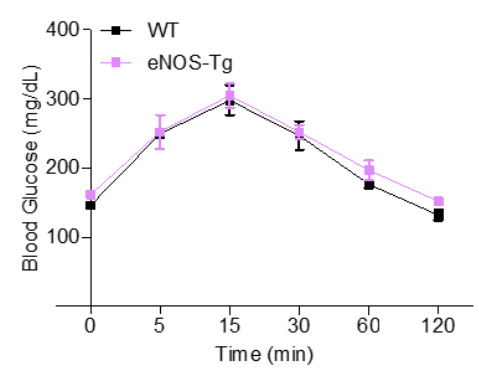

E.

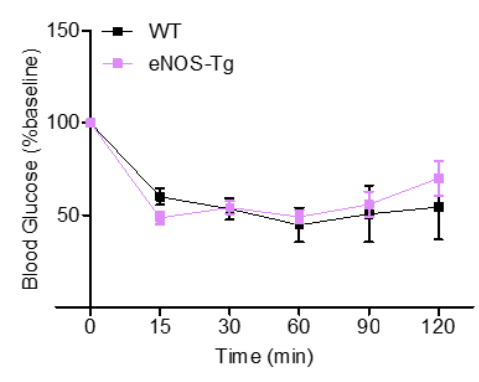

c.

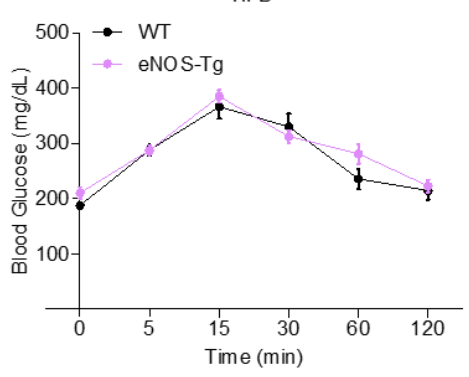

F.

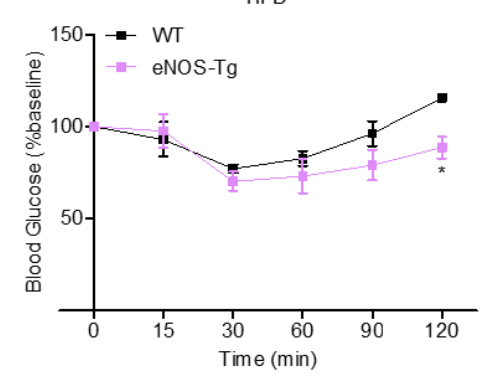

D.

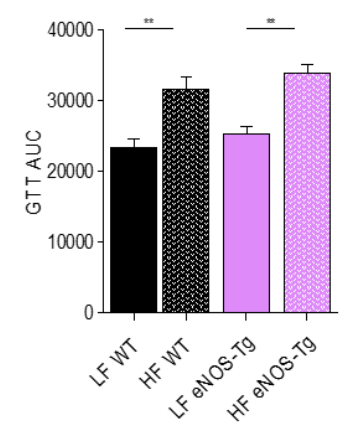

G.

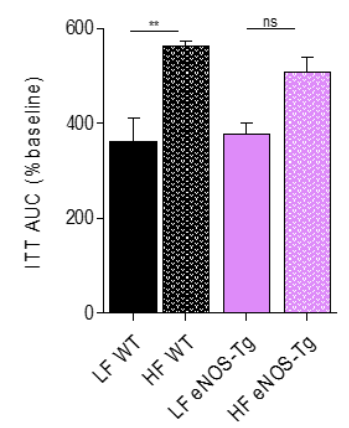


Figure 24. Measurements of insulin sensitivity in bone marrow transplanted animals after 12 weeks of high fat diet. After 12 weeks of a LFD or HFD, fasting blood glucose levels were measured after a 6 and 16 hour fast, $(A)$. A glucose tolerance test, (B) and (C), and insulin tolerance test, $(E)$ and $(F)$, was also administered. Area under the curve for the glucose tolerance test was calculated and expressed as such in graph (D). Area under the curve was calculated and normalized to the baseline glucose measurement for the insulin tolerance test, graph (G); $\mathrm{n}=4$ per group; ${ }^{*} p \leq 0.05,{ }^{* *} p \leq 0.01$, and ${ }^{* * *} p \leq 0.001$. 
High fat diet induces a progenitor cell imbalance in visceral adipose tissue. APCs were not significantly changed after 12 weeks of HFD (Figure 25A). EPCs were significantly decreased in all groups compared to LF WT (Figure 25B). APC to EPC ratio showed a significant increase in both high fat-fed groups compared to LF WT, 10.8-fold in HF eNOS-Tg and 10.2- fold in HF WT (Figure 25C). Since CD34 positive cells have been shown to promote the formation of vasculature in vivo $(79,169)$, changes in the CD34 positive cell population was also examined. Similar to the endothelial progenitor cell population, CD34 positive cells were decreased in high fat-fed groups, 3.4-fold in HF WT and 2.7-fold in HF eNOS-Tg (Figure 25D).

Isolectin staining is increased in adipose tissue of mice transplanted with eNOSTg bone marrow after 12 weeks of HFD. Isolectin staining in adipose tissue sections from LF-fed mice did not appear to be different between WT and eNOSTg transplanted groups (Figure 26A,B). In high fat-fed groups, isolectin staining appeared to be significantly increased in adipose tissue of mice that received eNOS-Tg bone marrow compared to WT (Figure 26C,D). In HF eNOS-Tg adipose tissue sections, formation of large vascular networks are visualized by positive isolectin staining which was not observed in any of the other groups. 
Figure 25

A.

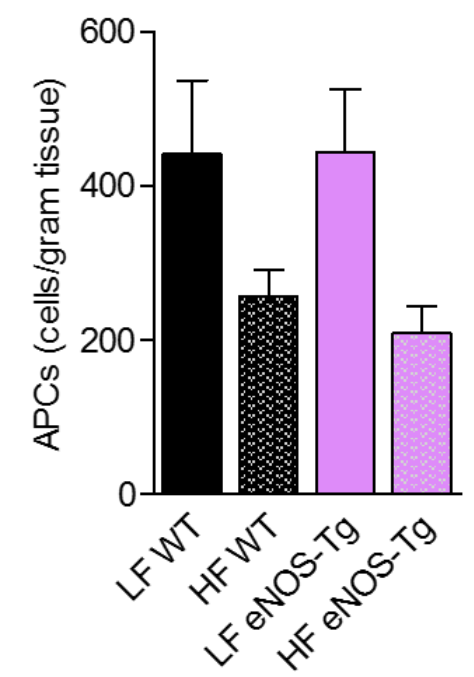

C.

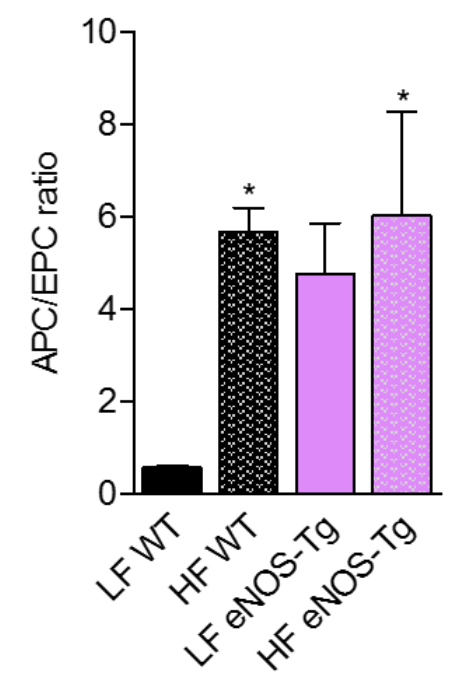

B.

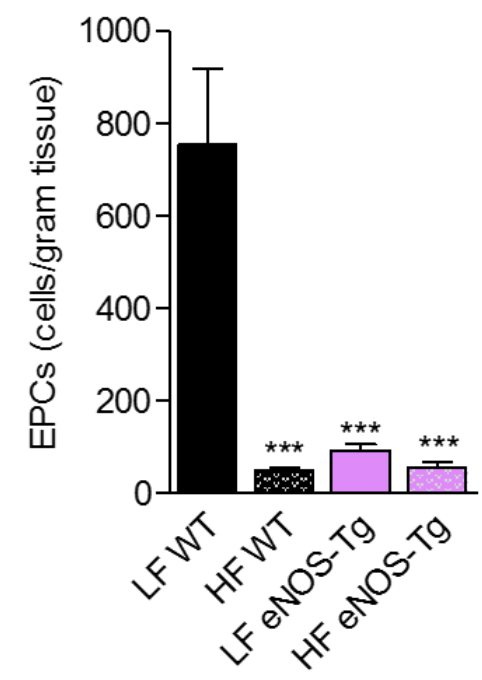

D.

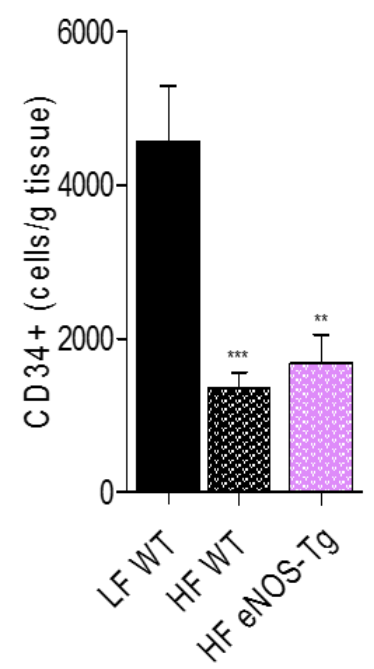


Figure 25. Quantification of progenitor cell populations of the visceral adipose tissue. Adipocyte progenitor cell, (A), and endothelial progenitor cell, (B), populations are quantified as cells per gram tissue. The ratio of these two cells types is presented in graph (C). CD34+ hematopoietic stem cells were also quantified as cells per gram tissue, (D); $n=9-10$ mice/ group, ${ }^{*} p \leq 0.05$ and ${ }^{* * *} p \leq 0.001$ vs. LF WT. 
Figure 26

A.

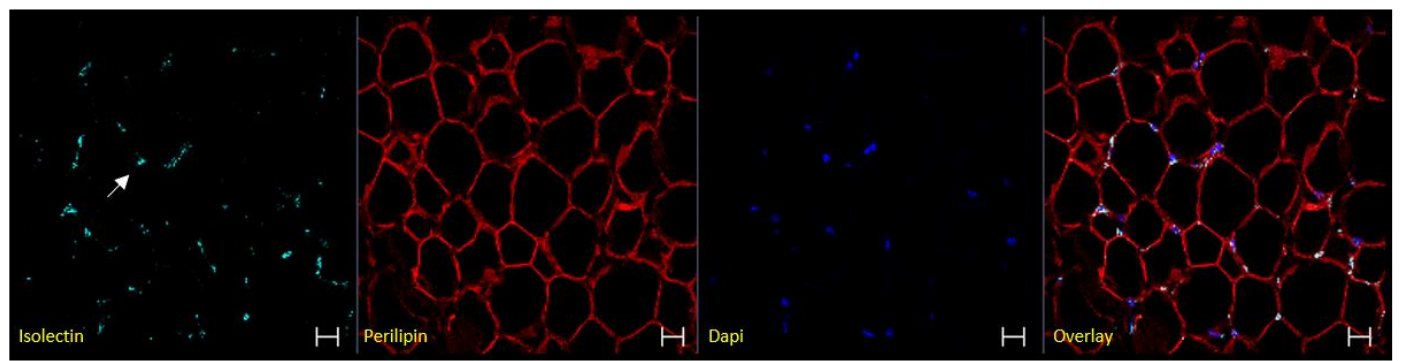

B.

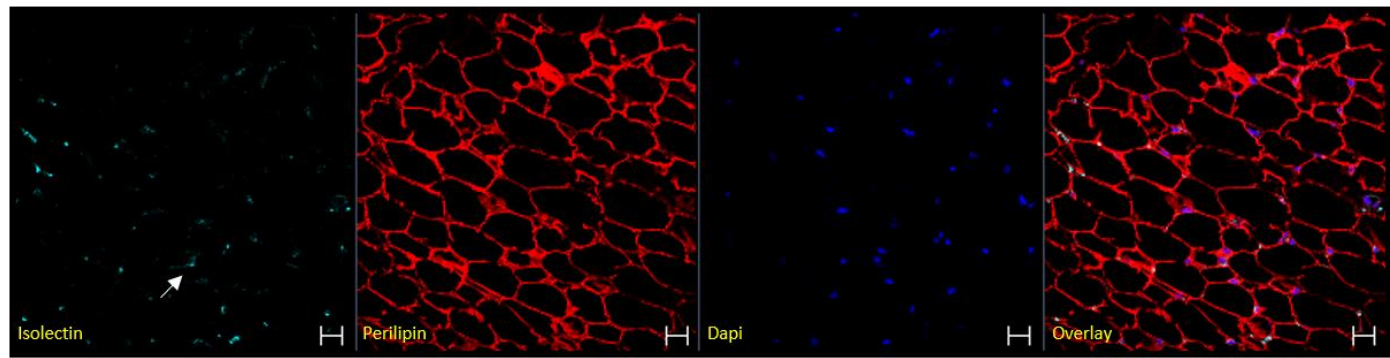

C.

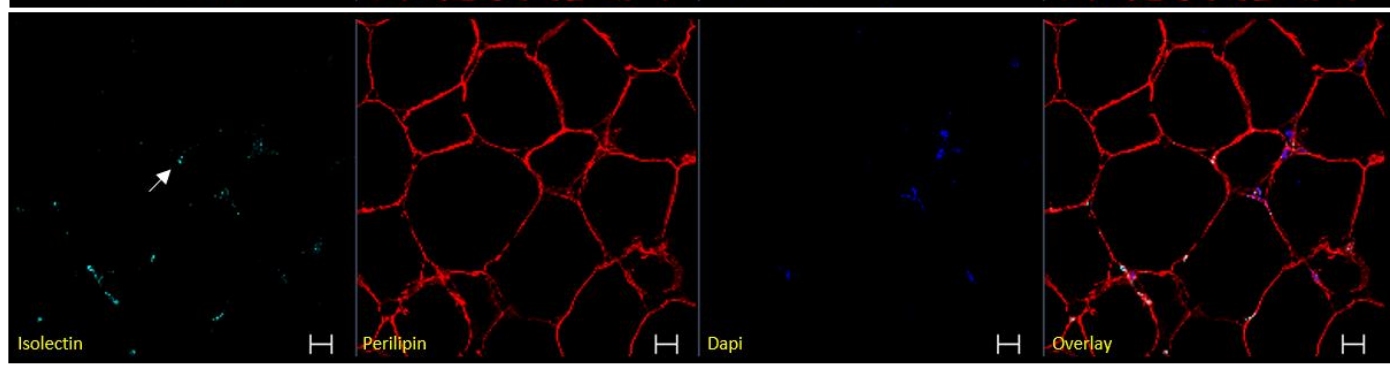

D.

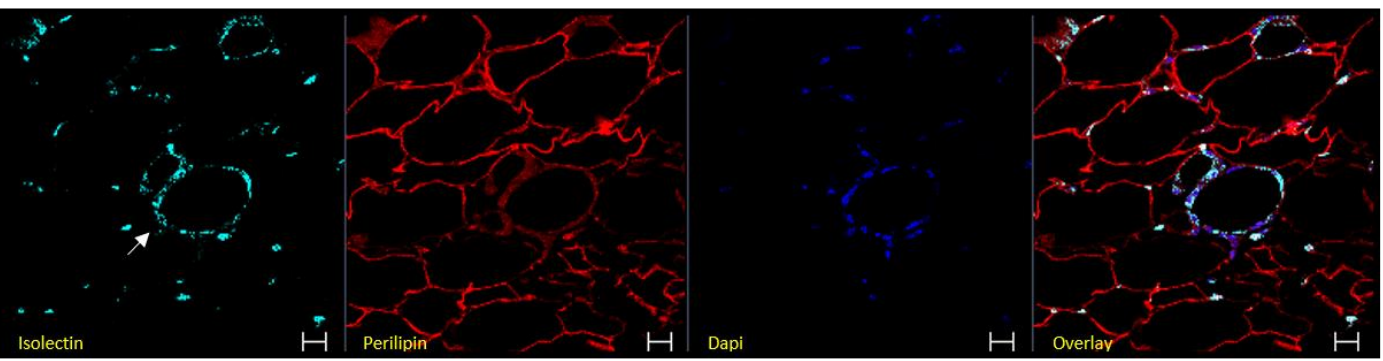


Figure 26. Isolectin staining of visceral adipose tissue sections after 12 weeks of LFD or HFD. Representative images of adipose tissue stained for isolectin, perilipin, and DAPI are presented in the figure; LF WT, LF eNOS-Tg, HF WT, and HF eNOS-Tg are represented by figures (A), (B), (C), and (D), respectively. White arrows mark isolectin positive cells. 


\section{Discussion}

Major findings of this study show that increased expression of eNOS by bone marrow cells promotes vascularization of adipose tissue under conditions of nutrient excess. We also observed an increase in vascularization of subcutaneous and visceral adipose tissue upon direct cell transplantation of bone marrow-derived EPCs. In association with insulin resistance and glucose intolerance occurring in mice fed HFD, we also observed a significant decrease in adipose tissue EPCs. Since it has been shown that vascular dysfunction and decreased nitric oxide may precede insulin resistance (109), we decided to perform a bone marrow transplant experiments to determine if increasing NO in bone marrow cells would promote favorable adipose tissue remodeling in conditions of nutrient excess. This study revealed a significant increase in APC to EPC ratio in all groups compared to WT LFD, due to a marked decline in Sca-1+ Flk1+ EPCs after 12 weeks of HFD and unchanged APC population. This supports our previous work suggesting that the imbalance of progenitor cell populations in the adipose tissue is diet dependent. It is also important to note that CD34 positive cells, which have also been identified as an endothelial progenitor populations $(75,79,169,170,198)$, were significantly decreased with HFD. Despite a marked increase in total body mass and a decreased number of EPCs, mice transplanted with eNOS-Tg bone marrow showed an apparent increase in vascularity of adipose tissue with HFD.

As previously discussed, NO has been shown to regulate differentiation of adipocyte progenitors $(79,197,199)$ and is significantly decreased with obesity 
(109). Repopulation of bone marrow with cells from mice overexpressing eNOS was intended to increase NO bioavailability and promote the formation of more vascularized capillary networks in adipose tissue. Our results show that vascularity of adipose tissue is significantly increased in HF-fed mice after transplantation of eNOS-Tg bone marrow cells, providing support for our hypothesis. This suggests that increasing eNOS may encourage the differentiation of progenitor cells in the adipose tissue to differentiate towards an endothelial lineage with HFD. If progenitors in the adipose tissue are in fact differentiating, this may account for the observed decrease we see upon analysis of the SVF.

Though there have been numerous studies examining the contribution of EPCs to angiogenesis $(75,79,169)$ and APCs to adipogenesis $(35,37,66)$, not much is has been reported about the significance of the ratio of adipocyte and endothelial progenitor cells in the context of adipose tissue remodeling. In this study, we examined how correcting the imbalance of these two cell populations in obese mice might impact adipose tissue remodeling. To counter the increase in bone marrow-derived APCs we observed in mice fed HFD, we increased the total EPC population in adipose tissue of obese mice by way of direct adipose tissue cell transplantation. The average adipocyte area was not changed, however engraftment of GFP positive donor cells into vasculature of subcutaneous and visceral adipose tissue was evident 10 days after injection of bone marrow-derived EPCs. Prior to injection, EPCs were cultured for several days under normoxic conditions. Exposure of bone marrow-derived stem cells to 
normoxic conditions has been shown to encourage differentiation (41). Perhaps, this "priming" of the transplanted bone marrow cells, increased their capacity to differentiate and incorporate into the vasculature in vivo.

Accurate identification of endothelial progenitor cells is a limitation which will hopefully be addressed by future studies in this field. Several different endothelial progenitor populations are currently being studied and collectively, these cells are referred to as "EPCs" because of the pro-angiogenic nature of the cell type $(68,69,71,78,80,168,170,200)$. In mice, Sca-1+ Flk1+ cells are considered to be EPCs $(69,71)$. However, this characterization may not include all pro-angiogenic progenitor populations. More precise identification of murine EPCs would be helpful in determining which specific progenitor populations are contributing to adipose tissue remodeling and may be beneficial in the discovery of interventions and therapies to treat obesity.

In summary, our work provides insight into the role that stem cells play in the vascularization of adipose tissue during obesity. Through our work we were able to show that an acute increase in bone marrow-derived progenitor cells in adipose tissue of obese mice results in the engraftment of donor cells into existing vasculature and new capillary networks. This could help correct the stem cell imbalance occurring in obesity or prime stem cells to differentiate. Our data also show that a long-term increase in eNOS, established by bone marrow transplantation, increases vascularity in adipose tissue of high fat-fed mice. Perhaps NO is important in the regulation of stem cells during conditions of nutrient excess. 


\section{CHAPTER 5 \\ CONCLUDING DISCUSSION}

The purpose of this dissertation was to understand better how adipose tissue remodels to accommodate excess nutrients during obesity. The most common cause for the development of obesity is hyperphagia resulting in the consumption of an increased amount of calories from fat (13-15). With this in mind, we used a nutrient excess model of obesity and examined structural, metabolic and compositional changes that occur in white adipose tissue during the development of obesity.

Evidence in the literature supports two forms of adipose tissue remodeling - a pathologic form, which appears to be the default during white adipose tissue expansion, and a protective form that allows for increased fat storage capacity and maintains systemic insulin sensitivity $(15,62,63)$. Adipocyte hypertrophy, insulin resistance, adipose tissue inflammation, and hypoxia are known to be associated with pathological expansion. Protective remodeling is characterized by proliferation of adipocyte progenitors in lieu of adipocyte hypertrophy. This type of remodeling allows for the preservation of metabolic homeostasis and insulin sensitivity; yet, why protective adipose tissue remodeling is not the default expansion method in diet-induced obesity remains unclear. The studies presented in this manuscript were completed in an effort to 
investigate the mechanisms by which nutrient excess affects the adipose organ.

\section{We hypothesize that obesity impairs stem cell-mediated adipose tissue remodeling.}

Prior to assessing this hypothesis directly, we first characterized the metabolic changes that occur in adipose tissue during obesity. This is important because metabolic changes and metabolites are now recognized as signaling mediators that regulate cell and tissue dynamics. To observe global metabolic changes after consumption of HFD, we completed several whole body physiological assessments as well as metabolomic analysis of adipose tissue. As described in Chapter 2, 6 weeks of HFD was sufficient to cause a marked increase in body fat composition as measured by Dexascan and an overall decline in physical activity was confirmed by metabolic cage analysis revealing decreased ambulatory and fine movements in mice consuming HFD. An increase in adipocyte area paired with substantial fat mass accumulation compared to LFD led us to investigate the metabolic changes in visceral adipose tissue after 6 and 12 weeks of HF-feeding.

Consumption of HFD has been shown to decrease mitochondrial abundance in adipose tissue (47-49). Our data showed that relative mitochondrial abundance was not different in mice fed HFD for 6 weeks compared to LF-fed group, however citrate synthase activity was decreased more than 2 fold. This enzyme is anchored to the mitochondrial matrix and is used in the Citric Acid Cycle for the conversion of acetyl-coA to citrate. Our 
results suggest that a decrease in citrate synthase activity may precede overt diminishment of mitochondria.

Insulin sensitizing drugs such as thiazolidinediones (TZDs) like pioglitazone and rosiglitazone have been shown to blunt this loss of mitochondria and even increase mitochondrial function in adipose tissue of obese individuals $(49,102)$. Interestingly, the role of TZDs has been examined in the context of stem cell biology. There is disagreement in the field about whether TZDs mediate the recruitment of bone marrow-derived cells to adipose tissue with consumption of HFD. Pioglitazone has been shown to decrease adipose tissue inflammation and adipose tissue macrophage accumulation induced by HFD (201). It was reported that rosiglitazone treatment encouraged engraftment of bone marrowderived cells in adipose tissue. This engraftment of bone marrow derived cells could also be seen to a lesser degree after feeding mice HFD. Data provided in this study suggest that the engrafted cells were multilocular in morphology and did not express CD11b or CD45, inflammatory cell markers (202). The next year, these results were countered suggesting that bone marrow-derived cells do not differentiate into adipocytes in vivo. Results of this study show that bone marrowderived cells accumulate in the interstitial space between adipocytes with HFD. These cells expressed CD11b, F4/80, LYVE-1 and CD45 indicating that these cells are most likely inflammatory cells (203). The major difference between these studies was the treatment with rosiglitazone. Perhaps TZDs not only impact mitochondrial abundance in mature adipocytes, but also promote adipogenic differentiation of bone marrow derived cells. 
Our studies revealed that high fat diet induces the recruitment of bone marrow-derived adipocyte progenitor cells to visceral adipose tissue after 12 weeks of HFD in the absence of pharmacological interventions. Characterization of these cells revealed that the bone marrow-derived population was not homogeneous. Characterization of SVF isolated from visceral adipose tissue showed more than $96 \%$ of the total APC population was derived from the bone marrow and approximately $20 \%$ of EPCs were bone marrow derived. However, upon analysis of intact adipose tissue, we did not observe any mature adipocytes which formed from the differentiation of a bone marrow-derived APC. We did however observe the engraftment of bone marrow-derived EPCs into vasculature of adipose tissue. Perhaps the metabolic changes in the adipose in response to HFD suppress the capacity of APCs to differentiate in conditions of nutrient excess.

One of the most robust changes that we observed via metabolomic analysis of visceral white adipose tissue of HF-fed mice was an increase in succinate. Succinate is an intermediate in the Citric Acid Cycle. The conversion of succinate to fumarate is catalyzed by Complex II, succinate dehydrogenase. This complex is composed of 4 subunits and after consumption of HFD for 12 weeks, the abundance of one component, succinate dehydrogenase B (SDHB), was significantly decreased. Succinate dehydrogenase has been shown to impact cell differentiation and proliferation. One such study showed mutations of the gene encoding for succinate dehydrogenase have been shown to be associated with normal citrate synthase levels, reduced oxygen consumption, 
and increase in HIF1- $\alpha$ which together increase cell migration and proliferation (204). HIF1- $\alpha$ has been shown to increase with obesity $(58,205)$. Succinate has also been shown to bind Gpr91, a Gi-coupled GPCR that regulates adipocyte function, and activate it (206). Upon binding its receptor in white adipose tissue, succinate inhibits lipolysis in adipocytes in vitro. (145). Perhaps the environment created by nutrient excess impacts the differentiation of bone marrow-derived APCs or the proliferation of tissue-resident EPCs.

In our assessment of the composition of the SVF isolated from HF-fed mice, we did not observe statistically significant changes in the number of APCs and EPCs. However, their relative abundance was changed, i.e., the ratio of APCs to EPCs was increased more than 5-fold with HFD. Since both endothelial and adipocyte progenitors are necessary for adipose tissue to expand in a metabolically favorable manner, the imbalance of these cell types could be critical for favorable adipose tissue remodeling.

As previously described, HFD is associated with a host of metabolic changes in adipose tissue which can lead to mitochondrial dysfunction. Since NO is a major regulator of mitochondrial biogenesis $(207,208)$ which is decreased with obesity, we experimented with increasing NO bioavailability under conditions of nutrient excess in the hope of generating a more perfused vascular network in the adipose tissue of obese mice. Interestingly, we found that HFD leads to a slightly greater increase in body mass of eNOS-Tg mice compared with WT transplanted mice. However, this did not induce changes in glucose tolerance, insulin tolerance, or stem cell abundance in adipose tissue compared to WT 
transplanted groups. Perhaps increasing NO via this route, i.e., via bone-marrow, is not advantageous to the adipose tissue remodeling process, or it is possible that conditions of HFD lead to eNOS uncoupling, which is in line with our previous findings (Sansbury Circ Res 2012). With regard to the former, this may be expected, as most of the EPCs in adipose tissue are resident to the tissue.

In chapter 4, we sought to determine the contribution of stem cells to adipose tissue remodeling. Since the ratio of APC to EPC was significantly increased with HFD, we chose to correct the imbalance of these cells via direct transplantation of EPCs into adipose tissue. This was specifically aimed to determine if supplementation of the deficient population could improve insulin and glucose handling. After just 10 days there was an observable increase in donor cell engraftment into capillary networks of the adipose tissue, indicating that the proportion of these progenitor cells may be important to the adipose remodeling process.

Engraftment of transplanted stem cells is a major issue in the field (209213), and culture conditions between cell isolation and transplant could modify cell competency. For example, exposure of bone marrow-derived stem cells to increased oxygen levels for 5 days has been shown to be sufficient to promote survival, growth, and cell migration when cells are reintroduced to hypoxia (214). Since bone marrow-derived EPCs were primed in a normoxic culture environment prior to injection, perhaps the cells were preconditioned to differentiate and contribute to vascular stability in vivo. 
In summary, these studies provide new and essential understanding for how conditions of obesity affect adipose tissue stem cells and adipose tissue remodeling. We find that diet-induced pathological adipose tissue remodeling is characterized by increased numbers of bone marrow-derived APCs and an increase in the APC to EPC ratio. We also find that APCs are derived predominantly from the bone marrow, whereas EPCs appear to be primarily resident to adipose tissue. Hence, metabolic changes occurring in the adipose organ during obesity could have a greater effect on tissue resident cells, such as EPCs. In line with this finding, BrdU staining in tissue-resident stromal cells of high fat-fed animals was lower than that of low fat-fed counterparts, which may be indicative of impaired EPC competency. Transplantation of EPCs directly into adipose tissue increased isolectin positivity, suggesting that they could increase vascular density, and this was augmented further when the EPCs overexpressed eNOS. Collectively, these studies demonstrate that obesity-induced derangement of adipose tissue stem cells in white adipose tissue is a modifiable target to combat obesity and its associated co-morbidities. Exciting prospects for future studies include addressing the potential metabolic underpinnings of obesity-related changes in adipose tissue stem cells and determining how restoration of stem cell competency affects obesity and its associated complications. 


\section{REFERENCES}

1. K. M. Flegal, M. D. Carroll, C. L. Ogden, C. L. Johnson, Prevalence and trends in obesity among US adults, 1999-2000. JAMA 288, 1723-1727 (2002).

2. R. J. Kuczmarski, K. M. Flegal, S. M. Campbell, C. L. Johnson, Increasing prevalence of overweight among US adults. The National Health and Nutrition Examination Surveys, 1960 to 1991. JAMA 272, 205-211 (1994).

3. C. L. Ogden, K. M. Flegal, M. D. Carroll, C. L. Johnson, Prevalence and trends in overweight among US children and adolescents, 1999-2000. JAMA 288, 1728-1732 (2002).

4. C. L. Ogden, M. D. Carroll, B. K. Kit, K. M. Flegal, Prevalence of obesity in the United States, 2009-2010. NCHS Data Brief, 1-8 (2012).

5. A. H. Mokdad et al., The spread of the obesity epidemic in the United States, 1991-1998. JAMA 282, 1519-1522 (1999).

6. (U.S. Department of Health and Human Services, Centers for Disease Control and Prevention, Altanta, GA).

7. C. L. Ogden, M. D. Carroll, B. K. Kit, K. M. Flegal, Prevalence of childhood and adult obesity in the United States, 2011-2012. JAMA 311, 806-814 (2014).

8. L. Zamosky, The obesity epidemic. While America swallows $\$ 147$ billion in obesity-related healthcare costs, physicians called on to confront the crisis. Medical economics 90, 14-17 (2013).

9. W. L. Haskell et al., Physical activity and public health: updated recommendation for adults from the American College of Sports Medicine and the American Heart Association. Circulation 116, 1081-1093 (2007).

10. P. T. Williams, Reductions in incident coronary heart disease risk above guideline physical activity levels in men. Atherosclerosis 209, 524-527 (2010).

11. R. C. Brownson, T. K. Boehmer, D. A. Luke, Declining rates of physical activity in the United States: what are the contributors? Annu Rev Public Health 26, 421-443 (2005).

12. N. Owen, P. B. Sparling, G. N. Healy, D. W. Dunstan, C. E. Matthews, Sedentary behavior: emerging evidence for a new health risk. Mayo Clin Proc 85, 1138-1141 (2010). 
13. C. Bouchard et al., The response to long-term overfeeding in identical twins. N Engl J Med 322, 1477-1482 (1990).

14. O. Ukkola, Y. A. Kesäniemi, A. Tremblay, C. Bouchard, Two variants in the resistin gene and the response to long-term overfeeding. Eur J Clin Nutr 58, 654-659 (2004).

15. T. D. Cummins et al., Metabolic remodeling of white adipose tissue in obesity. Am J Physiol Endocrinol Metab 307, E262-277 (2014).

16. G. H. Goossens, The role of adipose tissue dysfunction in the pathogenesis of obesity-related insulin resistance. Physiol Behav 94, 206218 (2008).

17. K. N. Frayn, C. M. Williams, P. Arner, Are increased plasma non-esterified fatty acid concentrations a risk marker for coronary heart disease and other chronic diseases? Clin Sci (Lond) 90, 243-253 (1996).

18. C. Bouchard, The biological predisposition to obesity: beyond the thrifty genotype scenario. Int J Obes (Lond) 31, 1337-1339 (2007).

19. C. Bouchard, Gene-environment interactions in the etiology of obesity: defining the fundamentals. Obesity (Silver Spring) 16 Suppl 3, S5-S10 (2008).

20. T. L. Nelson, G. P. Vogler, N. L. Pedersen, Y. Hong, T. P. Miles, Genetic and environmental influences on body fat distribution, fasting insulin levels and CVD: are the influences shared? Twin Res 3, 43-50 (2000).

21. E. S. Van Vleet et al., Neutral lipid components of eleven species of Caribbean sharks. Comp Biochem Physiol B 79, 549-554 (1984).

22. M. D. Jensen et al., 2013 AHA/ACC/TOS guideline for the management of overweight and obesity in adults: a report of the American College of Cardiology/American Heart Association Task Force on Practice Guidelines and The Obesity Society. Circulation 129, S102-138 (2014).

23. W. E. Siri, Body composition from fluid spaces and density: analysis of methods. 1961. Nutrition 9, 480-491; discussion 480, 492 (1993).

24. A. S. Jackson, M. L. Pollock, Generalized equations for predicting body density of men. Br J Nutr 40, 497-504 (1978).

25. A. S. Jackson, M. L. Pollock, A. Ward, Generalized equations for predicting body density of women. Med Sci Sports Exerc 12, 175-181 (1980).

26. J. Warolin et al., Comparative assessment of change in fat mass using dual X-ray absorptiometry and air-displacement plethysmography. Clin Obes 2, 66-72 (2012). 
27. S. Gesta, Y. H. Tseng, C. R. Kahn, Developmental origin of fat: tracking obesity to its source. Cell 131, 242-256 (2007).

28. R. M. McKay, J. P. McKay, L. Avery, J. M. Graff, C elegans: a model for exploring the genetics of fat storage. Dev Cell 4, 131-142 (2003).

29. B. Cannon, J. Nedergaard, Brown adipose tissue: function and physiological significance. Physiol Rev 84, 277-359 (2004).

30. B. B. Lowell et al., Development of obesity in transgenic mice after genetic ablation of brown adipose tissue. Nature 366, 740-742 (1993).

31. A. Bartelt, J. Heeren, Adipose tissue browning and metabolic health. Nat Rev Endocrinol 10, 24-36 (2014).

32. F. M. Fisher et al., FGF21 regulates PGC-1 $\alpha$ and browning of white adipose tissues in adaptive thermogenesis. Genes Dev 26, 271-281 (2012).

33. H. Ohno, K. Shinoda, B. M. Spiegelman, S. Kajimura, PPARy agonists induce a white-to-brown fat conversion through stabilization of PRDM16 protein. Cell Metab 15, 395-404 (2012).

34. J. E. Digby et al., Thiazolidinedione exposure increases the expression of uncoupling protein 1 in cultured human preadipocytes. Diabetes 47, 138141 (1998).

35. E. D. Rosen, O. A. MacDougald, Adipocyte differentiation from the inside out. Nat Rev Mol Cell Biol 7, 885-896 (2006).

36. P. A. Zuk et al., Multilineage cells from human adipose tissue: implications for cell-based therapies. Tissue Eng 7, 211-228 (2001).

37. M. S. Rodeheffer, K. Birsoy, J. M. Friedman, Identification of white adipocyte progenitor cells in vivo. Cell 135, 240-249 (2008).

38. Y. Feleke, D. Fekade, Y. Mezegebu, Prevalence of highly active antiretroviral therapy associated metabolic abnormalities and lipodystrophy in HIV infected patients. Ethiop Med J 50, 221-230 (2012).

39. A. K. Agarwal, A. Garg, Genetic disorders of adipose tissue development, differentiation, and death. Annu Rev Genomics Hum Genet 7, 175-199 (2006).

40. A. Garg, Clinical review\#: Lipodystrophies: genetic and acquired body fat disorders. J Clin Endocrinol Metab 96, 3313-3325 (2011).

41. P. Williams. (Arch Intern Med, 1997), vol. 157, pp. 191-198.

42. A. Guilherme, J. V. Virbasius, V. Puri, M. P. Czech, Adipocyte dysfunctions linking obesity to insulin resistance and type 2 diabetes. Nat Rev Mol Cell Biol 9, 367-377 (2008). 
43. O. Ilkun, S. Boudina, Cardiac Dysfunction and Oxidative Stress in the Metabolic Syndrome: an Update on Antioxidant Therapies. Current pharmaceutical design, (2013).

44. Y. Wang, Q. Yu, Y. Chen, F. Cao, Pathophysiology and therapeutics of cardiovascular disease in metabolic syndrome. Current pharmaceutical design, (2013).

45. H. Zhang, C. Zhang, Adipose "talks" to distant organs to regulate insulin sensitivity and vascular function. Obesity (Silver Spring) 18, 2071-2076 (2010).

46. F. Ippoliti, N. Canitano, R. Businaro, Stress and Obesity as Risk Factors in Cardiovascular Diseases: A Neuroimmune Perspective. Journal of neuroimmune pharmacology : the official journal of the Society on Neurolmmune Pharmacology, (2013).

47. H. J. Choo et al., Mitochondria are impaired in the adipocytes of type 2 diabetic mice. Diabetologia 49, 784-791 (2006).

48. L. N. Sutherland, L. C. Capozzi, N. J. Turchinsky, R. C. Bell, D. C. Wright, Time course of high-fat diet-induced reductions in adipose tissue mitochondrial proteins: potential mechanisms and the relationship to glucose intolerance. Am J Physiol Endocrinol Metab 295, E1076-1083 (2008).

49. I. Bogacka, H. Xie, G. A. Bray, S. R. Smith, Pioglitazone induces mitochondrial biogenesis in human subcutaneous adipose tissue in vivo. Diabetes 54, 1392-1399 (2005).

50. I. Bogacka, B. Ukropcova, M. McNeil, J. M. Gimble, S. R. Smith, Structural and functional consequences of mitochondrial biogenesis in human adipocytes in vitro. J Clin Endocrinol Metab 90, 6650-6656 (2005).

51. H. Chang, J. H. Park, K. H. Min, R. S. Lee, E. K. Kim, Whitening effects of adipose-derived stem cells: a preliminary in vivo study. Aesthetic Plast Surg 38, 230-233 (2014).

52. J. H. Kim, M. Jung, H. S. Kim, Y. M. Kim, E. H. Choi, Adipose-derived stem cells as a new therapeutic modality for ageing skin. Exp Dermatol 20, 383-387 (2011).

53. Q. A. Wang, P. E. Scherer, The AdipoChaser mouse: A model tracking adipogenesis in vivo. Adipocyte 3, 146-150 (2014).

54. B. E. Sansbury et al., Overexpression of Endothelial Nitric Oxide Synthase Prevents Diet-Induced Obesity and Regulates Adipocyte Phenotype. Circ Res, (2012).

55. S. Cinti et al., Adipocyte death defines macrophage localization and function in adipose tissue of obese mice and humans. J Lipid Res 46, 2347-2355 (2005). 
56. K. J. Strissel et al., Adipocyte death, adipose tissue remodeling, and obesity complications. Diabetes 56, 2910-2918 (2007).

57. K. Sun, C. M. Kusminski, P. E. Scherer, Adipose tissue remodeling and obesity. J Clin Invest 121, 2094-2101 (2011).

58. J. Ye, Emerging role of adipose tissue hypoxia in obesity and insulin resistance. Int J Obes (Lond) 33, 54-66 (2009).

59. I. S. Wood, F. P. de Heredia, B. Wang, P. Trayhurn, Cellular hypoxia and adipose tissue dysfunction in obesity. Proc Nutr Soc 68, 370-377 (2009).

60. C. M. Apovian et al., Adipose macrophage infiltration is associated with insulin resistance and vascular endothelial dysfunction in obese subjects. Arterioscler Thromb Vasc Biol 28, 1654-1659 (2008).

61. C. N. Lumeng, S. M. Deyoung, A. R. Saltiel, Macrophages block insulin action in adipocytes by altering expression of signaling and glucose transport proteins. Am J Physiol Endocrinol Metab 292, E166-174 (2007).

62. C. M. Kusminski et al., MitoNEET-driven alterations in adipocyte mitochondrial activity reveal a crucial adaptive process that preserves insulin sensitivity in obesity. Nat Med 18, 1539-1549 (2012).

63. J. Y. Kim et al., Obesity-associated improvements in metabolic profile through expansion of adipose tissue. J Clin Invest 117, 2621-2637 (2007).

64. I. W. Asterholm, P. E. Scherer, Enhanced metabolic flexibility associated with elevated adiponectin levels. Am J Pathol 176, 1364-1376 (2010).

65. E. D. Rosen, B. M. Spiegelman, Molecular regulation of adipogenesis. Annu Rev Cell Dev Biol 16, 145-171 (2000).

66. R. Berry, M. S. Rodeheffer, Characterization of the adipocyte cellular lineage in vivo. Nat Cell Biol 15, 302-308 (2013).

67. H. G. Linhart et al., C/EBPalpha is required for differentiation of white, but not brown, adipose tissue. Proc Natl Acad Sci U S A 98, 12532-12537 (2001).

68. P. Haberzettl et al., Exposure to ambient air fine particulate matter prevents VEGF-induced mobilization of endothelial progenitor cells from the bone marrow. Environ Health Perspect 120, 848-856 (2012).

69. T. E. O'Toole et al., Episodic exposure to fine particulate air pollution decreases circulating levels of endothelial progenitor cells. Circ Res 107, 200-203 (2010).

70. W. P. Cawthorn, E. L. Scheller, O. A. MacDougald, Adipose tissue stem cells meet preadipocyte commitment: going back to the future. $J$ Lipid Res 53, 227-246 (2012). 
71. L. A. Wheat et al., Acrolein inhalation prevents vascular endothelial growth factor-induced mobilization of Flk-1+/Sca-1+ cells in mice. Arterioscler Thromb Vasc Biol 31, 1598-1606 (2011).

72. M. Hristov, C. Weber, Progenitor cell trafficking in the vascular wall. $J$ Thromb Haemost 7 Suppl 1, 31-34 (2009).

73. M. Steinmetz, G. Nickenig, N. Werner, Endothelial-regenerating cells: an expanding universe. Hypertension 55, 593-599 (2010).

74. J. M. Hill et al., Circulating endothelial progenitor cells, vascular function, and cardiovascular risk. N Engl J Med 348, 593-600 (2003).

75. M. U. Becher, G. Nickenig, N. Werner, Regeneration of the vascular compartment. Herz 35, 342-351 (2010).

76. F. Shalaby et al., Failure of blood-island formation and vasculogenesis in Flk-1-deficient mice. Nature 376, 62-66 (1995).

77. N. Werner et al., Circulating endothelial progenitor cells and cardiovascular outcomes. N Engl J Med 353, 999-1007 (2005).

78. C. Schmidt-Lucke et al., Reduced number of circulating endothelial progenitor cells predicts future cardiovascular events: proof of concept for the clinical importance of endogenous vascular repair. Circulation 111, 2981-2987 (2005).

79. T. Asahara et al., Isolation of putative progenitor endothelial cells for angiogenesis. Science 275, 964-967 (1997).

80. R. M. Cubbon, M. B. Kahn, S. B. Wheatcroft, Effects of insulin resistance on endothelial progenitor cells and vascular repair. Clin Sci (Lond) 117, 173-190 (2009).

81. Q. Shi et al., Evidence for circulating bone marrow-derived endothelial cells. Blood 92, 362-367 (1998).

82. Q. Xiao et al., Sca-1+ progenitors derived from embryonic stem cells differentiate into endothelial cells capable of vascular repair after arterial injury. Arterioscler Thromb Vasc Biol 26, 2244-2251 (2006).

83. R. S. Ahima, Digging deeper into obesity. J Clin Invest 121, 2076-2079 (2011).

84. K. M. Flegal, M. D. Carroll, C. L. Ogden, L. R. Curtin, Prevalence and trends in obesity among US adults, 1999-2008. JAMA 303, 235-241 (2010).

85. Y. H. Tseng, A. M. Cypess, C. R. Kahn, Cellular bioenergetics as a target for obesity therapy. Nature reviews. Drug discovery 9, 465-482 (2010).

86. V. L. Roger et al., Heart Disease and Stroke Statistics--2012 Update: A Report From the American Heart Association. Circulation, (2011). 
87. D. W. Haslam, W. P. James, Obesity. Lancet 366, 1197-1209 (2005).

88. F. B. Hu, T. Y. Li, G. A. Colditz, W. C. Willett, J. E. Manson, Television watching and other sedentary behaviors in relation to risk of obesity and type 2 diabetes mellitus in women. JAMA 289, 1785-1791 (2003).

89. T. N. Robinson, Reducing children's television viewing to prevent obesity: a randomized controlled trial. JAMA 282, 1561-1567 (1999).

90. A. K. Kant, B. I. Graubard, Eating out in America, 1987-2000: trends and nutritional correlates. Prev Med 38, 243-249 (2004).

91. S. J. Nielsen, B. M. Popkin, Patterns and trends in food portion sizes, 1977-1998. JAMA 289, 450-453 (2003).

92. R. R. Briefel, C. L. Johnson, Secular trends in dietary intake in the United States. Annu Rev Nutr 24, 401-431 (2004).

93. Y. C. Wang, S. N. Bleich, S. L. Gortmaker, Increasing caloric contribution from sugar-sweetened beverages and $100 \%$ fruit juices among US children and adolescents, 1988-2004. Pediatrics 121, e1604-1614 (2008).

94. J. C. Wells, Thrift: a guide to thrifty genes, thrifty phenotypes and thrifty norms. Int J Obes (Lond) 33, 1331-1338 (2009).

95. L. M. Redman et al., Metabolic and behavioral compensations in response to caloric restriction: implications for the maintenance of weight loss. PLoS One 4, e4377 (2009).

96. R. L. Leibel, M. Rosenbaum, J. Hirsch, Changes in energy expenditure resulting from altered body weight. N Engl J Med 332, 621-628 (1995).

97. N. J. Rothwell, M. J. Stock, Luxuskonsumption, diet-induced thermogenesis and brown fat: the case in favour. Clinical science 64, 1923 (1983).

98. A. M. Cypess et al., Identification and importance of brown adipose tissue in adult humans. The New England journal of medicine 360, 1509-1517 (2009).

99. K. A. Virtanen et al., Functional brown adipose tissue in healthy adults. The New England journal of medicine 360, 1518-1525 (2009).

100. W. D. van Marken Lichtenbelt et al., Cold-activated brown adipose tissue in healthy men. The New England journal of medicine 360, 1500-1508 (2009).

101. A. Smorlesi, A. Frontini, A. Giordano, S. Cinti, The adipose organ: whitebrown adipocyte plasticity and metabolic inflammation. Obes Rev 13 Suppl 2, 83-96 (2012). 
102. L. Wilson-Fritch et al., Mitochondrial remodeling in adipose tissue associated with obesity and treatment with rosiglitazone. J Clin Invest 114, 1281-1289 (2004).

103. B. E. Sansbury et al., Overexpression of Endothelial Nitric Oxide Synthase Prevents Diet-Induced Obesity and Regulates Adipocyte Phenotype. Circulation Research 111, 1176-1189 (2012).

104. J. Hellmann, Y. Tang, M. Kosuri, A. Bhatnagar, M. Spite, Resolvin D1 decreases adipose tissue macrophage accumulation and improves insulin sensitivity in obese-diabetic mice. FASEB J 25, 2399-2407 (2011).

105. B. E. Sansbury et al., Metabolomic Analysis of Pressure-overloaded and Infarcted Mouse Hearts. Circulation. Heart failure, (2014).

106. J. Xia, D. S. Wishart, Web-based inference of biological patterns, functions and pathways from metabolomic data using MetaboAnalyst. Nature protocols 6, 743-760 (2011).

107. R. Horrillo et al., 5-lipoxygenase activating protein signals adipose tissue inflammation and lipid dysfunction in experimental obesity. J Immunol 184, 3978-3987 (2010).

108. J. A. Villena et al., Orphan nuclear receptor estrogen-related receptor alpha is essential for adaptive thermogenesis. Proc Natl Acad Sci U S A 104, 1418-1423 (2007).

109. F. Kim et al., Vascular inflammation, insulin resistance, and reduced nitric oxide production precede the onset of peripheral insulin resistance. Arterioscler Thromb Vasc Biol 28, 1982-1988 (2008).

110. G. S. Hotamisligil, P. Arner, J. F. Caro, R. L. Atkinson, B. M. Spiegelman, Increased adipose tissue expression of tumor necrosis factor-alpha in human obesity and insulin resistance. J Clin Invest 95, 2409-2415 (1995).

111. C. P. Sewter, J. E. Digby, F. Blows, J. Prins, S. O'Rahilly, Regulation of tumour necrosis factor-alpha release from human adipose tissue in vitro. $J$ Endocrinol 163, 33-38 (1999).

112. J. Xia, D. S. Wishart, MetPA: a web-based metabolomics tool for pathway analysis and visualization. Bioinformatics 26, 2342-2344 (2010).

113. T. Yamanouchi et al., Clinical usefulness of serum 1,5-anhydroglucitol in monitoring glycaemic control. Lancet 347, 1514-1518 (1996).

114. D. Stickle, J. Turk, A kinetic mass balance model for 1,5-anhydroglucitol: applications to monitoring of glycemic control. Am J Physiol 273, E821830 (1997).

115. B. G. Hill et al., Integration of cellular bioenergetics with mitochondrial quality control and autophagy. Biol Chem 393, 1485-1512 (2012). 
116. M. Spite et al., Deficiency of the leukotriene B4 receptor, BLT-1, protects against systemic insulin resistance in diet-induced obesity. Journal of immunology 187, 1942-1949 (2011).

117. S. Nishimura et al., CD8+ effector T cells contribute to macrophage recruitment and adipose tissue inflammation in obesity. Nat Med 15, 914920 (2009).

118. S. K. Chakrabarti, B. K. Cole, Y. Wen, S. R. Keller, J. L. Nadler, 12/15lipoxygenase products induce inflammation and impair insulin signaling in 3T3-L1 adipocytes. Obesity 17, 1657-1663 (2009).

119. S. K. Chakrabarti et al., Evidence for activation of inflammatory lipoxygenase pathways in visceral adipose tissue of obese Zucker rats. Am J Physiol Endocrinol Metab 300, E175-187 (2011).

120. C. S. Nunemaker et al., 12-Lipoxygenase-knockout mice are resistant to inflammatory effects of obesity induced by Western diet. Am J Physiol Endocrinol Metab 295, E1065-1075 (2008).

121. D. D. Sears et al., 12/15-lipoxygenase is required for the early onset of high fat diet-induced adipose tissue inflammation and insulin resistance in mice. PLoS One 4, e7250 (2009).

122. N. Yanaka, Mammalian glycerophosphodiester phosphodiesterases. Biosci Biotechnol Biochem 71, 1811-1818 (2007).

123. M. Prentki, S. R. Madiraju, Glycerolipid metabolism and signaling in health and disease. Endocr Rev 29, 647-676 (2008).

124. M. Gallazzini, M. B. Burg, What's new about osmotic regulation of glycerophosphocholine. Physiology 24, 245-249 (2009).

125. T. Bergsbaken, S. L. Fink, B. T. Cookson, Pyroptosis: host cell death and inflammation. Nature reviews. Microbiology 7, 99-109 (2009).

126. K. Schroder, J. Tschopp, The inflammasomes. Cell 140, 821-832 (2010).

127. A. Giordano et al., Obese adipocytes show ultrastructural features of stressed cells and die of pyroptosis. Journal of lipid research 54, 24232436 (2013).

128. B. L. Yu, S. P. Zhao, J. R. Hu, Cholesterol imbalance in adipocytes: a possible mechanism of adipocytes dysfunction in obesity. Obesity Reviews 11, 560-567 (2010).

129. S. Mitsutake et al., Dynamic modification of sphingomyelin in lipid microdomains controls development of obesity, fatty liver, and type 2 diabetes. J Biol Chem 286, 28544-28555 (2011).

130. Z. Li et al., Reducing plasma membrane sphingomyelin increases insulin sensitivity. Mol Cell Biol 31, 4205-4218 (2011). 
131. J. A. Chavez, S. A. Summers, A ceramide-centric view of insulin resistance. Cell Metab 15, 585-594 (2012).

132. C. Shah et al., Protection from high fat diet-induced increase in ceramide in mice lacking plasminogen activator inhibitor 1. J Biol Chem 283, 1353813548 (2008).

133. C. Schmitz-Peiffer, Targeting ceramide synthesis to reverse insulin resistance. Diabetes 59, 2351-2353 (2010).

134. J. R. Ussher et al., Inhibition of de novo ceramide synthesis reverses dietinduced insulin resistance and enhances whole-body oxygen consumption. Diabetes 59, 2453-2464 (2010).

135. M. A. Schroeder et al., The cycling of acetyl-coenzyme A through acetylcarnitine buffers cardiac substrate supply: a hyperpolarized $13 \mathrm{C}$ magnetic resonance study. Circulation. Cardiovascular imaging 5, 201209 (2012).

136. N. Siliprandi, F. Di Lisa, R. Menabo, Propionyl-L-carnitine: biochemical significance and possible role in cardiac metabolism. Cardiovascular drugs and therapy / sponsored by the International Society of Cardiovascular Pharmacotherapy 5 Suppl 1, 11-15 (1991).

137. H. Tapiero, G. Mathe, P. Couvreur, K. D. Tew, II. Glutamine and glutamate. Biomedicine \& pharmacotherapy = Biomedecine \& pharmacotherapie 56, 446-457 (2002).

138. G. Wu, Amino acids: metabolism, functions, and nutrition. Amino Acids 37, 1-17 (2009).

139. J. W. Ritchie et al., Mechanisms of glutamine transport in rat adipocytes and acute regulation by cell swelling. Cellular physiology and biochemistry : international journal of experimental cellular physiology, biochemistry, and pharmacology 11, 259-270 (2001).

140. S. Cheng et al., Metabolite profiling identifies pathways associated with metabolic risk in humans. Circulation 125, 2222-2231 (2012).

141. S. H. Adams, Emerging perspectives on essential amino acid metabolism in obesity and the insulin-resistant state. Advances in nutrition 2, 445-456 (2011).

142. C. B. Newgard et al., A branched-chain amino acid-related metabolic signature that differentiates obese and lean humans and contributes to insulin resistance. Cell Metab 9, 311-326 (2009).

143. M. A. Herman, P. She, O. D. Peroni, C. J. Lynch, B. B. Kahn, Adipose tissue branched chain amino acid (BCAA) metabolism modulates circulating BCAA levels. J Biol Chem 285, 11348-11356 (2010). 
144. P. She et al., Disruption of BCATm in mice leads to increased energy expenditure associated with the activation of a futile protein turnover cycle. Cell Metab 6, 181-194 (2007).

145. J. B. Regard, I. T. Sato, S. R. Coughlin, Anatomical profiling of G proteincoupled receptor expression. Cell 135, 561-571 (2008).

146. T. Cadoudal et al., Pyruvate dehydrogenase kinase 4: regulation by thiazolidinediones and implication in glyceroneogenesis in adipose tissue. Diabetes 57, 2272-2279 (2008).

147. O. E. Owen, S. C. Kalhan, R. W. Hanson, The key role of anaplerosis and cataplerosis for citric acid cycle function. J Biol Chem 277, 30409-30412 (2002).

148. B. K. Chacko et al., Methods for defining distinct bioenergetic profiles in platelets, lymphocytes, monocytes, and neutrophils, and the oxidative burst from human blood. Laboratory investigation; a journal of technical methods and pathology 93, 690-700 (2013).

149. S. Furukawa et al., Increased oxidative stress in obesity and its impact on metabolic syndrome. J Clin Invest 114, 1752-1761 (2004).

150. N. Matsuzawa-Nagata et al., Increased oxidative stress precedes the onset of high-fat diet-induced insulin resistance and obesity. Metabolism 57, 1071-1077 (2008).

151. S. Okada et al., Adipose tissue-specific dysregulation of angiotensinogen by oxidative stress in obesity. Metabolism 59, 1241-1251 (2010).

152. J. M. Curtis et al., Downregulation of adipose glutathione S-transferase A4 leads to increased protein carbonylation, oxidative stress, and mitochondrial dysfunction. Diabetes 59, 1132-1142 (2010).

153. E. K. Long, D. M. Olson, D. A. Bernlohr, High-fat diet induces changes in adipose tissue trans-4-oxo-2-nonenal and trans-4-hydroxy-2-nonenal levels in a depot-specific manner. Free Radic Biol Med 63, 390-398 (2013).

154. H. Asano et al., Diet-induced changes in Ucp1 expression in bovine adipose tissues. Gen Comp Endocrinol 184, 87-92 (2013).

155. J. X. Rong et al., Adipose mitochondrial biogenesis is suppressed in $\mathrm{db} / \mathrm{db}$ and high-fat diet-fed mice and improved by rosiglitazone. Diabetes 56, 1751-1760 (2007).

156. A. Ost et al., Attenuated mTOR signaling and enhanced autophagy in adipocytes from obese patients with type 2 diabetes. Molecular medicine 16, 235-246 (2010). 
157. J. Kovsan et al., Altered autophagy in human adipose tissues in obesity. The Journal of clinical endocrinology and metabolism 96, E268-277 (2011).

158. H. J. Jansen et al., Autophagy activity is up-regulated in adipose tissue of obese individuals and modulates proinflammatory cytokine expression. Endocrinology 153, 5866-5874 (2012).

159. G. Bjorkoy et al., Monitoring autophagic degradation of p62/SQSTM1. Methods in enzymology 452, 181-197 (2009).

160. D. J. Klionsky et al., Guidelines for the use and interpretation of assays for monitoring autophagy in higher eukaryotes. Autophagy 4, 151-175 (2008).

161. R. Singh et al., Autophagy regulates adipose mass and differentiation in mice. J Clin Invest 119, 3329-3339 (2009).

162. Y. Zhang et al., Adipose-specific deletion of autophagy-related gene 7 (atg7) in mice reveals a role in adipogenesis. Proc Natl Acad Sci U S A 106, 19860-19865 (2009).

163. M. Komatsu et al., Essential role for autophagy protein Atg7 in the maintenance of axonal homeostasis and the prevention of axonal degeneration. Proc Natl Acad Sci U S A 104, 14489-14494 (2007).

164. Q. J. Wang et al., Induction of autophagy in axonal dystrophy and degeneration. The Journal of neuroscience : the official journal of the Society for Neuroscience 26, 8057-8068 (2006).

165. E. S. Vincow et al., The PINK1-Parkin pathway promotes both mitophagy and selective respiratory chain turnover in vivo. Proc Natl Acad Sci U S A, (2013).

166. I. Murano et al., Dead adipocytes, detected as crown-like structures, are prevalent in visceral fat depots of genetically obese mice. J Lipid Res 49, 1562-1568 (2008).

167. S. Sam, T. Mazzone, Adipose tissue changes in obesity and the impact on metabolic function. Transl Res 164, 284-292 (2014).

168. N. DeJarnett et al., Acrolein exposure is associated with increased cardiovascular disease risk. J Am Heart Assoc 3, (2014).

169. E. Ciraci et al., Adult human circulating CD34- $\mathrm{Lin}^{-} \mathrm{CD} 45^{-} \mathrm{CD} 133^{-}$cells can differentiate into hematopoietic and endothelial cells. Blood 118, 21052115 (2011).

170. E. M. Van Craenenbroeck et al., Quantification of circulating CD34+/KDR+/CD45dim endothelial progenitor cells: analytical considerations. Int J Cardiol 167, 1688-1695 (2013). 
171. L. Zimmerlin, V. S. Donnenberg, J. P. Rubin, A. D. Donnenberg, Mesenchymal markers on human adipose stem/progenitor cells. Cytometry A 83, 134-140 (2013).

172. P. Trayhurn, B. Wang, I. S. Wood, Hypoxia in adipose tissue: a basis for the dysregulation of tissue function in obesity? Br J Nutr 100, 227-235 (2008).

173. J. Ratajczak et al., Adult murine bone marrow-derived very small embryonic-like stem cells differentiate into the hematopoietic lineage after coculture over OP9 stromal cells. Exp Hematol 39, 225-237 (2011).

174. H. Xu et al., Chronic inflammation in fat plays a crucial role in the development of obesity-related insulin resistance. J Clin Invest 112, 18211830 (2003).

175. M. J. Lee, Y. Wu, S. K. Fried, Adipose tissue remodeling in pathophysiology of obesity. Curr Opin Clin Nutr Metab Care 13, 371-376 (2010).

176. E. Okumachi et al., Comparative analysis of rat mesenchymal stem cells derived from slow and fast skeletal muscle in vitro. Int Orthop, (2014).

177. M. M. Levy et al., Osteoprogenitor cells of mature human skeletal muscle tissue: an in vitro study. Bone 29, 317-322 (2001).

178. M. F. Pittenger et al., Multilineage potential of adult human mesenchymal stem cells. Science 284, 143-147 (1999).

179. M. Kucia et al., Cells expressing early cardiac markers reside in the bone marrow and are mobilized into the peripheral blood after myocardial infarction. Circ Res 95, 1191-1199 (2004).

180. G. Li et al., Identification and characterization of chondrogenic progenitor cells in the fascia of postnatal skeletal muscle. J Mol Cell Biol 3, 369-377 (2011).

181. Z. Qu-Petersen et al., Identification of a novel population of muscle stem cells in mice: potential for muscle regeneration. J Cell Biol 157, 851-864 (2002).

182. A. Miranville et al., Improvement of postnatal neovascularization by human adipose tissue-derived stem cells. Circulation 110, 349-355 (2004).

183. P. A. Zuk et al., Human adipose tissue is a source of multipotent stem cells. Mol Biol Cell 13, 4279-4295 (2002).

184. B. E. Sansbury et al., Overexpression of endothelial nitric oxide synthase prevents diet-induced obesity and regulates adipocyte phenotype. Circ Res 111, 1176-1189 (2012). 
185. N. Hosogai et al., Adipose tissue hypoxia in obesity and its impact on adipocytokine dysregulation. Diabetes 56, 901-911 (2007).

186. J. Ye, Z. Gao, J. Yin, Q. He, Hypoxia is a potential risk factor for chronic inflammation and adiponectin reduction in adipose tissue of ob/ob and dietary obese mice. Am J Physiol Endocrinol Metab 293, E1118-1128 (2007).

187. M. E. Rausch, S. Weisberg, P. Vardhana, D. V. Tortoriello, Obesity in C57BL/6J mice is characterized by adipose tissue hypoxia and cytotoxic T-cell infiltration. Int J Obes (Lond) 32, 451-463 (2008).

188. E. M. Weijers et al., The influence of hypoxia and fibrinogen variants on the expansion and differentiation of adipose tissue-derived mesenchymal stem cells. Tissue Eng Part A 17, 2675-2685 (2011).

189. C. Fehrer et al., Reduced oxygen tension attenuates differentiation capacity of human mesenchymal stem cells and prolongs their lifespan. Aging Cell 6, 745-757 (2007).

190. Q. Lin, Y. J. Lee, Z. Yun, Differentiation arrest by hypoxia. J Biol Chem 281, 30678-30683 (2006).

191. E. Y. Lee et al., Hypoxia-enhanced wound-healing function of adiposederived stem cells: increase in stem cell proliferation and up-regulation of VEGF and bFGF. Wound Repair Regen 17, 540-547 (2009).

192. I. S. Wood, T. Stezhka, P. Trayhurn, Modulation of adipokine production, glucose uptake and lactate release in human adipocytes by small changes in oxygen tension. Pflugers Arch 462, 469-477 (2011).

193. E. E. Kershaw, J. S. Flier, Adipose tissue as an endocrine organ. J Clin Endocrinol Metab 89, 2548-2556 (2004).

194. Y. Okamoto, S. Kihara, T. Funahashi, Y. Matsuzawa, P. Libby, Adiponectin: a key adipocytokine in metabolic syndrome. Clin Sci (Lond) 110, 267-278 (2006).

195. Y. Kanda, T. Hinata, S. W. Kang, Y. Watanabe, Reactive oxygen species mediate adipocyte differentiation in mesenchymal stem cells. Life Sci $\mathbf{8 9}$, 250-258 (2011).

196. K. Schröder, K. Wandzioch, I. Helmcke, R. P. Brandes, Nox4 acts as a switch between differentiation and proliferation in preadipocytes. Arterioscler Thromb Vasc Biol 29, 239-245 (2009).

197. H. Yan et al., Nitric oxide promotes differentiation of rat white preadipocytes in culture. J Lipid Res 43, 2123-2129 (2002).

198. F. De Francesco et al., Human CD34/CD90 ASCs are capable of growing as sphere clusters, producing high levels of VEGF and forming capillaries. PLoS One 4, e6537 (2009). 
199. U. Forstermann, W. C. Sessa, Nitric oxide synthases: regulation and function. European heart journal 33, 829-837, 837a-837d (2012).

200. R. M. Cubbon, A. Rajwani, S. B. Wheatcroft, The impact of insulin resistance on endothelial function, progenitor cells and repair. Diab Vasc Dis Res 4, 103-111 (2007).

201. M. S. Kim et al., Regulation of diet-induced adipose tissue and systemic inflammation by salicylates and pioglitazone. PLoS One 8, e82847 (2013).

202. J. T. Crossno, S. M. Majka, T. Grazia, R. G. Gill, D. J. Klemm, Rosiglitazone promotes development of a novel adipocyte population from bone marrow-derived circulating progenitor cells. J Clin Invest 116, 32203228 (2006).

203. Y. J. Koh et al., Bone marrow-derived circulating progenitor cells fail to transdifferentiate into adipocytes in adult adipose tissues in mice. J Clin Invest 117, 3684-3695 (2007).

204. E. Rapizzi et al., Succinate dehydrogenase subunit B mutations modify human neuroblastoma cell metabolism and proliferation. Horm Cancer $\mathbf{5}$, 174-184 (2014).

205. Q. He et al., Regulation of HIF-1 \{alpha\} activity in adipose tissue by obesity-associated factors: adipogenesis, insulin, and hypoxia. $A m \mathrm{~J}$ Physiol Endocrinol Metab 300, E877-885 (2011).

206. W. He et al., Citric acid cycle intermediates as ligands for orphan Gprotein-coupled receptors. Nature 429, 188-193 (2004).

207. E. Nisoli et al., Effects of nitric oxide on proliferation and differentiation of rat brown adipocytes in primary cultures. Br J Pharmacol 125, 888-894 (1998).

208. E. Nisoli et al., Mitochondrial biogenesis in mammals: the role of endogenous nitric oxide. Science 299, 896-899 (2003).

209. T. T. Tran, C. R. Kahn, Transplantation of adipose tissue and stem cells: role in metabolism and disease. Nat Rev Endocrinol 6, 195-213 (2010).

210. H. Green, O. Kehinde, Formation of normally differentiated subcutaneous fat pads by an established preadipose cell line. J Cell Physiol 101, 169171 (1979).

211. C. Fischbach et al., Generation of mature fat pads in vitro and in vivo utilizing 3-D long-term culture of 3T3-L1 preadipocytes. Exp Cell Res 300, 54-64 (2004).

212. R. L. Van, D. A. Roncari, Complete differentiation in vivo of implanted cultured adipocyte precursors from adult rats. Cell Tissue Res 225, 557566 (1982). 
213. H. Mizuno et al., In vivo adipose tissue regeneration by adipose-derived stromal cells isolated from GFP transgenic mice. Cells Tissues Organs 187, 177-185 (2008).

214. Y. S. Kim et al., Hypoxia/Reoxygenation-Preconditioned Human Bone Marrow-Derived Mesenchymal Stromal Cells Rescue Ischemic Rat Cortical Neurons by Enhancing Trophic Factor Release. Mol Neurobiol, (2014). 


\section{CURRICULUM VITAE}

NAME: $\quad$ Candice ReShay Holden

ADDRESS: $\quad$ University of Louisville

Diabetes and Obesity Center

580 South Preston Street, Room 411

(502) 852-4099

Louisville, KY 40202

DOB: $\quad$ Huntsville, Alabama - January 17, 1988

\section{EDUCATION}

\& TRAINING: B.S., Molecular Biology and Microbiology University of Central Florida

2006-2010

M.S., Physiology and Biophysics

University of Louisville

2010-2013

Ph.D., Physiology and Biophysics

University of Louisville

2010-2014

AWARDS: $\quad$ NIH/NHLBI supported Pre-doctoral Supplement Grant (P01HL078825)

Title: Protection of Ischemic Myocardium

August 2013- May 2015

Pre-doctoral Graduate Fellowship

University of Louisville

August 2010 - December 2014

Dean's List for the School of Medicine University of Central Florida 
Pegasus Merit Scholarship University of Central Florida August 2006-May 2008

100\% Florida Bright Futures Scholarship Award University of Central Florida August 2006 - May 2010

PROFESSIONAL SOCIETIES:

American Heart Association (AHA)

- $\quad$ Council on Nutrition, Physical Activity, and Metabolism

- Council on Lifestyle and Cardiometabolic Health

American Physiological Society (APS)

- $\quad$ Cell \& Molecular Physiology Section

- $\quad$ Endocrinology \& Metabolism Section

American Association for the Advancement of Science (AAAS)

Kentucky Academy of Science (KAS)

National Black Graduate Student Association (NBGSA)

Sisters of the Academy (SOTA)

RELATED EXPERIENCE AND ORGANIZATIONS:

Sisters of the Academy (SOTA)

Member

2013- Present

National Black Graduate Student Association- Southeast region (NBGSA) Member

2013- Present

Black Biomedical Graduate Student Organization (BBGSO)

University of Louisville, Louisville, KY

Vice President

2012- Present

U.S. Wellness

Central Kentucky and Southern Indiana

Health Screener

2012- Present

Department of Physiology and Biophysics 
University of Louisville

Course Facilitator for Integrated Systemic Physiology (PHZB 609)

Spring 2013

Science Olympiad

DuPont Manual High School, Louisville, KY

Supervisor/Judge for "Disease Detectives" section for middle and high school students

Spring 2012

American Physiological Society PhUn Week, Louisville, KY

Volunteer

2011

Integrated Programs in Biomedical Sciences (IPIBS)

University of Louisville, Louisville, KY

Graduate Fellowship Recipient ～(August 2010- August 2012)

Recruiter (2013)

Student panel representative (2012-2014)

Student Osteopathic Medical Association (Pre-SOMA)

University of Central Florida, Orlando, FL

Chapter Co-founder and Vice President

2008- 2010

Delta Epsilon Mu

University of Central Florida, Orlando, FL

Education Chair and Secretary

2008- 2010

Burnett Honors College

University of Central Florida

Team Leader

2007- 2008

Junior Achievement of Orlando (J.A.)

Orlando, FL

Trainer and Educator

2006- 2008 


\section{LABORATORY/TECHNICAL SKILLS}

Animal Techniques:

Stem Cell Research:

Bone Marrow Cell Isolation

Bone marrow transplants (some experience)

Anesthetization

Stromal Vascular Fraction Isolation

Organ harvest

Metabolic cage analysis

Adiposity assessment via Dual-energy X-ray absorptiometry

Injections (intraperitoneal, subcutaneous)

Glucose and Insulin tolerance tests

Spinal Cord Injury Research:

Anesthetization

Contusion injuries

Suturing

Perfusions

Molecular Biology Techniques:

Multi-color Flow Cytometry (BD LSRII and Accuri C6)

Light Microscopy

Fluorescent Microscopy

Confocal Microscopy (some experience)

Cell culture:

Primary cell isolations: cardiac stem cells, endothelial progenitor cells, mesenchymal stem cells, stromal vascular fraction, bone marrow-derived macrophages, and bone marrow-derived stem cells

Colony-Forming unit assays

Adipocyte differentiation of human and murine mesenchymal stem cells

Proliferation assays

Tube-forming assay

Cell Sorting (FACS): mesenchymal stem cells, endothelial progenitor cells, and immune cells

Aseptic techniques

Human Tissue Specimens:

Blood tube processing

Stem cell isolation from adipose tissue and blood

White blood cell isolation from adipose tissue and blood

Tissue storage and preservation 
Data Analysis:

FlowJo

BD FACSDiva Software

SPSS

Prism- GraphPad

Microsoft Office: Access, Excel, PowerPoint Presentations, and Word

\section{PUBLICATIONS:}

${ }^{*}$ Cummins TD, *Holden CR, *Sansbury BE, Gibb AA, Shah J, Zafar N, Tang Y, Hellmann J, Rai SN, Spite M, Bhatnagar A, and Hill BG.

Metabolic remodeling of white adipose tissue in obesity. Am J Physiol Endocrinol Metab 307:E262-E277, 2014.

Salabei JK, Lorkiewicz PK, Holden CR, Li Q, Hong KU, Bolli R, Bhatnagar A, and Hill BG. Glutamine regulates cardiac stem cell metabolism and proliferation. Submitted for publication in December 2014.

Sansbury BE, Cummins TD, Tang Y, Hellmann J, Holden CR, Harbeson MA, Chen Y, Patel RP, Spite M, Bhatnagar A, and Hill BG.

Overexpression of endothelial nitric oxide synthase prevents diet-induced obesity and regulates adipocyte phenotype. Circ Res. 111(9):1176-89, 2012.

\section{ABSTRACTS AND POSTER PRESENTATIONS:}

Holden CR, Wysoczynski M, Sansbury BE, Hellmann JL, Zafar N, Gibb AA, Bhatnagar A, Hill BG. Nutrient excess promotes accumulation of bone marrow derived progenitor cells in adipose tissue. Poster presentation, 2014 Experimental Biology: San Diego, CA.

Holden CR, Wysoczynski M, Sansbury BE, Hellmann JL, Zafar N, Gibb AA, Bhatnagar A, Hill BG. Dynamics Of Adipocyte And Endothelial Progenitor Cell Pools In Expanding Adipose Tissue. Poster presentation at 2014 American Heart Association Scientific Sessions: Chicago, IL.

Salabei JK, Lorkiewicz PK, Holden CR, Harbeson MA, Li Q, Bolli R, Bhatnagar A, Hill BG. Glutamine regulates cardiac stem cell metabolism and competency. Poster presentation at 2014 American Heart Association Scientific Sessions: Chicago, IL.

Zafar N, Krishnasamy SS, Shah J, McCraken J, Holden CR, DeJarnett NK, Abplanalp WT, Hill BG, Conklin DJ, O'Toole T, Rai SN, Bhatnagar A. 
Depletion of circulating CD34+/KDR+ cells in Type 2 Diabetes is associated with glycemic control. Poster presentation at 2014 American Heart Association Scientific Sessions: Chicago, IL.

Cummins TD, Guo Y, Du J, Book M, Zhu X, Wu W, McCracken JP, Salabei J, Zafar N, Haberzettl P, Holden CR, Gibb A, Jones SP, Li Q, Bolli R, Bhatnagar A, Hill BG. Type 2 diabetes decreases cardiac stem cell mitochondrial metabolism and prevents myocardial repair. 2013

Holden CR, McCracken J, Sansbury BE, Hellmann J, Cummins TD, Bhatnagar A, Hill BG. Regulation of Adipose Tissue Stem Cells by Inflammation. Poster Presentation at Research! Louisville, 2012.

Cummins TD, Sansbury BE, Holden CR, Bhatnagar A, Hill BG. Metabolic remodeling of white adipose tissue in obesity. Society for Free Radical Biology and Medicine (SFRBM), 2012. 